\title{
Reversible Dioxygen Scavenging from Air by a Copper Complex
}

\author{
Kurtis M. Carsch, ${ }^{\dagger *}$ Andrei Iliescu, ${ }^{\dagger *}$ Ryan D. McGillicuddy, ${ }^{\dagger}$ Jarad A. Mason, ${ }^{\dagger}$ \\ Theodore A. Betley ${ }^{\dagger}$ \\ ${ }^{\dagger}$ Department of Chemistry and Chemical Biology \\ Harvard University \\ 12 Oxford Street, Cambridge, MA 02138 \\ *These authors contributed equally.
}

Corresponding Author: betley@chemistry.harvard.edu 


$\begin{array}{ll}\text { Table of Contents } & \text { S2 }\end{array}$

Materials and Methods $\quad$ S4

General Considerations $\quad$ S4

Characterization and Physical Measurements $\quad$ S5

$\begin{array}{ll}\text { Metal Complex Syntheses } & \text { S6 }\end{array}$

( $\left.{ }^{\text {EMind }} \mathrm{L}\right) \mathrm{Cu}\left(\mathrm{O}_{2}\right)(\mathbf{2})$ Synthesis $\quad$ S6

Figure S1. ${ }^{1} \mathrm{H}$ NMR Spectrum of $\left({ }^{\mathrm{EMind}} \mathrm{L}\right) \mathrm{Cu}\left(\mathrm{O}_{2}\right)(\mathbf{2}) \quad$ S7

$\begin{array}{ll}\text { Figure S2. }{ }^{13} \mathrm{C} \text { NMR Spectrum of }\left({ }^{\mathrm{EMind}} \mathrm{L}\right) \mathrm{Cu}\left(\mathrm{O}_{2}\right)(\mathbf{2}) & \text { S7 }\end{array}$

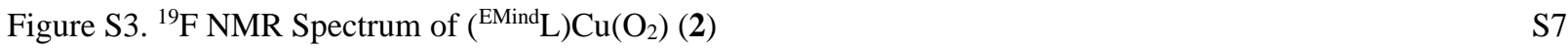

Figure S4. High Resolution Mass Spectrometry (HRMS) of $\left({ }^{\mathrm{EMind}} \mathrm{L}\right) \mathrm{Cu}\left(\mathrm{O}_{2}\right)(\mathbf{2}) \quad$ S8

Figure S5. Cyclic Voltammogram of $\left({ }^{\mathrm{EMind}} \mathrm{L}\right) \mathrm{Cu}\left(\mathrm{O}_{2}\right)(\mathbf{2}) \quad$ S9

$\begin{array}{lr}\left({ }^{\text {EMind }} \mathrm{L}\right) \mathrm{Cu} \text { (toluene) (3) Synthesis } & \mathrm{S} 10\end{array}$

Figure S6. ${ }^{1} \mathrm{H}$ NMR Spectrum of $\left({ }^{\mathrm{EMind}} \mathrm{L}\right) \mathrm{Cu}$ (toluene) (3) $\quad$ S11

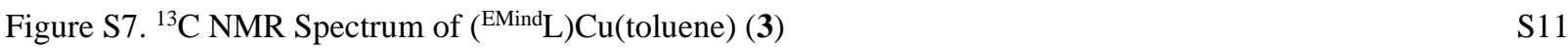

Figure S8. ${ }^{19} \mathrm{~F}$ NMR Spectrum of ( $\left.{ }^{\text {EMind }} \mathrm{L}\right) \mathrm{Cu}$ (toluene) $(\mathbf{3}) \quad$ S11

(EMind $\mathrm{L}) \mathrm{Cu}\left(\mathrm{CH}_{2} \mathrm{Cl}_{2}\right)$ (4) Synthesis $\quad \mathrm{S} 12$

Figure S9. ${ }^{1} \mathrm{H}$ NMR Spectrum of $\left({ }^{\mathrm{EMind}} \mathrm{L}\right) \mathrm{Cu}\left(\mathrm{CH}_{2} \mathrm{Cl}_{2}\right)(\mathbf{4}) \quad \mathrm{S} 13$

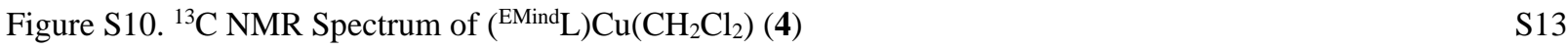

Figure S11. ${ }^{19} \mathrm{~F}$ NMR Spectrum of $\left({ }^{\mathrm{EMind}} \mathrm{L}\right) \mathrm{Cu}\left(\mathrm{CH}_{2} \mathrm{Cl}_{2}\right)(\mathbf{4}) \quad \mathrm{S} 13$

( $\left.{ }^{\text {EMind }} \mathrm{L}\right) \mathrm{Cu}\left(\mathrm{NH}_{2} \mathrm{NH}_{2}\right)(\mathbf{5})$ Synthesis $\quad \mathrm{S} 14$

Figure S12. ${ }^{1} \mathrm{H}$ NMR Spectrum of $\left({ }^{\mathrm{EMind}} \mathrm{L}\right) \mathrm{Cu}\left(\mathrm{NH}_{2} \mathrm{NH}_{2}\right)(\mathbf{5}) \quad \mathrm{S} 15$

Figure S13. ${ }^{13} \mathrm{C}$ NMR Spectrum of $\left({ }^{\mathrm{EMind}} \mathrm{L}\right) \mathrm{Cu}\left(\mathrm{NH}_{2} \mathrm{NH}_{2}\right)(\mathbf{5}) \quad \mathrm{S} 15$

Figure S14. ${ }^{19} \mathrm{~F}$ NMR Spectrum of $\left({ }^{\mathrm{EMind}} \mathrm{L}\right) \mathrm{Cu}\left(\mathrm{NH}_{2} \mathrm{NH}_{2}\right)(\mathbf{5}) \quad \mathrm{S} 15$

$\begin{array}{ll}\left({ }^{\mathrm{EMind}} \mathrm{L}\right) \mathrm{CuI}(\mathbf{6}) \text { Synthesis } & \text { S16 }\end{array}$

$\begin{array}{ll}\text { Figure S15. }{ }^{1} \mathrm{H} \text { NMR Spectrum of }\left({ }^{\mathrm{EMind}} \mathrm{L}\right) \mathrm{CuI}(\mathbf{6}) & \mathrm{S} 17\end{array}$

$\begin{array}{ll}\text { Figure S16. }{ }^{19} \mathrm{~F} \text { NMR Spectrum of }\left({ }^{\mathrm{EMind}} \mathrm{L}\right) \mathrm{CuI}(\mathbf{6}) & \mathrm{S} 17\end{array}$

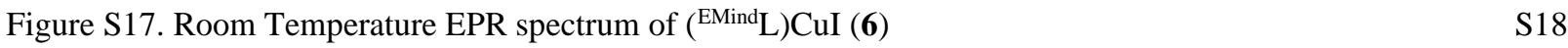

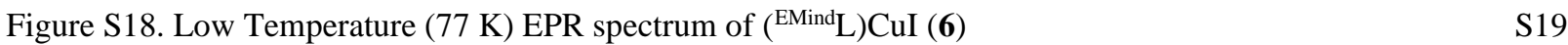

Figure S19. Cyclic Voltammogram of ( ${ }^{\text {EMind }}$ L)CuI (6) $\quad$ S20

$\begin{array}{ll}\left({ }^{\text {EMind }} \mathrm{L}\right) \mathrm{Cu}\left(\mathrm{H}_{2} \mathrm{O}_{2}\right)(7) \text { Synthesis } & \text { S21 }\end{array}$

Figure S20. ${ }^{1} \mathrm{H}$ NMR Spectrum of $\left({ }^{\mathrm{EMind}} \mathrm{L}\right) \mathrm{Cu}\left(\mathrm{H}_{2} \mathrm{O}_{2}\right)(7) \quad$ S22

Figure S21. ${ }^{19} \mathrm{~F}$ NMR Spectrum of $\left({ }^{\mathrm{EMind}} \mathrm{L}\right) \mathrm{Cu}\left(\mathrm{H}_{2} \mathrm{O}_{2}\right)(7) \quad$ S22

Figure S22. Independent Synthesis and Comparison of ( $\left.{ }^{\mathrm{EMind}} \mathrm{L}\right) \mathrm{Cu}\left(\mathrm{H}_{2} \mathrm{O}_{2}\right)(\mathbf{7}) \quad$ S23

$\begin{array}{ll}\text { Supporting Spectroscopic Characterization } & \text { S24 }\end{array}$

Figure S23. Visual Comparison between ( $\left.{ }^{\mathrm{EMind}} \mathrm{L}\right) \mathrm{Cu}\left(\mathrm{N}_{2}\right)(\mathbf{1})$ and $\left({ }^{\mathrm{EMind}} \mathrm{L}\right) \mathrm{Cu}\left(\mathrm{O}_{2}\right)(\mathbf{2}) \quad$ S24

Figure S24. ${ }^{1} \mathrm{H}$ NMR Spectra of $\left({ }^{\mathrm{Ar}} \mathrm{L}\right) \mathrm{Cu}$ and Air Exposure $\quad$ S24

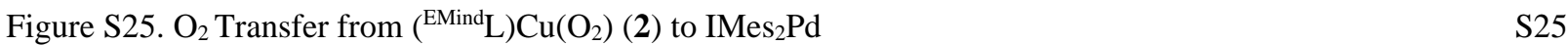

Solvent Cycling Supporting NMR Spectra $\quad$ S26

Figure S26. ${ }^{1} \mathrm{H}$ NMR Spectrum of $\left({ }^{\mathrm{EMind}} \mathrm{L}\right) \mathrm{Cu}$ (toluene) $(3)$ from $\left({ }^{\mathrm{EMind}} \mathrm{L}\right) \mathrm{Cu}\left(\mathrm{O}_{2}\right)(\mathbf{2}) \quad$ S27

Figure S27. ${ }^{19} \mathrm{~F}$ NMR Spectrum of $\left({ }^{\mathrm{EMind}} \mathrm{L}\right) \mathrm{Cu}$ (toluene) $(\mathbf{3})$ from $\left({ }^{\mathrm{EMind}} \mathrm{L}\right) \mathrm{Cu}\left(\mathrm{O}_{2}\right)(\mathbf{2}) \quad$ S27

Figure S28. ${ }^{1} \mathrm{H}$ NMR Spectrum of $\left({ }^{\mathrm{EMind}} \mathrm{L}\right) \mathrm{Cu}\left(\mathrm{O}_{2}\right)(\mathbf{2})$ from $\left({ }^{\mathrm{EMind}} \mathrm{L}\right) \mathrm{Cu}$ (toluene) $(\mathbf{3}) \quad$ S28

Figure S29. ${ }^{19} \mathrm{~F}$ NMR Spectrum of $\left({ }^{\mathrm{EMind}} \mathrm{L}\right) \mathrm{Cu}\left(\mathrm{O}_{2}\right)(\mathbf{2})$ from $\left({ }^{\mathrm{EMind}} \mathrm{L}\right) \mathrm{Cu}$ (toluene) $(\mathbf{3}) \quad$ S28

Figure S30. ${ }^{1} \mathrm{H}$ NMR Spectrum of ( $\left.{ }^{\mathrm{EMind}} \mathrm{L}\right) \mathrm{Cu}\left(\mathrm{CH}_{2} \mathrm{Cl}_{2}\right)(\mathbf{4})$ from $\left({ }^{\mathrm{EMind}} \mathrm{L}\right) \mathrm{Cu}\left(\mathrm{O}_{2}\right)(\mathbf{2}) \quad$ S29

Figure S31. ${ }^{19} \mathrm{~F}$ NMR Spectrum of $\left({ }^{\mathrm{EMind}} \mathrm{L}\right) \mathrm{Cu}\left(\mathrm{CH}_{2} \mathrm{Cl}_{2}\right)(\mathbf{4})$ from $\left({ }^{\mathrm{EMind}} \mathrm{L}\right) \mathrm{Cu}\left(\mathrm{O}_{2}\right)(\mathbf{2}) \quad$ S29

Figure S32. ${ }^{1} \mathrm{H}$ NMR Spectrum of $\left({ }^{\mathrm{EMind}} \mathrm{L}\right) \mathrm{Cu}\left(\mathrm{O}_{2}\right)(\mathbf{2})$ from $\left({ }^{\mathrm{EMind}} \mathrm{L}\right) \mathrm{Cu}\left(\mathrm{CH}_{2} \mathrm{Cl}_{2}\right)(\mathbf{4}) \quad$ S30

Figure S33. ${ }^{19} \mathrm{~F}$ NMR Spectrum of $\left({ }^{\mathrm{EMind}} \mathrm{L}\right) \mathrm{Cu}\left(\mathrm{O}_{2}\right)(\mathbf{2})$ from $\left({ }^{\mathrm{EMind}} \mathrm{L}\right) \mathrm{Cu}\left(\mathrm{CH}_{2} \mathrm{Cl}_{2}\right)(\mathbf{4}) \quad$ S30

Table S1. Additional Ligand Displacement Observations $\quad$ S31 
Redox Cycling Supporting NMR Spectra

Figure S34. ${ }^{1} \mathrm{H}$ NMR Spectra of reformation of $\left({ }^{\mathrm{EMind}} \mathrm{L}\right) \mathrm{Cu}\left(\mathrm{O}_{2}\right)(\mathbf{2})$ from $\left({ }^{\mathrm{EMind}} \mathrm{L}\right) \mathrm{CuI}(\mathbf{6}) \quad$ S33

Figure S35. ${ }^{19} \mathrm{~F}$ NMR Spectra of reformation of $\left({ }^{\mathrm{EMind}} \mathrm{L}\right) \mathrm{Cu}\left(\mathrm{O}_{2}\right)(\mathbf{2})$ from $\left({ }^{\mathrm{EMind}} \mathrm{L}\right) \mathrm{CuI}(\mathbf{6}) \quad$ S33

Thermal Cycling Supporting NMR Spectra $\quad$ S34

Figure S36-S37. TGA Cycling Studies $\quad$ S34

Figure S38. ${ }^{1} \mathrm{H}$ NMR Spectrum of post-TGA $\left({ }^{\mathrm{EMind}} \mathrm{L}\right) \mathrm{Cu}\left(\mathrm{O}_{2}\right)(\mathbf{2}) \quad$ S35

Figure S39. ${ }^{19} \mathrm{~F}$ NMR Spectrum of post-TGA $\left({ }^{\mathrm{EMind}} \mathrm{L}\right) \mathrm{Cu}\left(\mathrm{O}_{2}\right)(\mathbf{2}) \quad$ S35

Figure S40. ${ }^{1} \mathrm{H}$ NMR Spectrum of ( $\left.{ }^{\text {EMind }} \mathrm{L}\right) \mathrm{Cu}\left(\mathrm{O}_{2}\right)(\mathbf{2})$ under $\mathrm{N}_{2}$ Atmosphere $\quad$ S36

Figure S41. ${ }^{19} \mathrm{~F}$ NMR Spectrum of $\left({ }^{\text {EMind }} \mathrm{L}\right) \mathrm{Cu}\left(\mathrm{O}_{2}\right)(\mathbf{2})$ under $\mathrm{N}_{2}$ Atmosphere $\quad$ S36

Figure S42. ${ }^{1} \mathrm{H}$ NMR Spectrum of $\left({ }^{\mathrm{EMind}} \mathrm{L}\right) \mathrm{Cu}\left(\mathrm{O}_{2}\right)(\mathbf{2})$ Following Solid-state Thermolysis $\quad$ S37

$\begin{array}{ll}\text { Figure S43. TGA Temperature Sweep under Ar } & \text { S37 }\end{array}$

$\begin{array}{ll}\text { Single-Crystal-to-Single-Crystal Transformations } & \text { S38 }\end{array}$

Figure S44 to S48. Supporting Characterization of Crystalline Transformations $\quad$ S38

Hydrazine Oxidation NMR Spectra $\quad$ S43

Figure S49. Post-catalytic ${ }^{1}$ H NMR Spectrum of 1,2-diphenylhydrazine Oxidation $\quad$ S44

Figure S50. ${ }^{1} \mathrm{H}$ NMR Spectrum of 1,2-diphenylhydrazine Oxidation with Comparison to Standards $\quad$ S44

Figure S51. Post-catalytic ${ }^{1} \mathrm{H}$ NMR Spectrum of 1,2-diphenylhydrazine Oxidation in Hexanes $\quad$ S44

Figure S52. Post-catalytic ${ }^{1} \mathrm{H}$ NMR Spectrum of 1,2-diphenylhydrazine Oxidation in Absence of $2 \quad$ S45

Figure S53. Post-catalytic ${ }^{1} \mathrm{H}$ NMR Spectrum of Hydrogen Peroxide Formation $\quad$ S45

Figure S54. Geometry Optimization of ( $\left.{ }^{\mathrm{EMind}} \mathrm{L}\right) \mathrm{Cu}\left(\mathrm{H}_{2} \mathrm{O}_{2}\right)(7) \quad S 46$

$\begin{array}{ll}\text { Crystallography Details } & \text { S50 }\end{array}$

Table S2. Single Crystal X-ray Diffraction Metrics $\quad$ S50

XRD Refinement Details $\quad$ S52

Table S3. Pertinent Bond Metrics of $\left({ }^{\mathrm{EMind}} \mathrm{L}\right) \mathrm{Cu}\left(\eta^{2}-\mathrm{C}_{6} \mathrm{H}_{6}\right)(\mathbf{1}$-benzene) at $100 \mathrm{~K} \quad$ S55

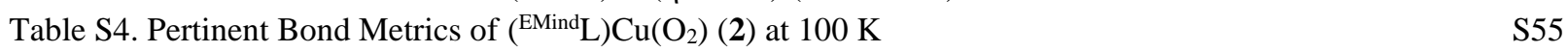

Table S5. Pertinent Bond Metrics of ( $\left.{ }^{\mathrm{EMind}} \mathrm{L}\right) \mathrm{Cu}\left(\mathrm{CH}_{2} \mathrm{Cl}_{2}\right)(\mathbf{4}) \quad$ S55

Table S6. Pertinent Bond Metrics of ( $\left.{ }^{\mathrm{EMind}} \mathrm{L}\right) \mathrm{Cu}\left(\mathrm{NH}_{2} \mathrm{NH}_{2}\right)(\mathbf{5}) \quad$ S55

Table S7. Pertinent Bond Metrics of ( $\left.{ }^{\mathrm{EMind}} \mathrm{L}\right) \mathrm{CuI}(\mathbf{6}) \quad$ S55

Figure S55. Solid-state Structure of $\left({ }^{\mathrm{EMind}} \mathrm{L}\right) \mathrm{Cu}\left(\eta^{2}-\mathrm{C}_{6} \mathrm{H}_{6}\right)$ (1-benzene) $\quad$ S56

$\begin{array}{ll}\text { Figure S56. Solid-state Structure of }\left({ }^{\mathrm{EMind}} \mathrm{L}\right) \mathrm{Cu}\left(\mathrm{O}_{2}\right)(2) \text { at } 100 \mathrm{~K} & \text { S57 }\end{array}$

$\begin{array}{ll}\text { Figure S57. Solid-state Structure of }\left({ }^{\mathrm{EMind}} \mathrm{L}\right) \mathrm{Cu}\left(\mathrm{CH}_{2} \mathrm{Cl}_{2}\right)(\mathbf{4}) & \text { S58 }\end{array}$

Figure S58. Solid-state Structure of $\left({ }^{\mathrm{EMind}} \mathrm{L}\right) \mathrm{Cu}\left(\mathrm{NH}_{2} \mathrm{NH}_{2}\right)(\mathbf{5}) \quad$ S59

Figure S59. Solid-state Structure of ( ${ }^{\text {EMind }}$ L)CuI (6) $\quad$ S60

$\begin{array}{ll}\text { References } & \text { S61 }\end{array}$

$\begin{array}{ll}\text { CheckCIF Output Files } & \text { S62 }\end{array}$ 


\section{Materials and Methods.}

General Considerations. All manipulations were carried out under ambient air in a wellventilated fume hood, unless specified otherwise. For air-sensitive manipulations, an MBraun inert atmosphere drybox containing either dinitrogen or argon was employed to exclude water and dioxygen. For manipulations in the drybox, all glassware was oven dried at $150^{\circ} \mathrm{C}$ for a minimum of $12 \mathrm{~h}$ and cooled in an evacuated antechamber for a minimum of 15 minutes. Benzene, diethyl ether, dichloromethane (DCM), acetonitrile $(\mathrm{MeCN})$, hexanes, pentane, toluene, and tetrahydrofuran (THF) were dried over $4 \AA$ molecular sieves (Strem) prior to use in the drybox. When applicable, solvents were tested with a deep violet solution of sodium benzophenone ketyl in tetrahydrofuran (prepared by stirring $10 \mathrm{mg}$ benzophenone in $10 \mathrm{~mL}$ THF with excess metallic sodium for $12 \mathrm{~h}$ ) to confirm effective oxygen and moisture removal. Chloroform- $d_{1}$ was purchased from Cambridge Isotope Labs and stored over anhydrous potassium carbonate for employment under air. Benzene- $d_{6}$ and tetrahydrofuran- $d_{8}$ were purchased from Cambridge Isotope Labs, degassed, and stored over $4 \AA$ molecular sieves prior to use in the drybox. Reagents potassium graphite $\left(\mathrm{KC}_{8}\right)^{1},\left(\mathrm{PPh}_{3} \mathrm{O}\right)_{2}\left(\mathrm{H}_{2} \mathrm{O}_{2}\right)^{2}$, (IMes) $)_{2} \mathrm{Pd}^{3}$, and $(\mathrm{IMes})_{2} \mathrm{Pd}\left(\mathrm{O}_{2}\right)^{3}$ were synthesized according to literature protocols. Elemental iodine was sublimed prior to use and stored in a freezer with exclusion of light. Reagent 1,2-diphenylhydrazine was crystallized from slow cooling of warm diethyl ether under dinitrogen to ensure removal of trace azoarene. Hydrazine $\left(\mathrm{NH}_{2} \mathrm{NH}_{2}\right)$, hydrazine monohydrate $\left(\mathrm{NH}_{2} \mathrm{NH}_{2} \bullet \mathrm{H}_{2} \mathrm{O}\right)$, and $30 \%$ aqueous hydrogen peroxide $\left(\mathrm{H}_{2} \mathrm{O}_{2}\right)$ were purchased from Sigma-Aldrich and used as received. ( $\left.{ }^{\mathrm{EMind}} \mathrm{L}\right) \mathrm{Cu}\left(\mathrm{N}_{2}\right)(\mathbf{1})$ was prepared according to literature procedures ${ }^{4}$. Celite ${ }^{\circledR} 545$ (J. T. Baker) was dried in a Schlenk flask for $24 \mathrm{~h}$ under dynamic vacuum while heating to at least $150{ }^{\circ} \mathrm{C}$ prior to use in a drybox. Activated alumina was dried in a Schlenk flask for $48 \mathrm{~h}$ under dynamic vacuum at $200{ }^{\circ} \mathrm{C}$ prior to use in a drybox. Silica gel 32-63 $\mu$ (AIC, Framingham, MA) was used as received. 


\section{Characterization and Physical Measurements.}

NMR Spectroscopy. ${ }^{1} \mathrm{H},{ }^{13} \mathrm{C}\left\{{ }^{1} \mathrm{H}\right\},{ }^{31} \mathrm{P}$, and ${ }^{19} \mathrm{~F}$ NMR spectra were recorded on Varian Unity/Inova 400, 500, or $600 \mathrm{MHz}$ spectrometers. ${ }^{1} \mathrm{H}$ and ${ }^{13} \mathrm{C}\left\{{ }^{1} \mathrm{H}\right\}$ NMR chemical shifts are reported relative to $\mathrm{SiMe}_{4}$ using the chemical shift of residual solvent peaks as reference. ${ }^{19} \mathrm{~F} \mathrm{NMR}$ chemical shifts are reported relative to an external standard of boron $\mathrm{BF}_{3}\left(\mathrm{OEt}_{2}\right)(\delta-153.00 \mathrm{ppm})$. ${ }^{31} \mathrm{P}$ NMR chemical shifts are referenced to an external standard of $85 \% \mathrm{H}_{3} \mathrm{PO}_{4}(\delta 0.00 \mathrm{ppm})$. Multiplicity assignments are abbreviated as follows: $\mathrm{s}=$ singlet, $\mathrm{d}=$ doublet, $\mathrm{t}=$ triplet, $\mathrm{q}=$ quartet, $\mathrm{m}=$ multiplet, $\mathrm{br}=$ broad, $\mathrm{v}$. br $=$ very broad .

Resonance Raman Spectroscopy. Resonance Raman measurements were collected at the Center for Nanoscale Systems (CNS) at Harvard University, a member of the National Nanotechnology Coordinated Infrastructure Network (NNCI). Raman spectra were recorded using a Horiba LabRam HR Evolution micro-Raman spectrometer featuring $<1 \mathrm{~cm}^{-1}$ spectral resolution and sub- $\mu \mathrm{m}$ spatial resolution for confocal imaging. Measurements were conducted using a 532 $\mathrm{nm}$ excitation laser $(0.028 \mathrm{~mW})$ at room temperature on solid samples.

Optical Spectroscopy. UV/Visible/NIR spectra were recorded on a Varian Cary 50 UV/Vis spectrometer using air-free quartz cuvettes $(0.10 \mathrm{~mm}$ path length) and a scan rate of $600 \mathrm{~nm} / \mathrm{min}$. Samples were prepared using serial dilution with volumetric glassware to obtain accurate concentrations. Extinction coefficients were determined from a minimum of three concentrations per sample and were calculated by a linear regression fit of the absorbance vs. concentration data.

Electron Paramagnetic Resonance Spectroscopy. EPR spectra were obtained on a Bruker EleXsys E-500 CW-EPR spectrometer. Spectra containing ca. 5-10 mg sample were measured as frozen toluene glasses, frozen 2-methyltetrahydrofuran glasses, and/or in solution at a microwave power of 0.6325-2 $\mathrm{mW}$. Effective g-values, g-strain $(\sigma)$, and hyperfine coupling constants (A) were obtained from spectral simulations of $S=1 / 2$ systems using the program Easyspin. ${ }^{5}$

Electrochemistry. Cyclic voltammetry and differential pulse voltammetry measurements were performed with a CHI660d potentiostat using a three-electrode cell with a glassy carbon working electrode, a platinum wire as the counter electrode, and an $\mathrm{Ag} / \mathrm{AgNO} 3$ reference electrode. All of the potentials are referenced to the $\left[\mathrm{Cp}_{2} \mathrm{Fe}\right]^{+/ 0}$ couple. Saturated tetrabutylammonium hexafluorophosphate $\left(\mathrm{TBAPF}_{6}\right)$ solutions of $0.2 \mathrm{M}$ in tetrahydrofuran were prepared before each experiment. All measurements were conducted under a dinitrogen atmosphere. No background reaction upon addition of excess $\mathrm{TBAPF}_{6}$ in tetrahydrofuran was observed for all complexes.

Combustion Analysis. Elemental analysis (\%CHN) was conducted at Harvard University on a PerkinElmer 2400 Series II CHNS/O Analyzer using bulk recrystallized compounds. Samples were prepared in a dinitrogen-filled drybox, which prevented satisfactory characterization on several complexes due to facile displacement with $\mathrm{N}_{2}$. In several cases, satisfactory elemental analyses were obtained by considering the presence of solvent molecules intercalated within the unit cell as ascertained by single-crystal X-ray diffraction and ${ }^{1} \mathrm{H}$ NMR spectroscopy. 
Metal Complex Syntheses.

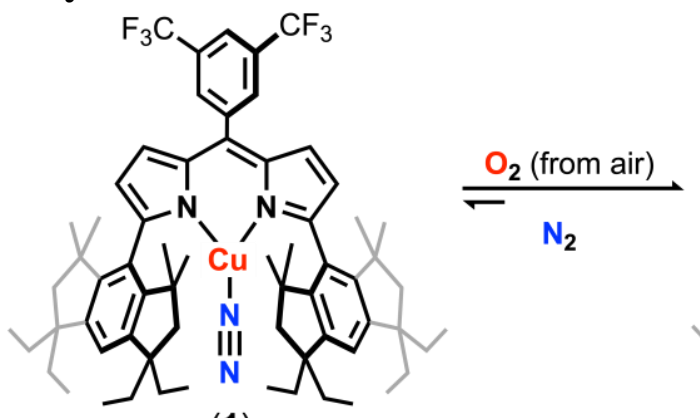

(1)

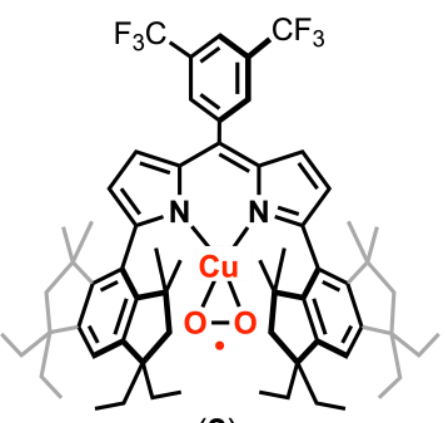

(2)

( $\left.{ }^{\mathbf{E M i n d}} \mathbf{L}\right) \mathbf{C u}\left(\mathbf{O}_{2}\right)$ (2). Crystalline $\left({ }^{\mathrm{EMind}} \mathrm{L}\right) \mathrm{Cu}\left(\mathrm{N}_{2}\right)(\mathbf{1})^{4}(0.050 \mathrm{~g}, 0.046 \mathrm{mmol}, 1.0$ equiv.) was exported from the dinitrogen-filled drybox and exposed to air, resulting in a rapid color change in the solid state from carrot-orange to Carmine red. The solid was dissolved in hexanes and agitated for 2 minutes to ensure complete oxygenation of the solution. Subsequent removal of solvent in vacuo to afford ( $\left.{ }^{\mathrm{EMind}} \mathrm{L}\right) \mathrm{Cu}\left(\mathrm{O}_{2}\right)(2)$ as an air-stable solid in quantitative yield. Large single crystals suitable for X-ray diffraction were obtained by allowing a concentrated pentane solution of 1 to stand at $-10{ }^{\circ} \mathrm{C}$ under air overnight. Large single crystals $\left(>1 \mathrm{~cm}^{3}\right)$ could be obtained by adding drops of benzene to the crystallization conditions. ${ }^{1} \mathrm{H}$ NMR $\left(500 \mathrm{MHz}, \mathrm{C}_{6} \mathrm{D}_{6}\right): \delta 7.82(\mathrm{~s}, 1 \mathrm{H}$, fluorinated aryl $\mathrm{C}-H), 7.68(\mathrm{~s}, 2 H$, fluorinated aryl $\mathrm{C}-H), 7.12(\mathrm{~s}, 2 H$, EMind aryl $\mathrm{C}-H), 6.54(\mathrm{~d}$, $J=4.5 \mathrm{~Hz}, 2 H$, dipyrrin $\mathrm{C}-H), 6.18(\mathrm{dd}, J=4.2 \mathrm{~Hz}, 2 H$, dipyrrin $\mathrm{C}-H), 1.90(\mathrm{~d}, J=3.9 \mathrm{~Hz}, 8 H$, EMind aliphatic $\mathrm{C}-H), 1.78(\mathrm{dt}, J=9.3,6.5 \mathrm{~Hz}, 8 H$, EMind ethyl $\mathrm{C}-H), 1.61$ (apparent heptet from overlapping triplets, $J=6.9 \mathrm{~Hz}, 8 H$, EMind ethyl $\mathrm{C}-H), 1.34$ (s, $12 H$, EMind methyl $\mathrm{C}-H$ ), $1.32(\mathrm{~s}, 12 \mathrm{H}$, EMind methyl C-H), $0.97(\mathrm{t}, J=7.4 \mathrm{~Hz}, 12 H$, EMind ethyl $\mathrm{C}-H), 0.62(\mathrm{t}, J=7.3$ $\mathrm{Hz}, 12 H$, EMind ethyl $\mathrm{C}-H) .{ }^{13} \mathrm{C}\left\{{ }^{1} \mathrm{H}\right\}$ NMR $\left(125 \mathrm{MHz}, \mathrm{C}_{6} \mathrm{D}_{6}\right): \delta 162.26,148.86,148.59,141.90$, 139.34, 134.76, 131.46, 131.19, 130.93, 130.82, 130.66, 130.41, 128.71, 128.20, 126.32, 125.46, 124.15, 122.45, 121.98, 120.99, 119.81, 52.11, 48.50, 43.93, 34.26, 32.85, 32.50, 31.66, 9.29, 8.62. ${ }^{19} \mathrm{~F}$ NMR $\left(470 \mathrm{MHz}, \mathrm{C}_{6} \mathrm{D}_{6}\right): \delta-62.27$ (s, fluorinated aryl $\left.\mathrm{CF}_{3}\right)$. UV/vis $\left(\mathrm{C}_{6} \mathrm{H}_{14}\right) \lambda_{\max } / \mathrm{cm}^{-1}\left(\varepsilon / \mathrm{M}^{-1}\right.$ $\mathrm{cm}^{-1}$ ): 520 (59,000), 490 (21,700), 360 (7,000), 310 (10,000). Anal. Calc. for $\mathrm{C}_{65} \mathrm{H}_{81} \mathrm{CuF}_{6} \mathrm{~N}_{2} \mathrm{O}_{2}: \mathrm{C}$ 70.98, H 7.42, N 2.55; Found: C 70.95, H 7.11, N 2.47. HRMS (ESI ${ }^{+}$: $m / z$ Calc. 1098.5462 $\left[\mathrm{C}_{65} \mathrm{H}_{81} \mathrm{CuF}_{6} \mathrm{~N}_{2} \mathrm{O}_{2}\right]^{+}$, Found $1098.5493[\mathrm{M}]^{+}$.

Note I: Under an atmosphere of $\mathrm{N}_{2}$ in benzene, trace formation of ( $\left.{ }^{\mathrm{EMind}} \mathrm{L}\right) \mathrm{Cu}\left(\mathrm{C}_{6} \mathrm{H}_{6}\right)$ from $\left.{ }^{\text {EMind }} \mathrm{L}\right) \mathrm{Cu}\left(\mathrm{O}_{2}\right)(2)$ is observed ( $c$. $10 \%$ ), attributed to an equilibrium favoring dioxygen ligation to the copper center. Nonetheless, air exposure of this mixture affords quantitative conversion to 2.

Note II: ( $\left.{ }^{\text {EMind }} \mathrm{L}\right) \mathrm{Cu}\left({ }^{18} \mathrm{O}_{2}\right)\left(\mathbf{2 - 1 8} \mathbf{O}_{2}\right)$ was prepared by exposure of a degassed J. Young NMR tube containing in situ generated ( $\left.{ }^{\mathrm{EMind}} \mathrm{L}\right) \mathrm{Cu}\left(\mathrm{N}_{2}\right)$ in $\mathrm{C}_{6} \mathrm{D}_{12}$ to an atmosphere of ${ }^{18} \mathrm{O}_{2}$, resulting in effervescence and a color change to red. The contents of the tube were subsequently lyophilized, and Resonance Raman measurements were subsequently conducted on the solid sample without exposure to ambient air, noting facile exchange with ${ }^{16} \mathrm{O}_{2}$ from air, supported by independent Resonance Raman measurements. Due to facile ${ }^{16} \mathrm{O}_{2} /{ }^{18} \mathrm{O}_{2}$ exchange under air, HRMS on 2-18 $\mathbf{O}_{2}$ was not conducted. 


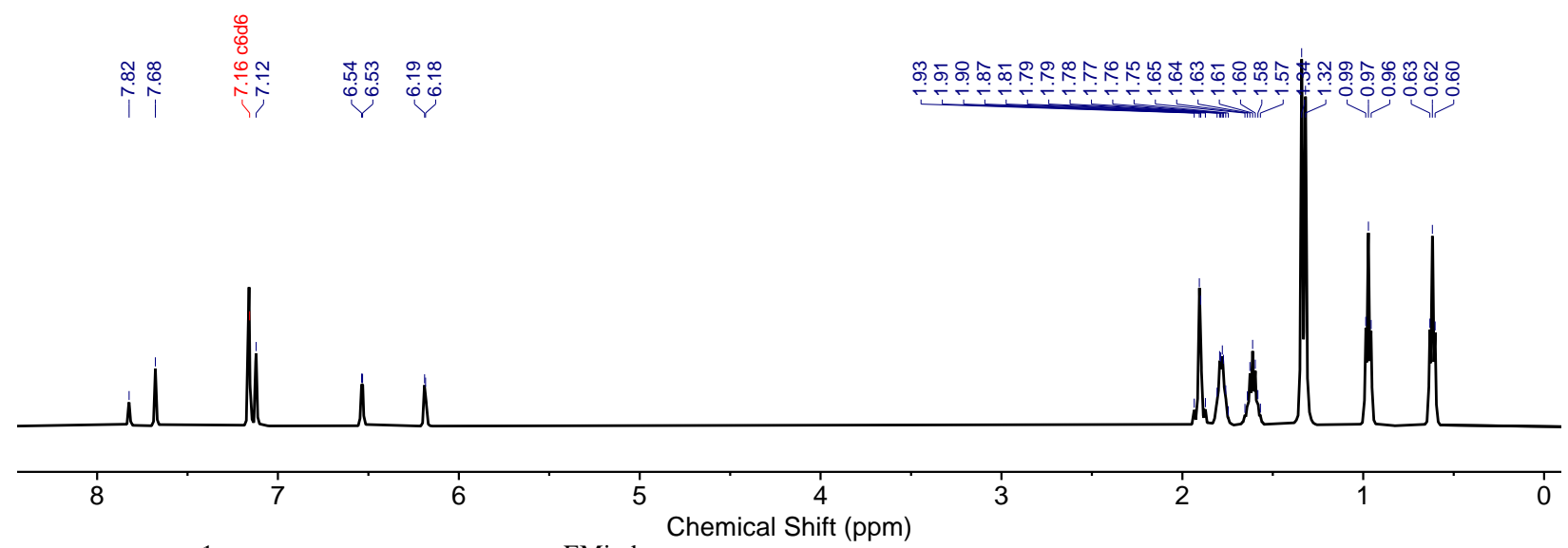

Figure S1. ${ }^{1} \mathrm{H}$ NMR spectrum of $\left({ }^{\mathrm{EMind}} \mathrm{L}\right) \mathrm{Cu}\left(\mathrm{O}_{2}\right)(\mathbf{2}),\left(500 \mathrm{MHz}, \mathrm{C}_{6} \mathrm{D}_{6}\right)$.

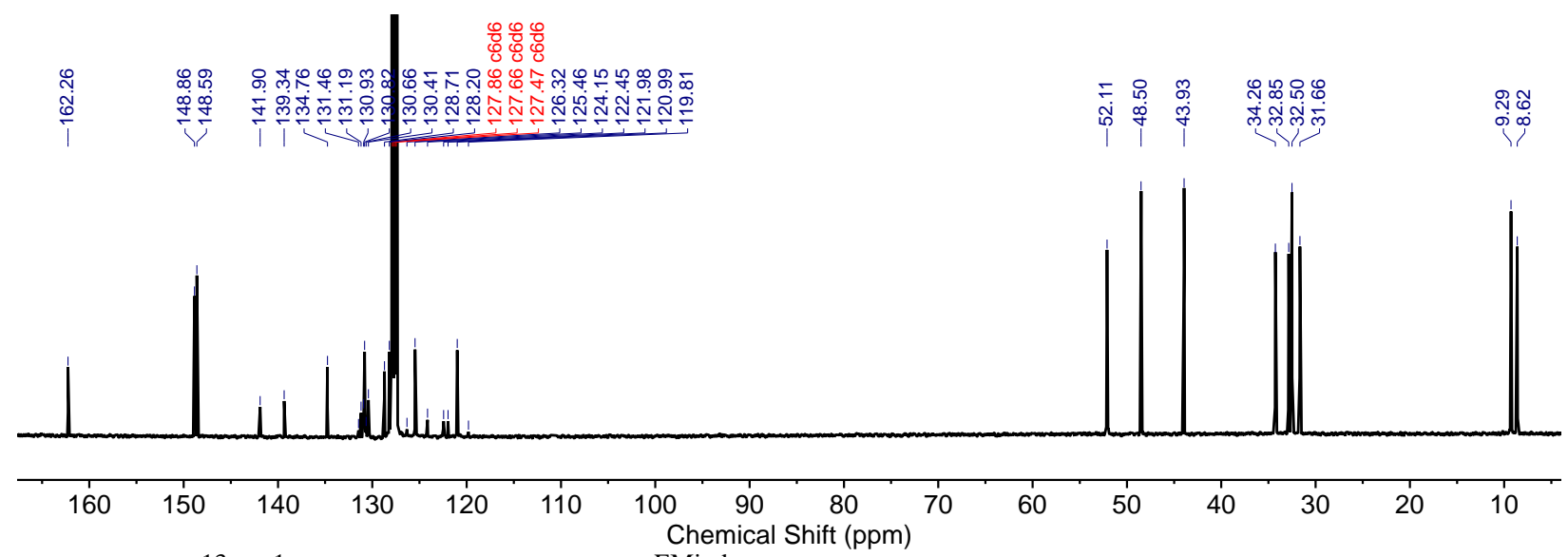

Figure S2. ${ }^{13} \mathrm{C}\left\{{ }^{1} \mathrm{H}\right\} \mathrm{NMR}$ spectrum of $\left({ }^{\mathrm{EMind}} \mathrm{L}\right) \mathrm{Cu}\left(\mathrm{O}_{2}\right)(2),\left(125 \mathrm{MHz}, \mathrm{C}_{6} \mathrm{D}_{6}\right)$.

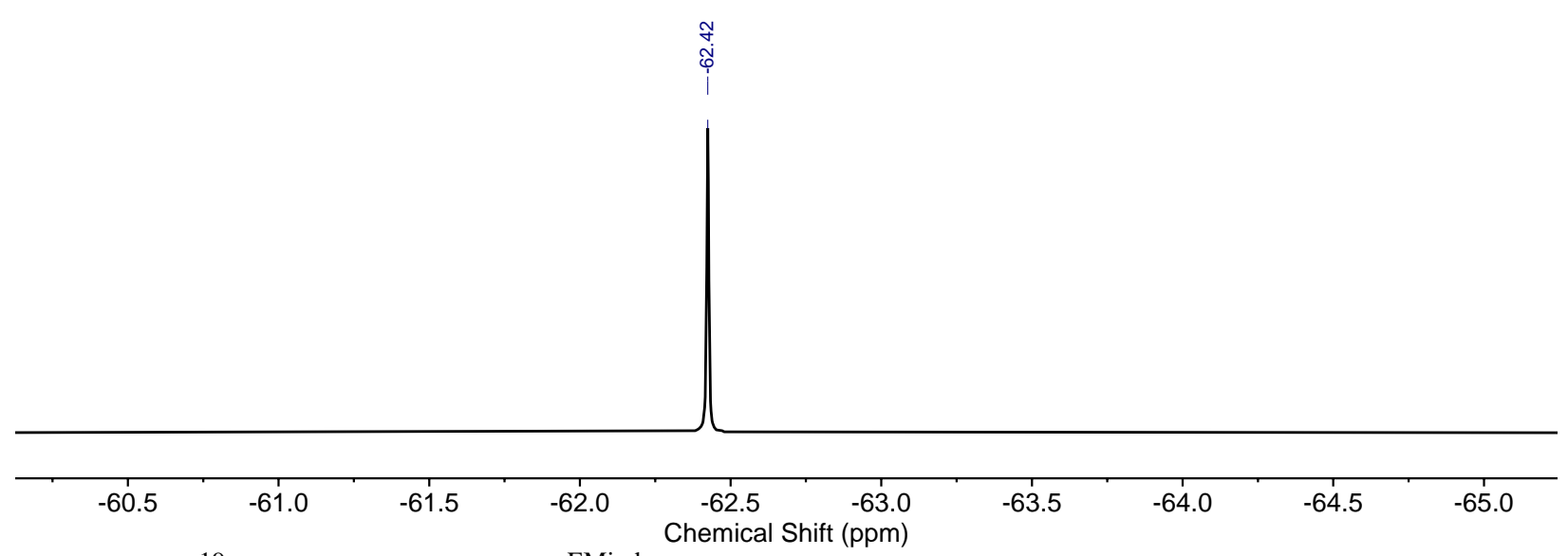

Figure S3. ${ }^{19} \mathrm{~F}$ NMR spectrum of $\left({ }^{\mathrm{EMind}} \mathrm{L}\right) \mathrm{Cu}\left(\mathrm{O}_{2}\right)(\mathbf{2}),\left(470 \mathrm{MHz}, \mathrm{C}_{6} \mathrm{D}_{6}\right)$. 


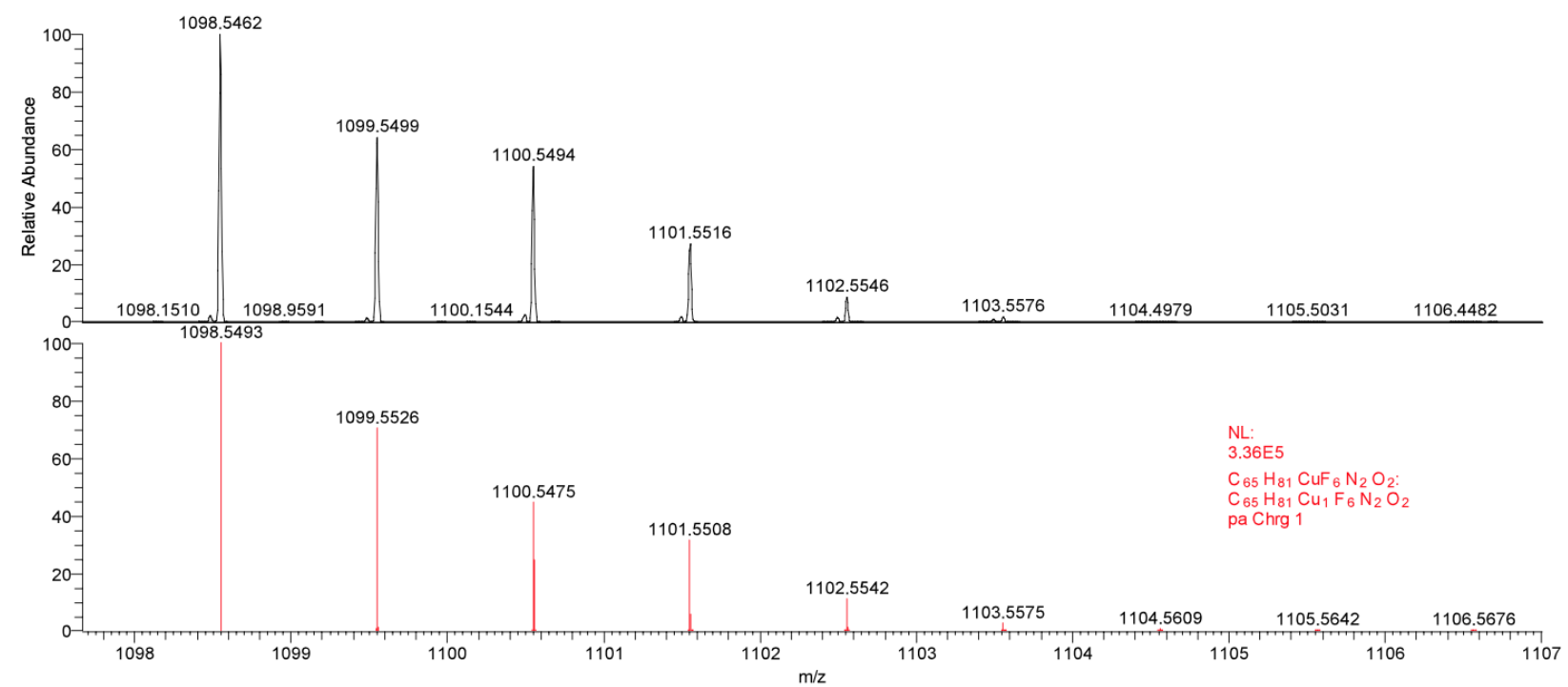

Figure S4. High-resolution mass spectrum of ( $\left.{ }^{\mathrm{EMind}} \mathrm{L}\right) \mathrm{Cu}\left(\mathrm{O}_{2}\right)$ (2) (top, black) with simulation (bottom, red), revealing the expected isotopic pattern. 


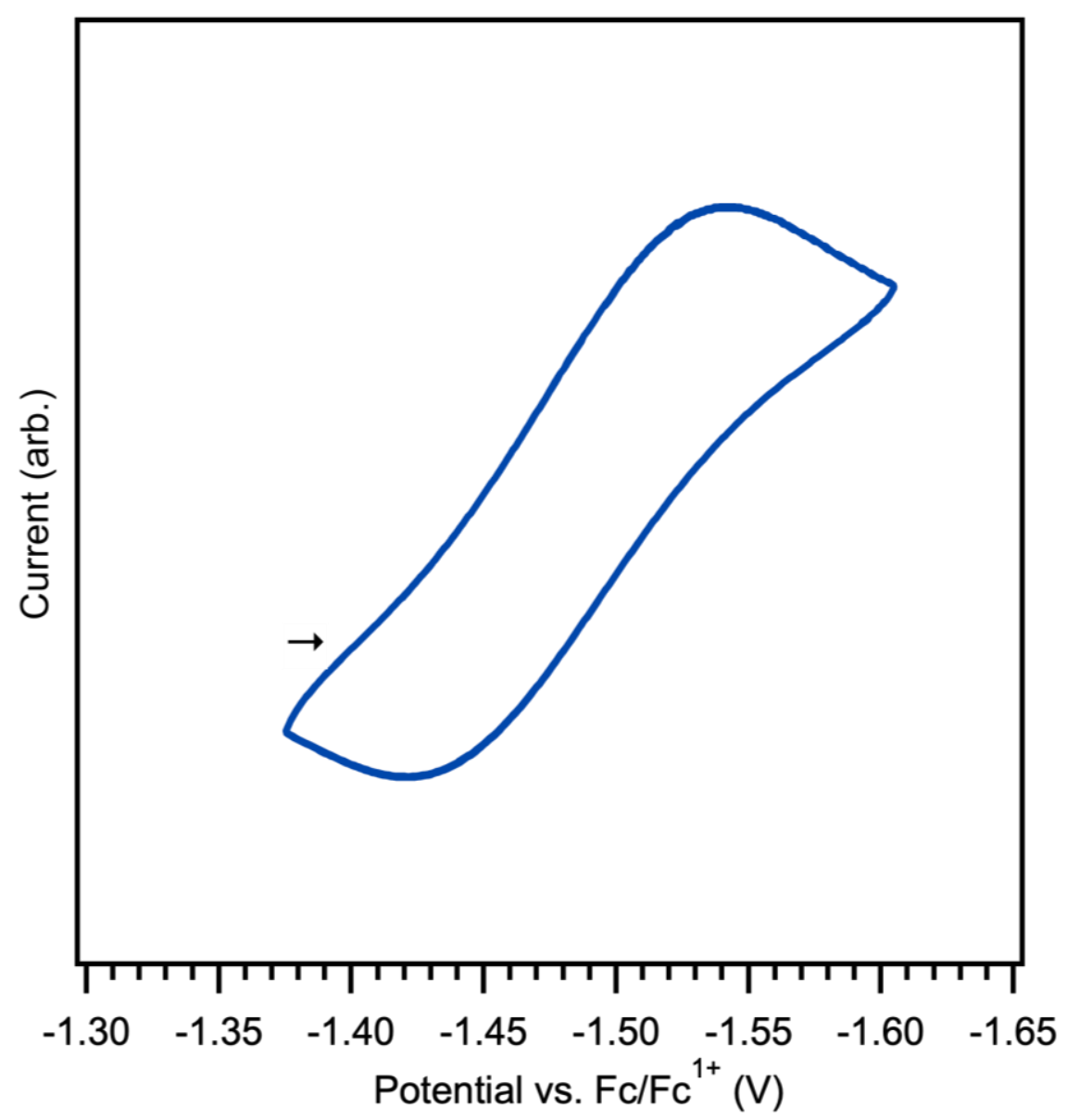

Figure S5. Quasi-reversible one-electron reduction of $\left({ }^{\mathrm{EMind}} \mathrm{L}\right) \mathrm{Cu}\left(\mathrm{O}_{2}\right)(2),\left(\mathrm{E}_{1 / 2}=-1.48 \mathrm{~V}\right.$ vs. $\mathrm{Fc} / \mathrm{Fc}^{1+}$ ) at a scan rate of $100 \mathrm{mV} \mathrm{s}^{-1}$. The data was recorded in 1,2-difluorobenzene under $\mathrm{N}_{2}$ at a concentration of ca. $0.01 \mathrm{mM}$, with glassy carbon, Pt-wire, and Ag-wire as the working, counter, and reference electrodes, respectively. Arrows denote initial scan direction. Saturated tetrabutylammonium hexafluorophosphate $\left(\left[{ }^{\mathrm{n}} \mathrm{Bu}_{4} \mathrm{~N}\right]\left[\mathrm{PF}_{6}\right]\right)$ solutions of $0.2 \mathrm{M}$ in 1,2difluorobenzene were prepared before each experiment in the presence of molecular sieves to remove trace water. No background reaction between $\mathbf{2}$ and the electrolyte was observed. 


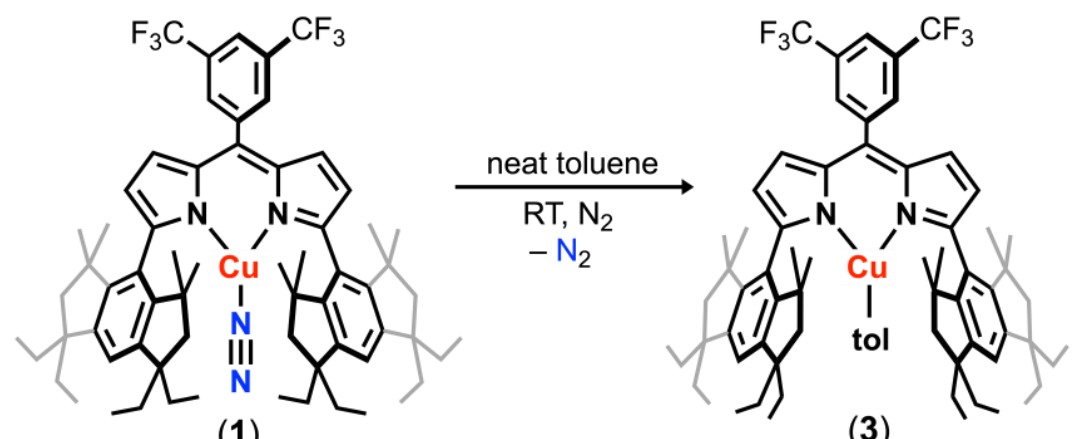

(1)

(3)

( $\left.{ }^{\text {EMind }} \mathbf{L}\right) \mathbf{C u}$ (toluene) (3). In a dinitrogen-filled drybox, dissolution of ( $\left.{ }^{\text {EMind }} \mathrm{L}\right) \mathrm{Cu}\left(\mathrm{N}_{2}\right)(\mathbf{1})^{4}(0.050$ $\mathrm{g}, 0.046 \mathrm{mmol}, 1.0$ equiv.) in toluene $(1 \mathrm{~mL})$ afforded rapid effervescence with a color change to red-orange. The mixture was agitated for 30 seconds, followed by removal of solvent in vacuo to afford $\left({ }^{\text {EMind }} \mathrm{L}\right) \mathrm{Cu}$ (toluene) (3) in quantitative yield. ${ }^{1} \mathrm{H}$ NMR $\left(500 \mathrm{MHz}, \mathrm{C}_{7} \mathrm{D}_{8}\right): \delta 7.85(\mathrm{~s}, 1 H$, fluorinated aryl $\mathrm{C}-H), 7.75(\mathrm{~s}, 1 H$, fluorinated aryl $\mathrm{C}-H), 7.05(\mathrm{~s}, 2 H$, EMind aryl $\mathrm{C}-H), 6.50(\mathrm{~d}$, $J=4.1 \mathrm{~Hz}, 2 H$, dipyrrin $\mathrm{C}-H), 6.25(\mathrm{~d}, J=4.4 \mathrm{~Hz}, 2 H$, dipyrrin $\mathrm{C}-H), 1.85(\mathrm{~s}, 8 H$, EMind aliphatic $\mathrm{C}-H$ ), 1.74 (dt, $J=9.7,6.8 \mathrm{~Hz}, 8 H$, EMind ethyl C-H), 1.57 (ddp, $J=21.2,14.3,7.2 \mathrm{~Hz}, 8 H$, EMind ethyl $\mathrm{C}-H), 1.30$ (s, 12H, EMind methyl $\mathrm{C}-H$ ), 1.24 (s, 12H, EMind methyl $\mathrm{C}-H), 0.95$ (t, $J=7.3 \mathrm{~Hz}, 9 H$, EMind ethyl C-H), $0.87(\mathrm{t}, J=7.1 \mathrm{~Hz}, 3 H$, EMind ethyl C-H), $0.56(\mathrm{t}, J=7.2 \mathrm{~Hz}$, $12 H$, EMind ethyl $\mathrm{C}-H) .{ }^{13} \mathrm{C}\left\{{ }^{1} \mathrm{H}\right\}$ NMR $\left(100 \mathrm{MHz}, \mathrm{C}_{7} \mathrm{D}_{8}\right): \delta$ 159.48, 148.52, 148.45, 143.90, 142.98 , 139.47, 137.16, 131.61, 130.98, 130.79, 130.65, 130.32, 130.04, 127.83, 127.59, 127.35, 124.97, 124.73, 124.49, 122.17, 121.27, 120.23, 119.45, 54.19, 47.77, 43.92, 34.20, 32.48, 32.26, 31.53, 22.46, 13.94, 9.06, 9.02. $\left.{ }^{19} \mathrm{~F} \mathrm{NMR} \mathrm{(470} \mathrm{MHz,} \mathrm{C}_{7} \mathrm{D}_{8}\right): \delta-62.08$ (s, fluorinated aryl $\left.\mathrm{C} F_{3}\right)$. A satisfactory elemental analysis of $\mathbf{3}$ could not be obtained due to the instability of the complex in the absence of excess toluene under an $\mathrm{N}_{2}$ atmosphere.

Note I: The synthesis of ( $\left.{ }^{\mathrm{EMind}} \mathrm{L}\right) \mathrm{Cu}$ (toluene) (3) may be performed in air by dissolution of ( $\left.{ }^{\mathrm{EMind}} \mathrm{L}\right) \mathrm{Cu}\left(\mathrm{O}_{2}\right)(2)$ in neat toluene, as confirmed by multinuclear NMR spectroscopy. Nonetheless, we note that removal of excess toluene in the presence of trace dioxygen re-affords majority formation of $\mathbf{2}$. 


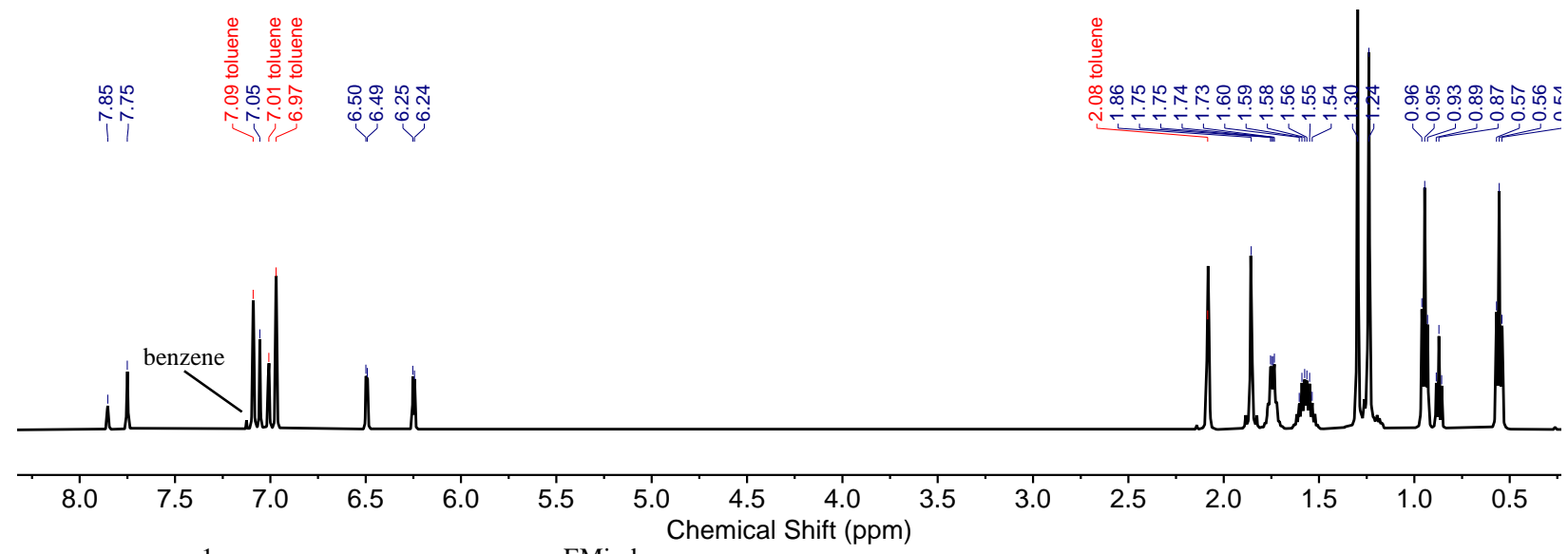

Figure S6. ${ }^{1} \mathrm{H}$ NMR spectrum of $\left({ }^{\text {EMind }} \mathrm{L}\right) \mathrm{Cu}$ (toluene) (3), (500 MHz, $\left.\mathrm{C}_{6} \mathrm{D}_{6}\right)$.

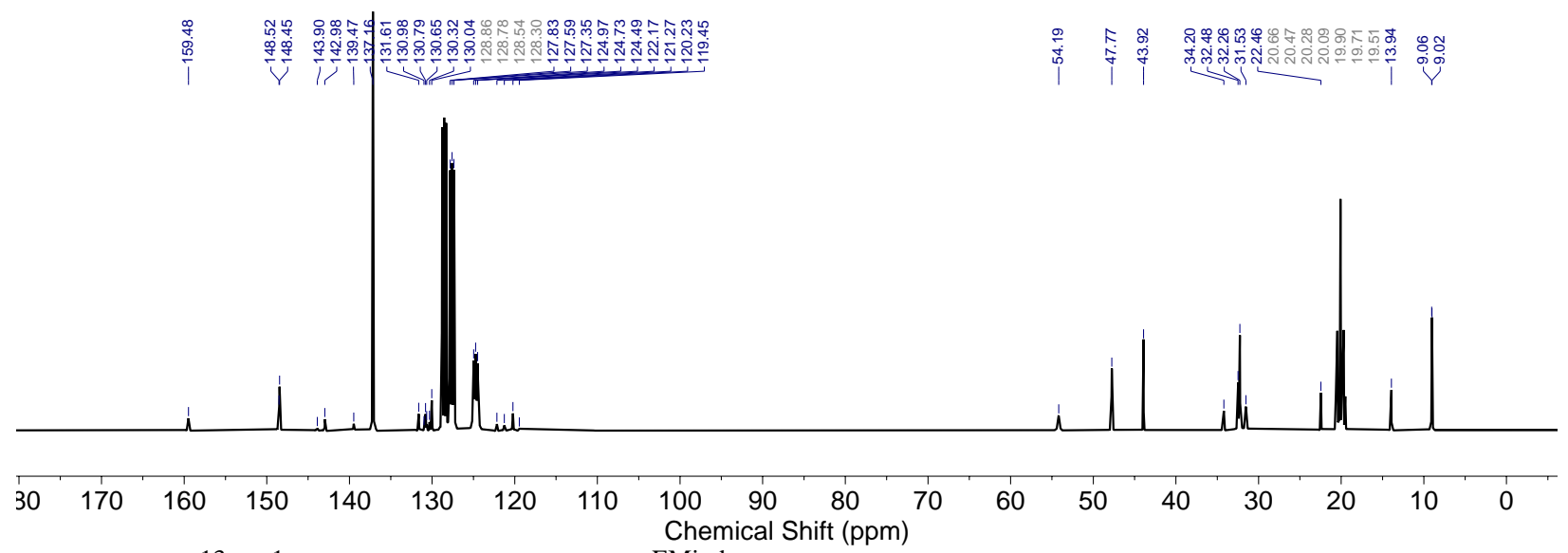

Figure S7. ${ }^{13} \mathrm{C}\left\{{ }^{1} \mathrm{H}\right\}$ NMR spectrum of ( $\left.{ }^{\text {EMind }} \mathrm{L}\right) \mathrm{Cu}$ (toluene) $(3),\left(100 \mathrm{MHz}, \mathrm{C}_{7} \mathrm{D}_{8}\right)$.

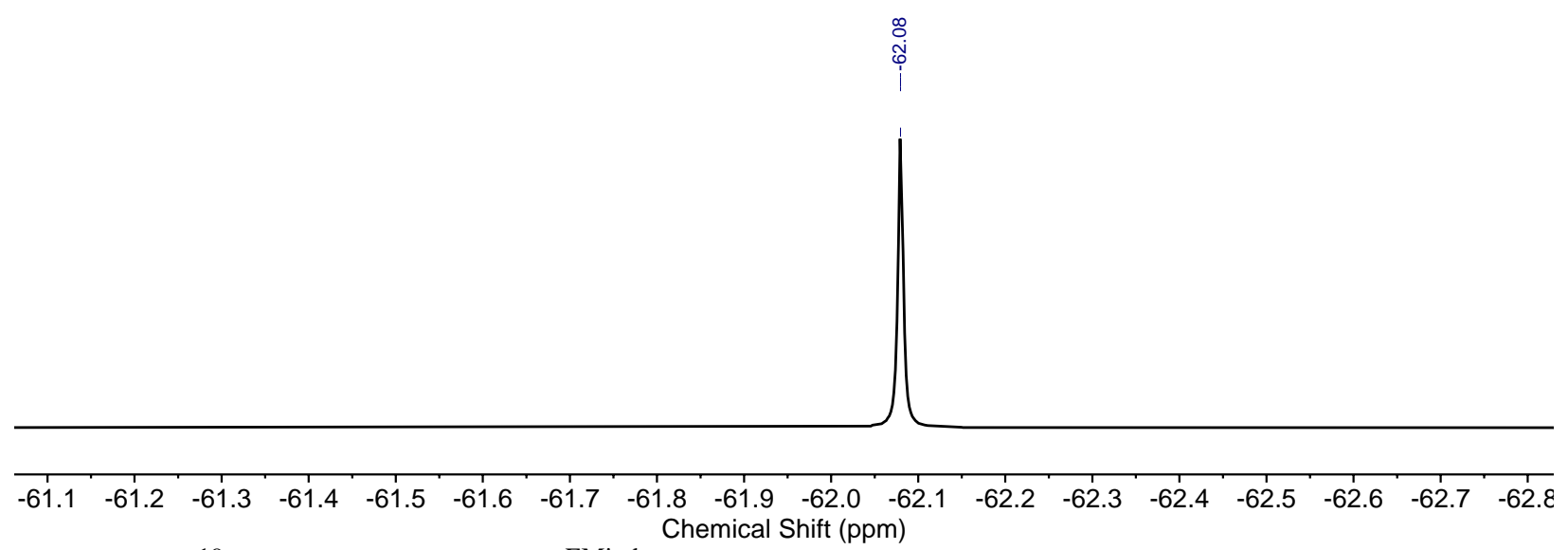

Figure S8. ${ }^{19} \mathrm{~F}$ NMR spectrum of ( $\left.{ }^{\text {EMind }} \mathrm{L}\right) \mathrm{Cu}$ (toluene) (3), (470 MHz, $\left.\mathrm{C}_{7} \mathrm{D}_{8}\right)$. 


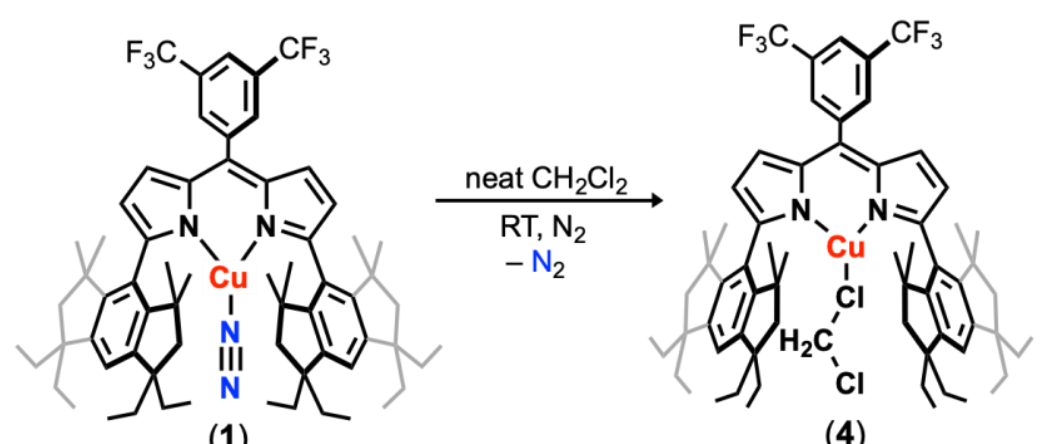

(1)

(4)

(EMind $\mathbf{L}) \mathbf{C u}\left(\mathbf{C H}_{2} \mathbf{C l}_{2}\right)$ (4). In an argon-filled drybox, dissolution of $\left({ }^{\text {EMind }} \mathrm{L}\right) \mathrm{Cu}\left(\mathrm{N}_{2}\right)(\mathbf{1})^{4}(0.050 \mathrm{~g}$, $0.046 \mathrm{mmol}, 1.0$ equiv.) into dichloromethane $(1 \mathrm{~mL})$ afforded rapid effervescence with a color change to orange-red. The mixture was physically agitated for 30 seconds, followed by removal of solvent in vacuo to afford $\left({ }^{\mathrm{EMind}} \mathrm{L}\right) \mathrm{Cu}\left(\mathrm{CH}_{2} \mathrm{Cl}_{2}\right)(\mathbf{5})$ in quantitative yield. Single crystals suitable for X-ray diffraction were obtained by reverse vapor diffusion of a dilute 100:1 pentane/dichloromethane solution of 4 into hexamethyldisiloxane at $-35^{\circ} \mathrm{C}$ under Ar over 7 days. ${ }^{1} \mathrm{H}$ NMR $\left(500 \mathrm{MHz}, \mathrm{CD}_{2} \mathrm{Cl}_{2}\right.$ ): $\delta 8.09$ (s, $2 H$, fluorinated aryl $\mathrm{C}-H$ ), 8.03 (s, $1 H$, fluorinated aryl C- $H$ ), 6.75 (br, $2 H$, EMind aryl C- $H$ ), $6.34(\mathrm{~d}, J=4.1 \mathrm{~Hz}, 2 H$, dipyrrin C- $H), 6.26(\mathrm{~d}, J=4.1 \mathrm{~Hz}$, $2 H$, dipyrrin $\mathrm{C}-H$ ), 5.32 (s, $2 H$, dichloromethane $\mathrm{C}-H$ ), 1.82 (s, $8 H$, EMind aliphatic $\mathrm{C}-H$ ), 1.52 $-1.74(\mathrm{~m}, 12 H$, EMind ethyl $\mathrm{C}-H), 1.25-1.34$ (m, 4H, EMind ethyl C- $H$ ), 1.15 (s, 12H, EMind methyl $\mathrm{C}-H), 1.10(\mathrm{~s}, 12 H$, EMind methyl $\mathrm{C}-H), 0.78-0.91$ (24H, overlapping triplet EMind ethyl $\mathrm{C}-H) .{ }^{13} \mathrm{C}\left\{{ }^{1} \mathrm{H}\right\}$ NMR $\left(100 \mathrm{MHz}, \mathrm{C}_{6} \mathrm{D}_{6}\right)$ : $\delta 158.01,148.44,148.41,142.78,142.71,138.54$, $131.74,130.98,130.73,130.40,130.07,129.74,129.16,128.33,127.71,124.99,122.90,122.28$, 121.79, 120.10, 119.57, 47.88, 43.67, 34.17, 32.82, 32.76, 32.26, 31.44, 22.38, 13.85, 9.03, 8.82. ${ }^{19} \mathrm{~F}$ NMR $\left(470 \mathrm{MHz}, \mathrm{C}_{6} \mathrm{D}_{6}\right): \delta-62.76$ (s, fluorinated aryl $\mathrm{CF}_{3}$ ). A satisfactory elemental analysis of 4 could not be obtained due to the instability of the complex in the absence of excess dichloromethane under a $\mathrm{N}_{2}$ atmosphere. 


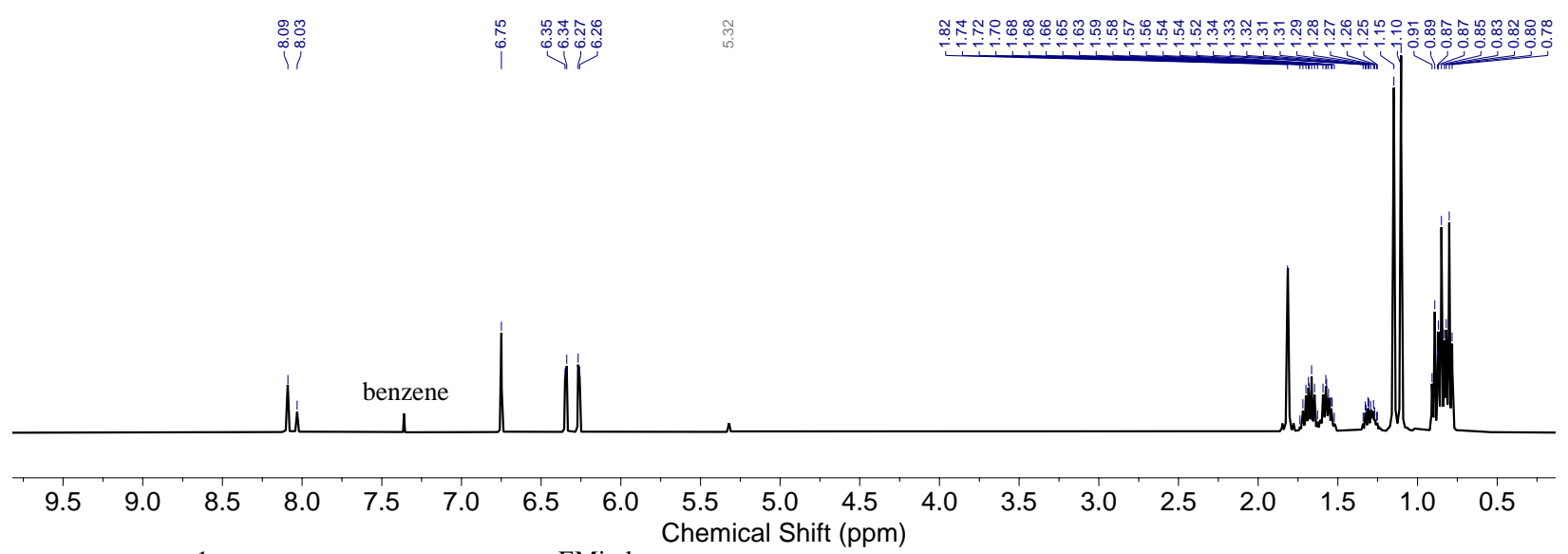

Figure S9. ${ }^{1} \mathrm{H}$ NMR spectrum of $\left({ }^{\mathrm{EMind}} \mathrm{L}\right) \mathrm{Cu}\left(\mathrm{CH}_{2} \mathrm{Cl}_{2}\right)$ (4), (500 $\left.\mathrm{MHz}, \mathrm{CD}_{2} \mathrm{Cl}_{2}\right)$.

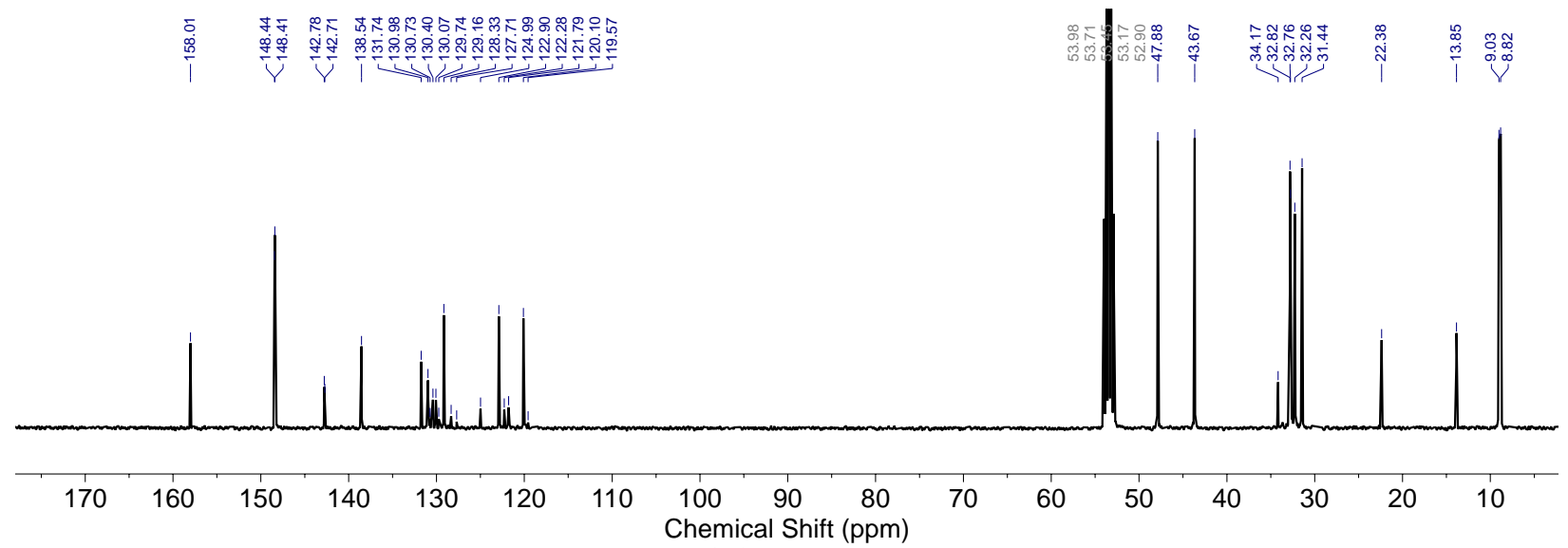

Figure S10. ${ }^{13} \mathrm{C}\left\{{ }^{1} \mathrm{H}\right\}$ NMR spectrum of $\left({ }^{\text {EMind }} \mathrm{L}\right) \mathrm{Cu}\left(\mathrm{CH}_{2} \mathrm{Cl}_{2}\right)(4),\left(125 \mathrm{MHz}, \mathrm{CD}_{2} \mathrm{Cl}_{2}\right)$.

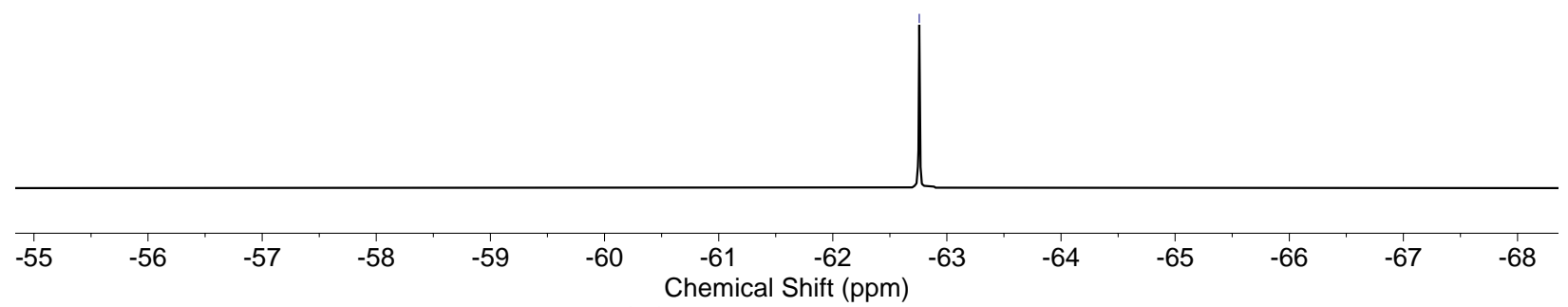

Figure S11. ${ }^{19} \mathrm{~F}$ NMR spectrum of $\left({ }^{\mathrm{EMind}} \mathrm{L}\right) \mathrm{Cu}\left(\mathrm{CH}_{2} \mathrm{Cl}_{2}\right)(4),\left(470 \mathrm{MHz}, \mathrm{C}_{6} \mathrm{D}_{6}\right)$. 


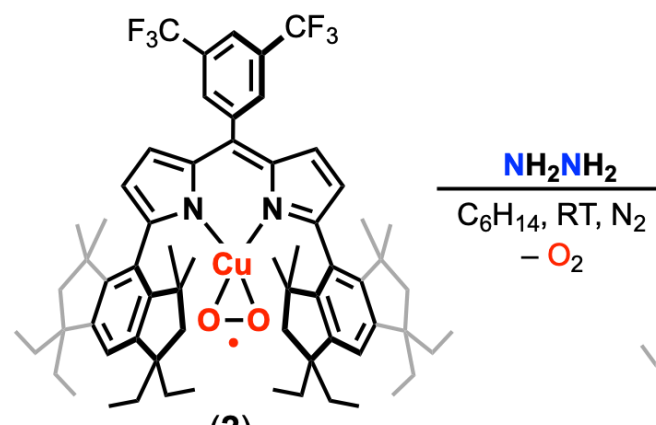

(2)

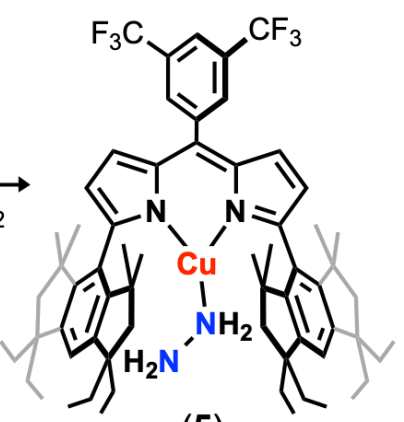

(5)

(EMind $\mathbf{L}) \mathbf{C u}\left(\mathbf{N H}_{2} \mathbf{N H}_{2}\right)$ (5). Under air, to a stirring solution of $\left({ }^{\mathrm{EMind}} \mathrm{L}\right) \mathrm{Cu}\left(\mathrm{O}_{2}\right)(\mathbf{2})(0.050 \mathrm{~g}, 0.044$ mmol, 1.0 equiv.) in hexanes ( $1 \mathrm{~mL}$ ) was added excess $\mathrm{NH}_{2} \mathrm{NH}_{2}\left(\mathrm{H}_{2} \mathrm{O}\right)$ (ca. 3.0 equiv.), resulting in rapid effervescence and a color change to bright orange. The mixture was agitated for 30 seconds, followed by removal of solvent in vacuo to afford $\left({ }^{\mathrm{EMind}} \mathrm{L}\right) \mathrm{Cu}\left(\mathrm{NH}_{2} \mathrm{NH}_{2}\right)(5)$ in quantitative yield. Single crystals suitable for X-ray diffraction were obtained by allowing a concentrated pentane solution of 5 to stand at $-35^{\circ} \mathrm{C}$ under $\mathrm{N}_{2}$ for $24 \mathrm{~h}$ or at $-10{ }^{\circ} \mathrm{C}$ under air for $72 \mathrm{~h} .{ }^{1} \mathrm{H} \mathrm{NMR}$ $\left(500 \mathrm{MHz}, \mathrm{C}_{6} \mathrm{D}_{6}\right): \delta 7.98(\mathrm{~s}, 2 H$, fluorinated aryl $\mathrm{C}-H), 7.83(\mathrm{~s}, 1 H$, fluorinated aryl $\mathrm{C}-H), 6.73$ (s, $2 H$, EMind aryl $\mathrm{C}-H), 6.58(\mathrm{~d}, J=4.3 \mathrm{~Hz}, 2 H$, dipyrrin $\mathrm{C}-H), 6.42(\mathrm{~d}, J=4.4 \mathrm{~Hz}, 2 H$, dipyrrin $\mathrm{C}-H), 2.45$ (s, $4 H$, hydrazine $\mathrm{N}-H), 1.71-1.79$ (8H, overlapping singlet EMind aliphatic $\mathrm{C}-H)$, $1.50-1.68(16 H$, overlapping multiplet from EMind ethyl $\mathrm{C}-H), 1.43$ (s, 12H, EMind methyl C$H$ ), 1.35 (s, 12H, EMind methyl C-H), $0.82(\mathrm{t}, J=7.3 \mathrm{~Hz}, 12 H$, EMind ethyl $\mathrm{C}-H), 0.76(\mathrm{t}, J=$ $7.3 \mathrm{~Hz}, 12 H$, EMind ethyl $\mathrm{C}-H) .{ }^{13} \mathrm{C}\left\{{ }^{1} \mathrm{H}\right\} \mathrm{NMR}\left(100 \mathrm{MHz}, \mathrm{C}_{6} \mathrm{D}_{6}\right): \delta 157.19,149.06,148.06$, $143.26,142.88,138.66,133.71,131.01,130.57,130.24,129.02,124.91,122.27,122.20,121.45$, $119.17,52.52,48.06,43.88,34.12,32.95,32.80,31.89,22.40,13.95,9.03 .{ }^{19} \mathrm{~F}$ NMR $(470 \mathrm{MHz}$, $\mathrm{C}_{6} \mathrm{D}_{6}$ ): -62.91 (s, fluorinated aryl $\mathrm{CF}_{3}$ ). Anal. Calc. for $\mathrm{C}_{65} \mathrm{H}_{85} \mathrm{CuF}_{6} \mathrm{~N}_{4} \bullet \mathrm{C}_{5} \mathrm{H}_{12}: \mathrm{C} 71.73, \mathrm{H} \mathrm{8.34}, \mathrm{N}$ 4.78; Found: C 72.20, H 8.28, N 5.10 (one molecule of pentane is present from bulk recrystallization and is located in the unit cell).

Note: The preparation of ( $\left.{ }^{\mathrm{EMind}} \mathrm{L}\right) \mathrm{Cu}\left(\mathrm{NH}_{2} \mathrm{NH}_{2}\right)$ (5) is similarly accomplished by treatment of ( $\left.{ }^{\mathrm{EMind}} \mathrm{L}\right) \mathrm{Cu}\left(\mathrm{N}_{2}\right)(\mathbf{1})^{4}$ with excess anhydrous hydrazine in hexanes, followed by removal of solvent to afford 6 in quantitative yield. 


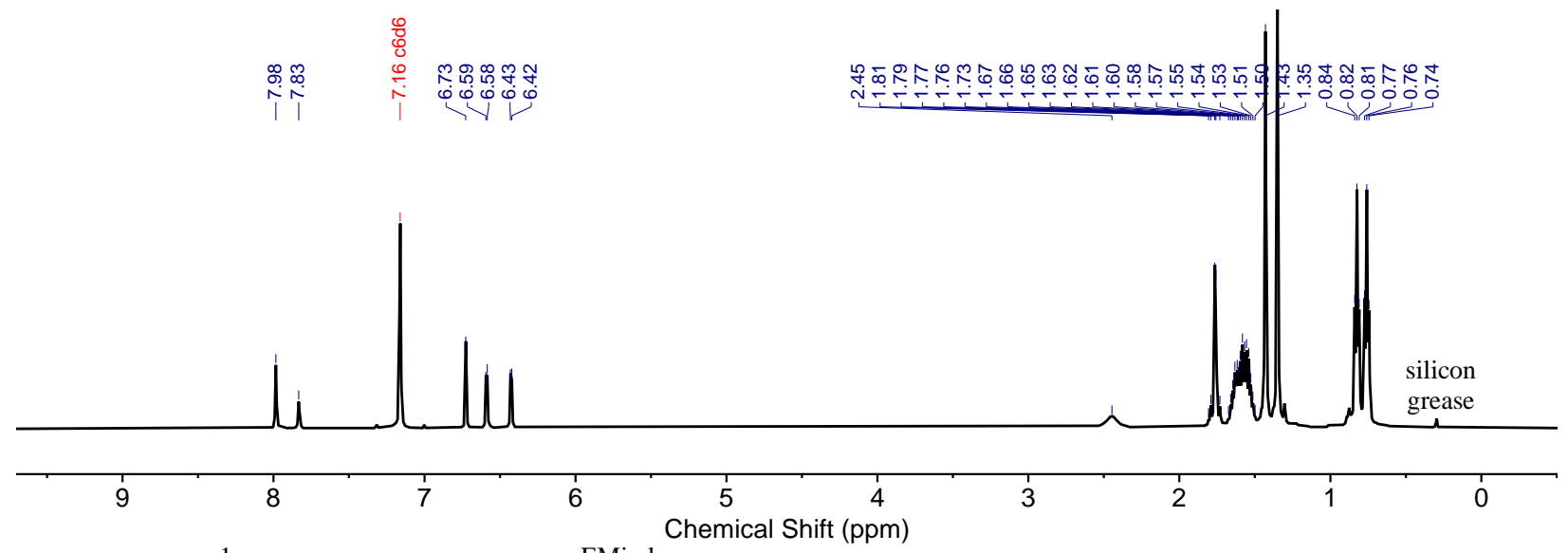

Figure S12. ${ }^{1} \mathrm{H}$ NMR spectrum of $\left({ }^{E M i n d} \mathrm{~L}\right) \mathrm{Cu}\left(\mathrm{NH}_{2} \mathrm{NH}_{2}\right)(5),\left(500 \mathrm{MHz}, \mathrm{C}_{6} \mathrm{D}_{6}\right)$.

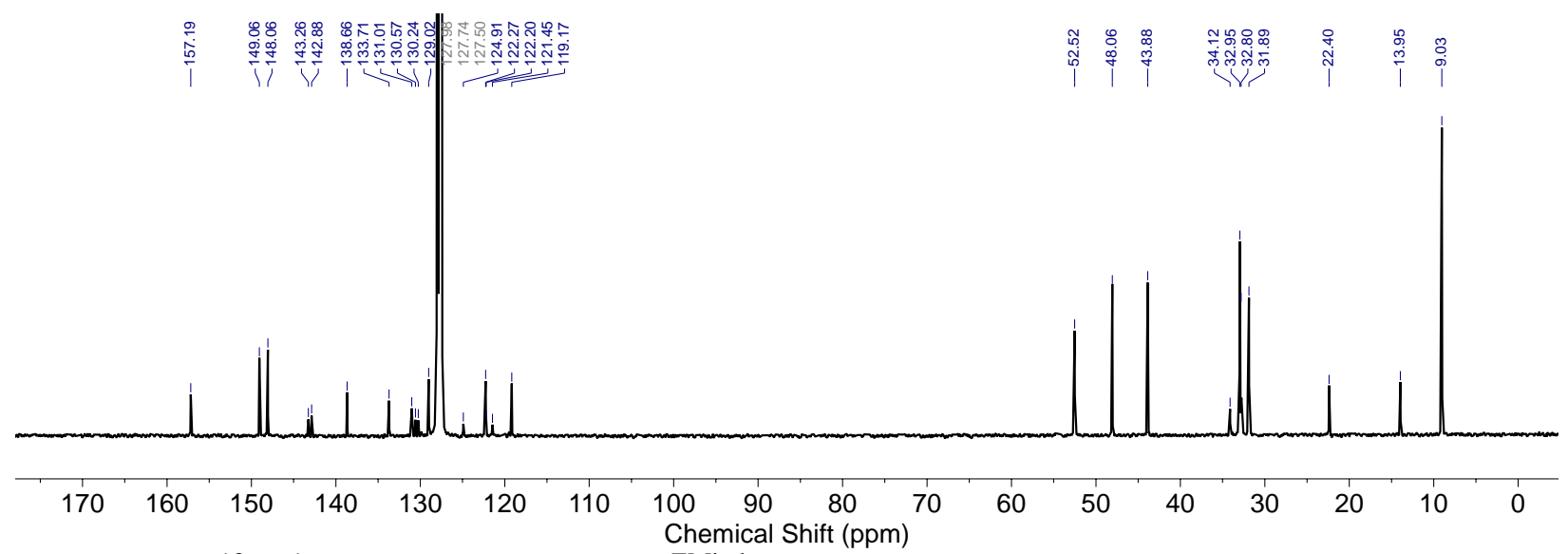

Figure S13. ${ }^{13} \mathrm{C}\left\{{ }^{1} \mathrm{H}\right\}$ NMR spectrum of $\left({ }^{\text {EMind }} \mathrm{L}\right) \mathrm{Cu}\left(\mathrm{NH}_{2} \mathrm{NH}_{2}\right)(\mathbf{5}),\left(100 \mathrm{MHz}, \mathrm{C}_{6} \mathrm{D}_{6}\right)$.

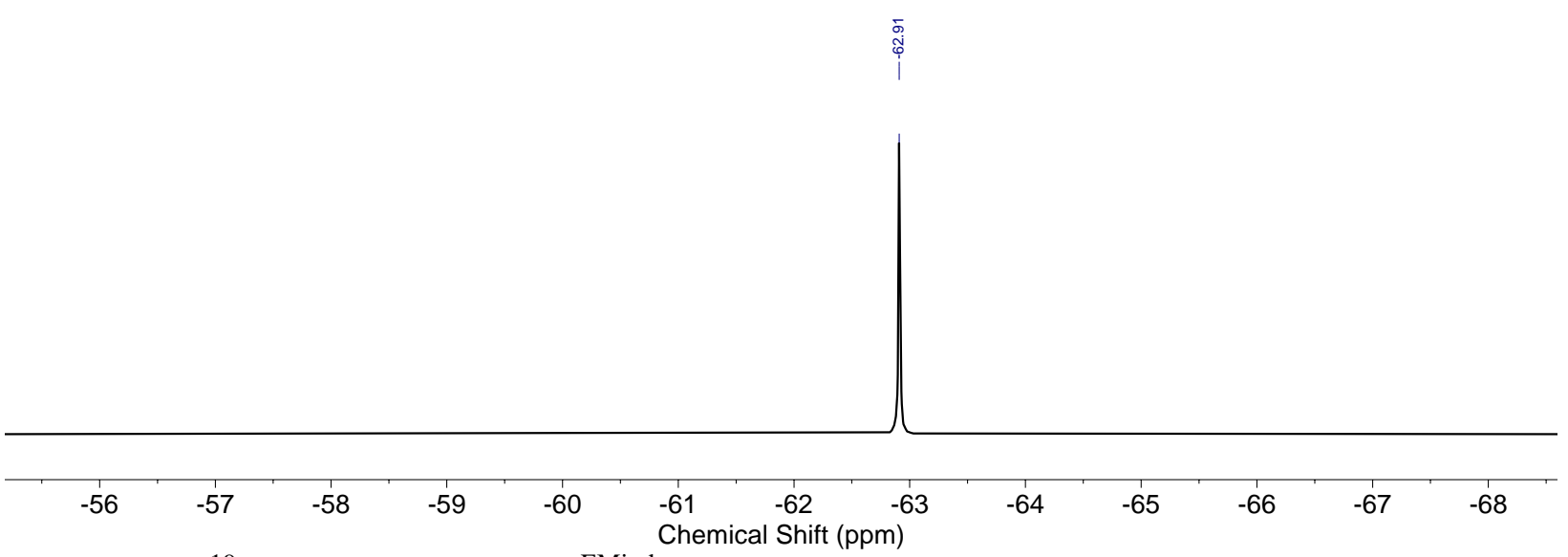

Figure S14. ${ }^{19} \mathrm{~F}$ NMR spectrum of $\left({ }^{\text {EMind }} \mathrm{L}\right) \mathrm{Cu}\left(\mathrm{NH}_{2} \mathrm{NH}_{2}\right)(\mathbf{5}),\left(470 \mathrm{MHz}, \mathrm{C}_{6} \mathrm{D}_{6}\right)$. 


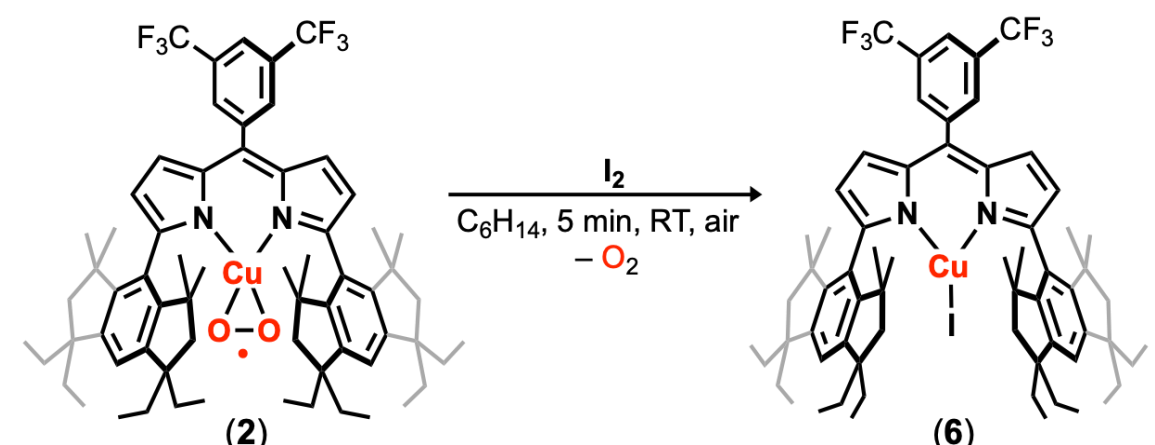

(2)

(6)

(EMind L)CuI (6). Under ambient air, elemental iodine ( $0.028 \mathrm{~g}, 0.110 \mathrm{mmol}, 1.0$ equiv.) was added as a solid to a solution of $2(0.120 \mathrm{~g}, 0.110 \mathrm{mmol}, 1.0$ equiv.) in hexanes (2 $\mathrm{mL})$, resulting in immediate effervescence and a darkening of the solution to dark pink. The solution was stirred for five minutes, followed by removal of solvent in vacuo. Removal of excess elemental iodine could be conducted either through (i) prolonged heating $\left(60^{\circ} \mathrm{C}, 24 \mathrm{~h}\right)$ under dynamic vacuum or through (ii) lyophilization, followed by suspending the residual solid in cold acetonitrile ( $\mathrm{ca} .1 \mathrm{~mL})$, rinsing over Celite, and eluting with warm benzene to afford $\left({ }^{\text {EMind }} \mathrm{L}\right) \mathrm{CuI}(\mathbf{6})$ as a brown-pink solid in essentially quantitively yield. Single crystals suitable for X-ray diffraction were obtained by allowing layering a concentrated pentane solution of 6 to stand at $-35^{\circ} \mathrm{C}$ for $72 \mathrm{~h} .{ }^{1} \mathrm{H}$ NMR $(500$ $\mathrm{MHz}, \mathrm{C}_{6} \mathrm{D}_{6}$ ): $\delta 8.41$ (v. br), 8.16 (br), 3.25 (v. br), 2.63 (br), 1.79 (v. br), 1.05 (v. br), 0.69 (v. br), 0.43 (br). ${ }^{19} \mathrm{~F}$ NMR (470 MHz, $\left.\mathrm{C}_{6} \mathrm{D}_{6}\right)$ : -62.11 . UV/vis $\left(\mathrm{C}_{6} \mathrm{H}_{14}\right) \lambda_{\max } / \mathrm{cm}^{-1}\left(\varepsilon / \mathrm{M}^{-1} \mathrm{~cm}^{-1}\right): 700(2,200)$ 525 (62,000), 500 (20,200), 380 (3,900), 310 (8,200). Anal. Calc. for $\mathrm{C}_{65} \mathrm{H}_{81} \mathrm{CuF}_{6} \mathrm{IN}_{2} \bullet \mathrm{CH}_{3} \mathrm{CN}$ : C 65.11, H 6.85, N 3.40; Found: C 65.43, H 6.58, N 3.71. 


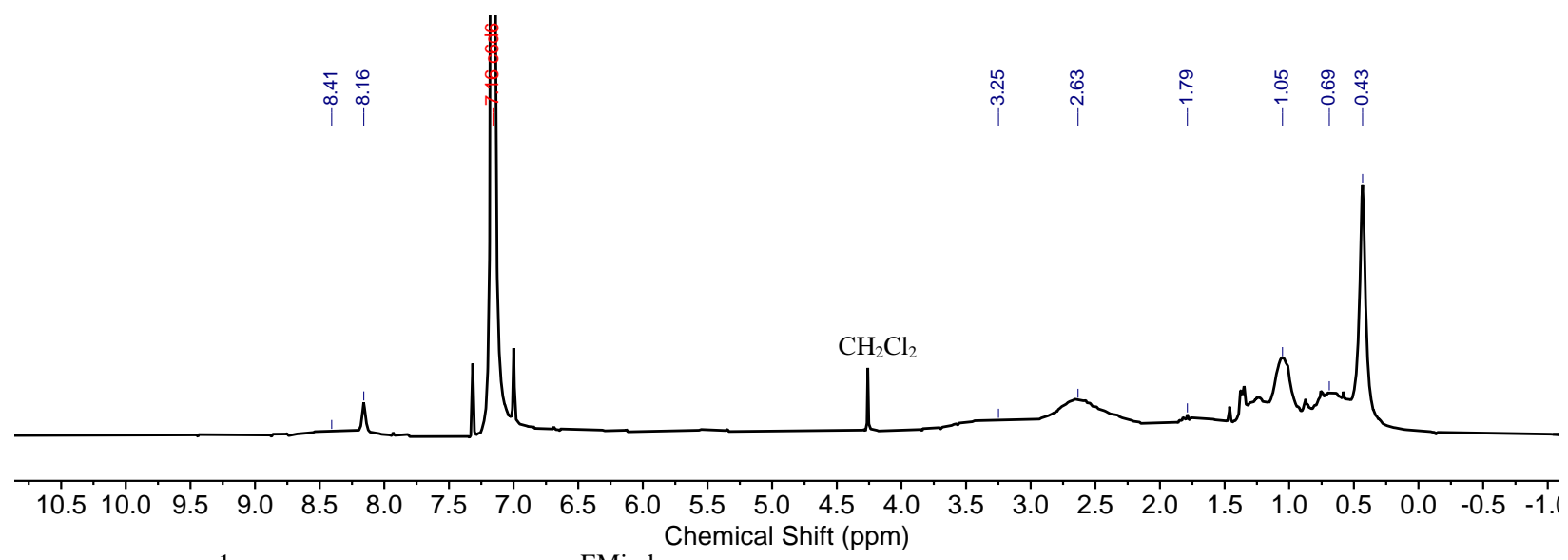

Figure S15. ${ }^{1} \mathrm{H}$ NMR spectrum of ( $\left.{ }^{\mathrm{EMind}} \mathrm{L}\right) \mathrm{CuI}(\mathbf{6}),\left(500 \mathrm{MHz}, \mathrm{C}_{6} \mathrm{D}_{6}\right)$.

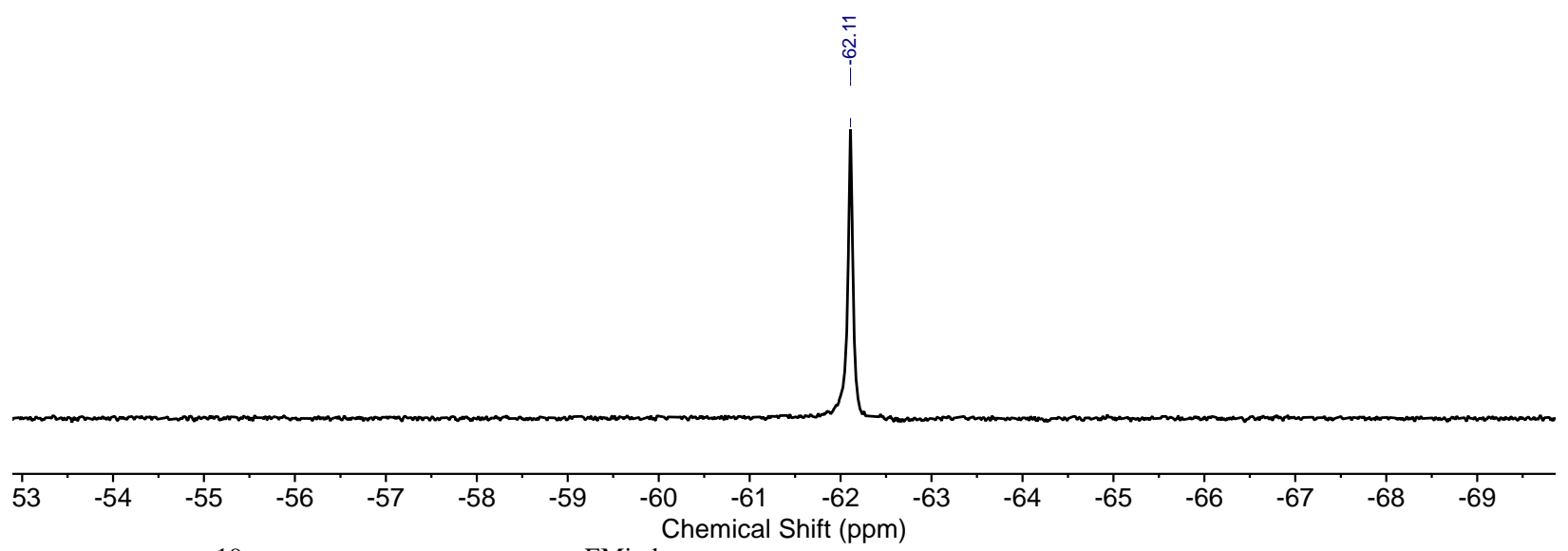

Figure S16. ${ }^{19} \mathrm{~F}$ NMR spectrum of $\left({ }^{\mathrm{EMind}} \mathrm{L}\right) \mathrm{CuI}(\mathbf{6}),\left(470 \mathrm{MHz}, \mathrm{C}_{6} \mathrm{D}_{6}\right)$. 


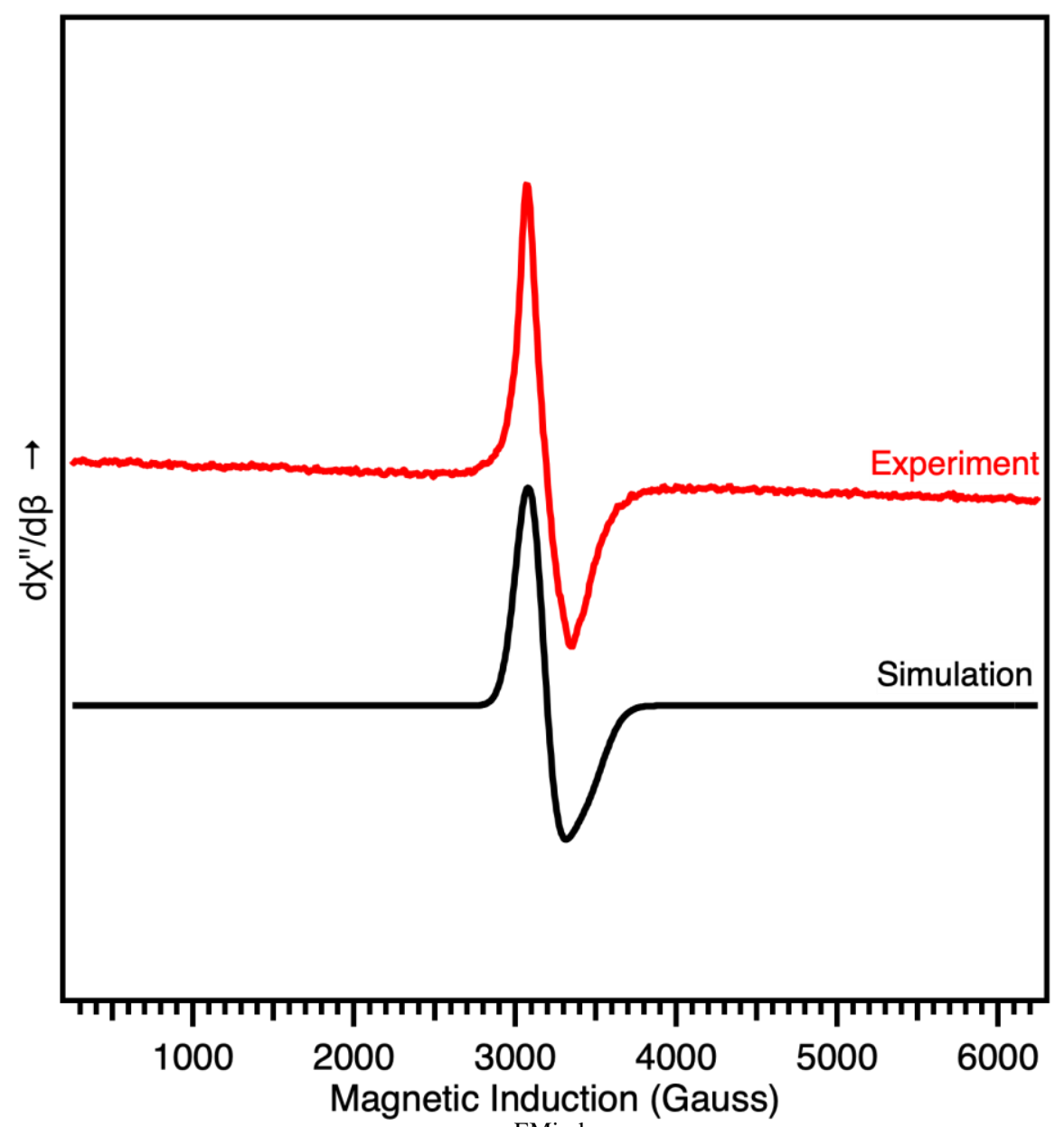

Figure S17. Solution toluene EPR spectrum of ( $\left.{ }^{\mathrm{EMind}} \mathrm{L}\right) \mathrm{CuI}(6)$ with microwave frequency of 9.857 $\mathrm{GHz}$ and $0.6325 \mathrm{~mW}$ microwave power, expanded to show an isotropic $\mathrm{S}=1 / 2$ signal (red) simulated using Easyspin (black) with the following parameters: $\mathrm{g}_{1}=2.24, \sigma \mathrm{g}_{1}=0.16 ; \mathrm{g}_{2}=2.04$, $\sigma g_{2}=0.17$. 


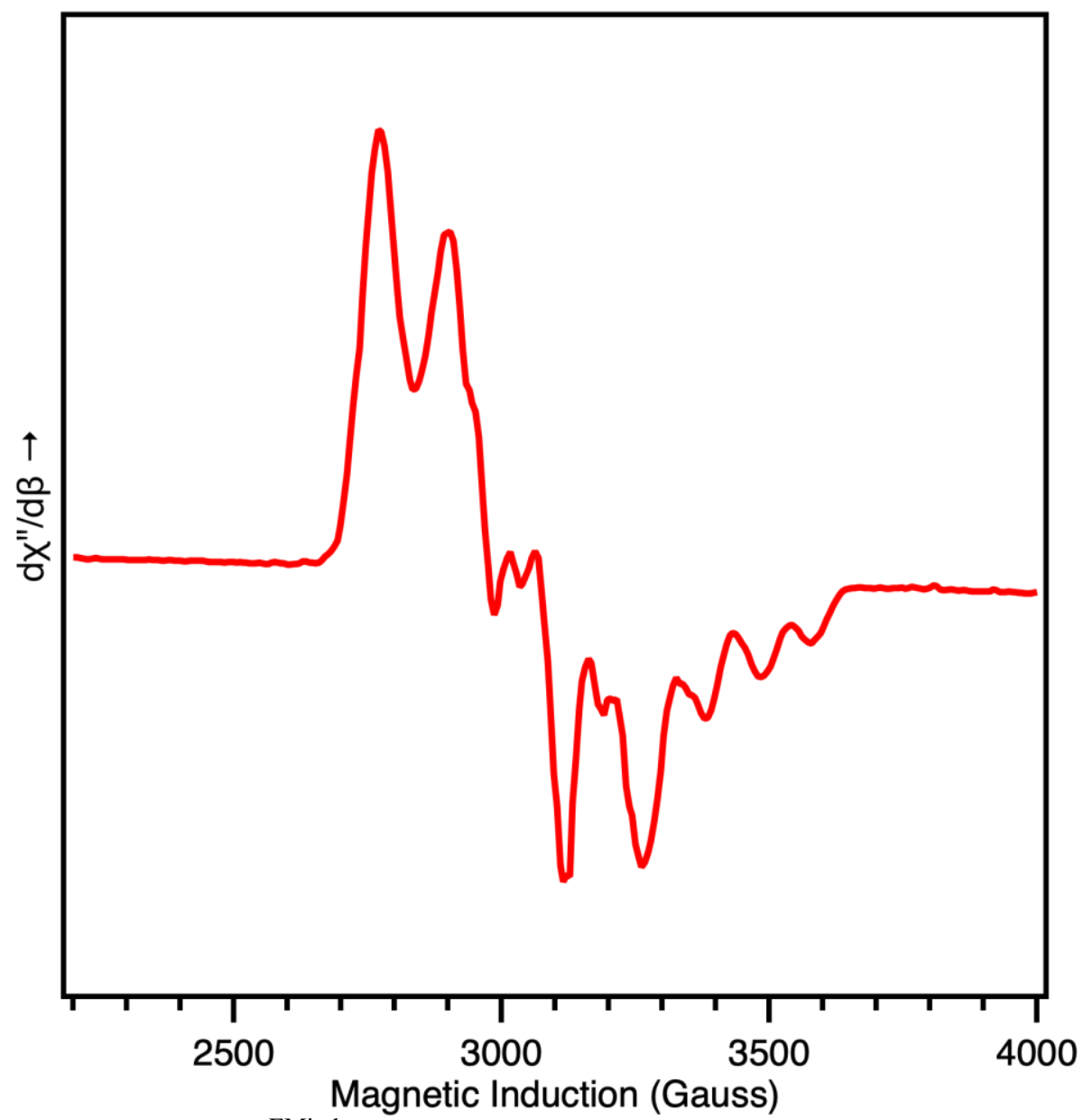

Figure S18. EPR spectrum of ( $\left.{ }^{\mathrm{EMind}} \mathrm{L}\right) \mathrm{CuI}(6)$ in frozen toluene $(77 \mathrm{~K})$ with microwave frequency of $9.332 \mathrm{GHz}$ and $0.6325 \mathrm{~mW}$ microwave power. The anisotropy is attributed to the heavy-iodine atom. 


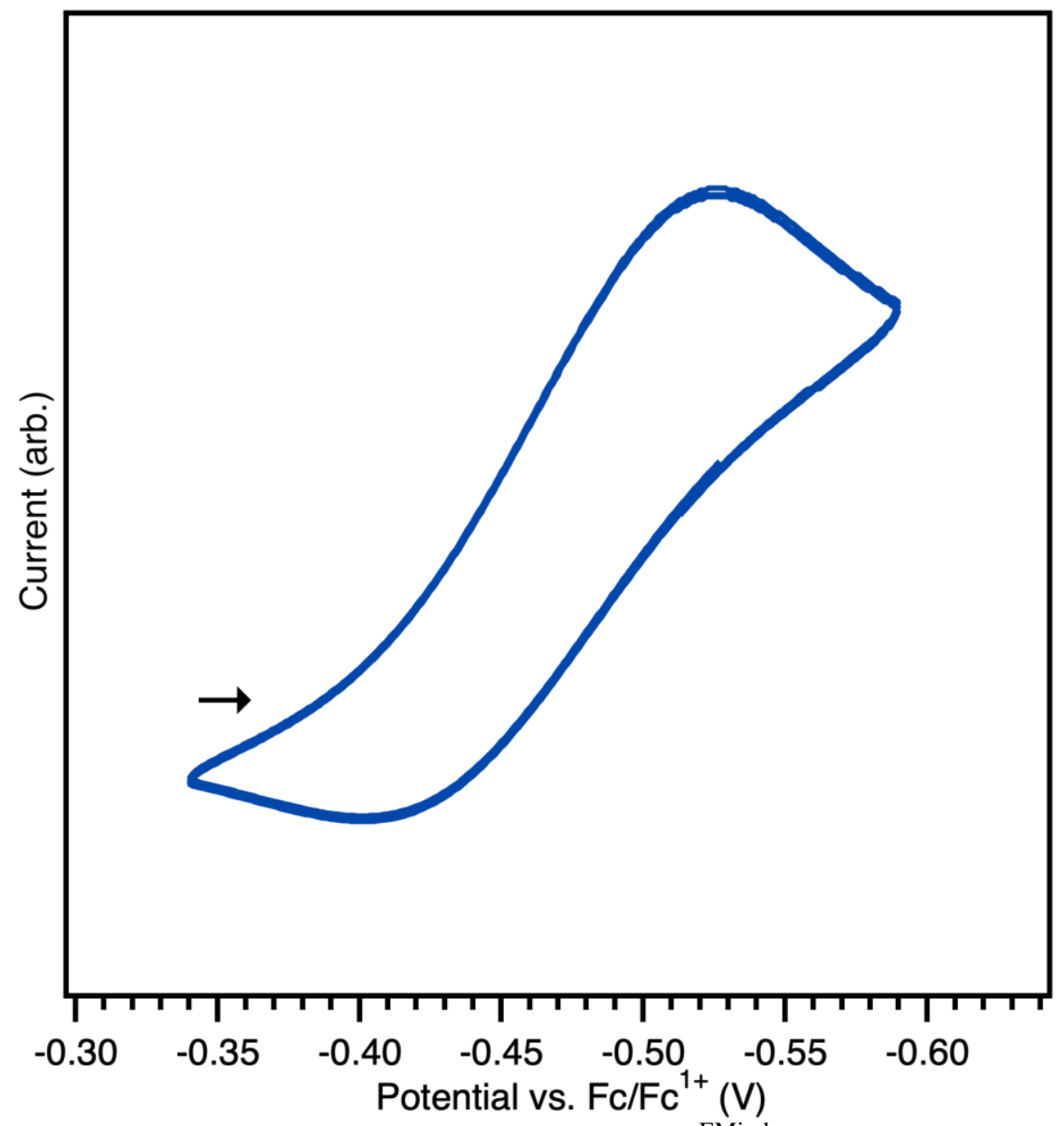

Figure S19. Quasi-reversible one-electron reduction of $\left({ }^{\mathrm{EMind}} \mathrm{L}\right) \mathrm{CuI}(\mathbf{6}),\left(\mathrm{E}_{1 / 2}=-0.46 \mathrm{~V}\right.$ vs. $\mathrm{Fc} / \mathrm{Fc}^{1+}$ ) at a scan rate of $100 \mathrm{mV} \mathrm{s}^{-1}$. The data was recorded in 1,2-difluorobenzene at a concentration of ca. $0.01 \mathrm{mM}$, with glassy carbon, Pt-wire, and Ag-wire as the working, counter, and reference electrodes, respectively. Arrows denote initial scan direction. Saturated tetrabutylammonium hexafluorophosphate $\left(\left[{ }^{n} \mathrm{Bu}_{4} \mathrm{~N}\right]\left[\mathrm{PF}_{6}\right]\right)$ solutions of $0.2 \mathrm{M}$ in 1,2difluorobenzene were prepared before each experiment in the presence of molecular sieves to remove trace water. No background reaction between $\mathbf{8}$ and the electrolyte was observed. 


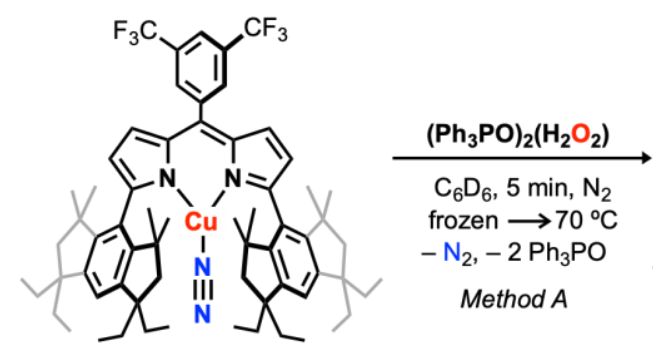

(1)

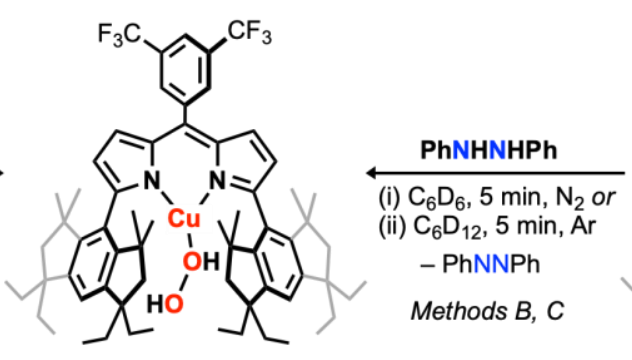

(7)

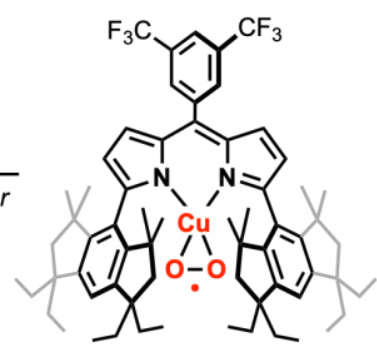

(2)

( $\left.{ }^{\mathbf{E M i n d}} \mathbf{L}\right) \mathbf{C u}\left(\mathrm{H}_{2} \mathrm{O}_{2}\right)$ (7). The in situ preparation of $\left({ }^{\mathrm{EMind}} \mathrm{L}\right) \mathrm{Cu}\left(\mathrm{H}_{2} \mathrm{O}_{2}\right)(7)$ could be achieved in three separate protocols:

Method A. In a dinitrogen-filled drybox, crystalline $\left({ }^{\text {EMind }} \mathrm{L}\right) \mathrm{Cu}\left(\mathrm{N}_{2}\right)(\mathbf{1})^{4}(0.008 \mathrm{~g}, 0.007 \mathrm{mmol}, 1.0$ equiv.) was dissolved in $\mathrm{C}_{6} \mathrm{D}_{6}(c a .0 .4 \mathrm{~mL})$, resulting in effervescence and a subtle color change to red-orange, indicative of $\left({ }^{\mathrm{EMind}} \mathrm{L}\right) \mathrm{Cu}\left(\mathrm{C}_{6} \mathrm{D}_{6}\right)$. The mixture was transferred to an NMR tube and frozen in a liquid-nitrogen chilled cold well. Upon thawing, $\left(\mathrm{Ph}_{3} \mathrm{PO}\right)_{2}\left(\mathrm{H}_{2} \mathrm{O}_{2}\right)^{2}(0.005 \mathrm{~g}, 0.008$ mmol, 1.1 equiv.) was added in $\mathrm{C}_{6} \mathrm{D}_{6}(c a .0 .3 \mathrm{~mL})$, resulting in a slight darkening of the solution. Analysis by multinuclear NMR spectroscopy revealed full consumption of ( $\left.{ }^{\text {EMind }} \mathrm{L}\right) \mathrm{Cu}\left(\mathrm{C}_{6} \mathrm{D}_{6}\right)$, accompanied by formation of triphenylphosphine oxide and formation of a new $\mathrm{Cu}$-containing product, assigned as ( $\left.{ }^{\mathrm{EMind}} \mathrm{L}\right) \mathrm{Cu}\left(\mathrm{H}_{2} \mathrm{O}_{2}\right)(7) .{ }^{1} \mathrm{H} \mathrm{NMR}\left(500 \mathrm{MHz}, \mathrm{C}_{6} \mathrm{D}_{6}\right): \delta 7.91(\mathrm{~s}, 1 \mathrm{H}$, fluorinated aryl $\mathrm{C}-H), 7.85$ (s, $1 H$, fluorinated aryl $\mathrm{C}-H), 7.04$ (broad s, $2 H$, EMind aryl $\mathrm{C}-H), 6.48(\mathrm{~d}, J=$ $4.2 \mathrm{~Hz}, 2 H$, dipyrrin $\mathrm{C}-H), 6.25(\mathrm{~d}, J=4.2 \mathrm{~Hz}, 1 H$, dipyrrin $\mathrm{C}-H), 1.56-1.91(24 H$, overlapping singlet $8 H$ from EMind aliphatic $\mathrm{C}-H$ and multiplet $16 H$ from EMind ethyl $\mathrm{C}-H$ ), 1.30 (s, 24H, EMind methyl $\mathrm{C}-H$ ), 0.89 (apparent quintet from overlapping triplets, $J=7.4 \mathrm{~Hz}, 24 \mathrm{H}$, EMind ethyl $\mathrm{C}-H$ ). ${ }^{19} \mathrm{~F}$ NMR $\left(470 \mathrm{MHz}, \mathrm{C}_{6} \mathrm{D}_{6}\right): \delta-62.36$ (s, fluorinated aryl $\mathrm{CF}_{3}$ ). Partial removal of triphenylphosphine could be achieved by filtering a just-thawed solution of 7 in minimal pentane. Control experiments reveal no background changes by NMR spectroscopy of ( $\left.{ }^{\mathrm{EMind}} \mathrm{L}\right) \mathrm{Cu}\left(\mathrm{N}_{2}\right)$ upon addition of excess triphenylphosphine. Formation of ( $\left.{ }^{\text {EMind }} \mathrm{L}\right) \mathrm{Cu}\left(\mathrm{H}_{2} \mathrm{O}\right)$ was achieved through treatment of $\left({ }^{\text {EMind }} \mathrm{L}\right) \mathrm{Cu}\left(\mathrm{N}_{2}\right)$ with stoichiometric water, which exhibited distinct ${ }^{1} \mathrm{H} /{ }^{19} \mathrm{~F} N M R$ resonances from 7 (manuscript in preparation).

Method B. In a dinitrogen-filled drybox, to a thawing solution of $\left({ }^{\text {EMind }} \mathrm{L}\right) \mathrm{Cu}\left(\mathrm{O}_{2}\right)$ (2) in $\mathrm{C}_{6} \mathrm{D}_{6}$ (containing ca. $5 \%$ ( $\left.{ }^{\mathrm{EMind}} \mathrm{L}\right) \mathrm{Cu}\left(\mathrm{N}_{2}\right)$ due to solution equilibration) was added stoichiometric 1,2diphenylhydrazine. Analysis by multinuclear $\left({ }^{1} \mathrm{H} /{ }^{19} \mathrm{~F}\right)$ NMR spectroscopy reveals consumption of ( $\left.{ }^{\text {EMind }} \mathrm{L}\right) \mathrm{Cu}\left(\mathrm{O}_{2}\right)(2)$ and formation of both $\left({ }^{\text {EMind }} \mathrm{L}\right) \mathrm{Cu}\left(\mathrm{N}_{2}\right)(\mathbf{1})$, azoarene, and a new Cu-containing product identical to that obtained from Method A. Control experiments reveal no background reaction of ( $\left.{ }^{\text {EMind }} \mathrm{L}\right) \mathrm{Cu}\left(\mathrm{O}_{2}\right)$ with either 1,2-diphenylhydrazine or the corresponding azoarene.

Method $C$. In an argon-filled drybox, to a solution of $\left({ }^{\text {EMind }} \mathrm{L}\right) \mathrm{Cu}\left(\mathrm{O}_{2}\right)(2)$ in $\mathrm{C}_{6} \mathrm{D}_{12}$ was added stoichiometric 1,2-diphenylhydrazine. Analysis by multinuclear $\left({ }^{1} \mathrm{H} /{ }^{19} \mathrm{~F}\right) \mathrm{NMR}$ spectroscopy reveals consumption of ( $\left.{ }^{\mathrm{EMind}} \mathrm{L}\right) \mathrm{Cu}\left(\mathrm{O}_{2}\right)(2)$ and formation of a new diamagnetic species, identical to the product from Method A. Control experiments reveal no background reaction of ( $\left.{ }^{\mathrm{EMind}} \mathrm{L}\right) \mathrm{Cu}\left(\mathrm{O}_{2}\right)(2)$ with either 1,2-diphenylhydrazine or the corresponding azoarene. 
Note I - The aliphatic resonance attributed to the EMind methyl $\mathrm{C}-\mathrm{H}$ is a single resonance ( $24 \mathrm{H}$ integration) in $\left({ }^{\mathrm{EMind}} \mathrm{L}\right) \mathrm{Cu}\left(\mathrm{H}_{2} \mathrm{O}_{2}\right)(7)$, contrasting the two singlet resonances in $\left({ }^{\mathrm{EMind}} \mathrm{L}\right) \mathrm{Cu}\left(\mathrm{C}_{6} \mathrm{D}_{6}\right)$ for the same EMind methyl $\mathrm{C}-\mathrm{H}(2 \times 12 \mathrm{H}$ integration $)$. This chemical difference indicates that 7 is a distinct diamagnetic species compared to $\mathbf{1}$.

Note II - Allowing solutions of ( $\left.{ }^{\mathrm{EMind}} \mathrm{L}\right) \mathrm{Cu}\left(\mathrm{H}_{2} \mathrm{O}_{2}\right)$ (7) to stand in $\mathrm{C}_{6} \mathrm{D}_{6}$ over hours affords increasing amounts of ( $\left.{ }^{\mathrm{EMind}} \mathrm{L}\right) \mathrm{H}$, attributed to the acidicity of $\mathrm{H}_{2} \mathrm{O}_{2}$ resulting in proteodemetallation. Attempts at crystallizing $\mathbf{7}$ under $\mathrm{N}_{2}$ as concentrated solutions of neat pentane has resulted in formation of crystalline $\mathbf{1}$.

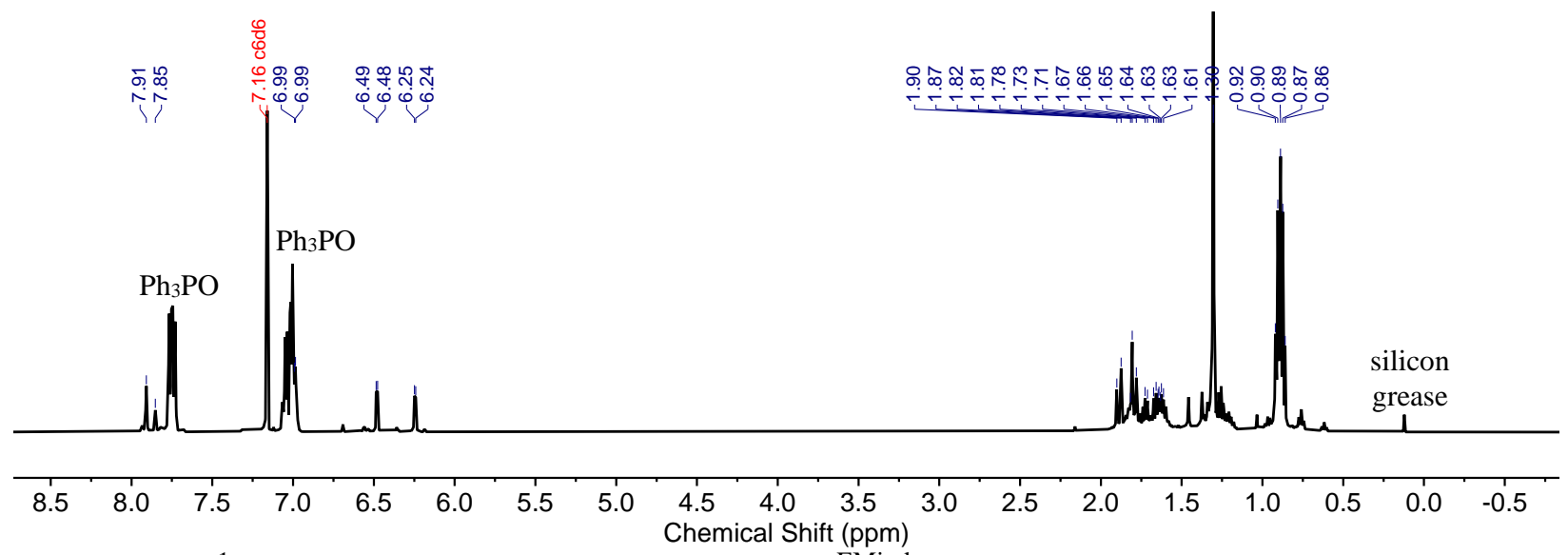

Figure S20. ${ }^{1} \mathrm{H}$ NMR spectrum of in situ prepared ( $\left.{ }^{\mathrm{EMind}} \mathrm{L}\right) \mathrm{Cu}\left(\mathrm{H}_{2} \mathrm{O}_{2}\right)(7),\left(500 \mathrm{MHz}, \mathrm{C}_{6} \mathrm{D}_{6}\right)$.

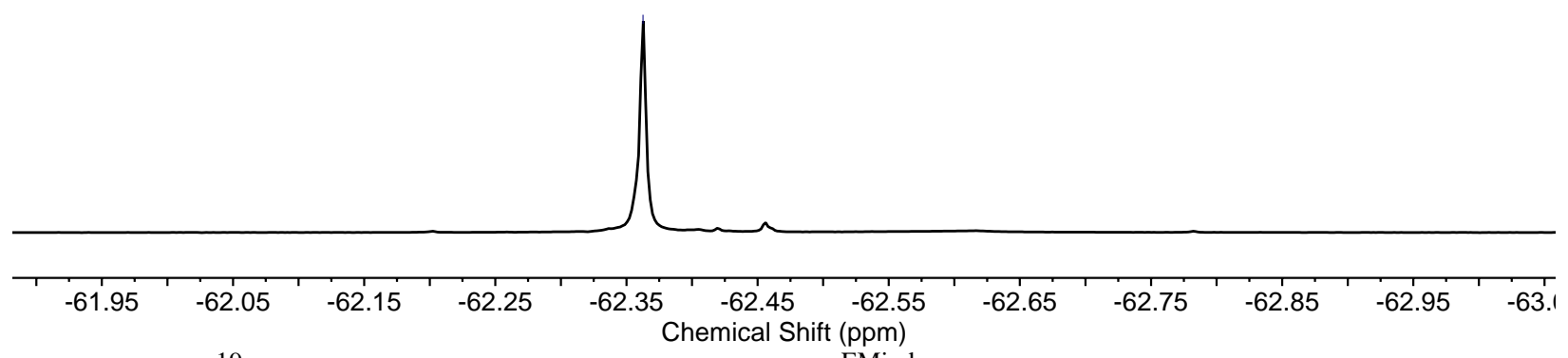

Figure S21. ${ }^{19} \mathrm{~F}$ NMR spectrum of in situ prepared ( $\left.{ }^{\mathrm{EMind}} \mathrm{L}\right) \mathrm{Cu}\left(\mathrm{H}_{2} \mathrm{O}_{2}\right)(7),\left(470 \mathrm{MHz}, \mathrm{C}_{6} \mathrm{D}_{6}\right)$. 


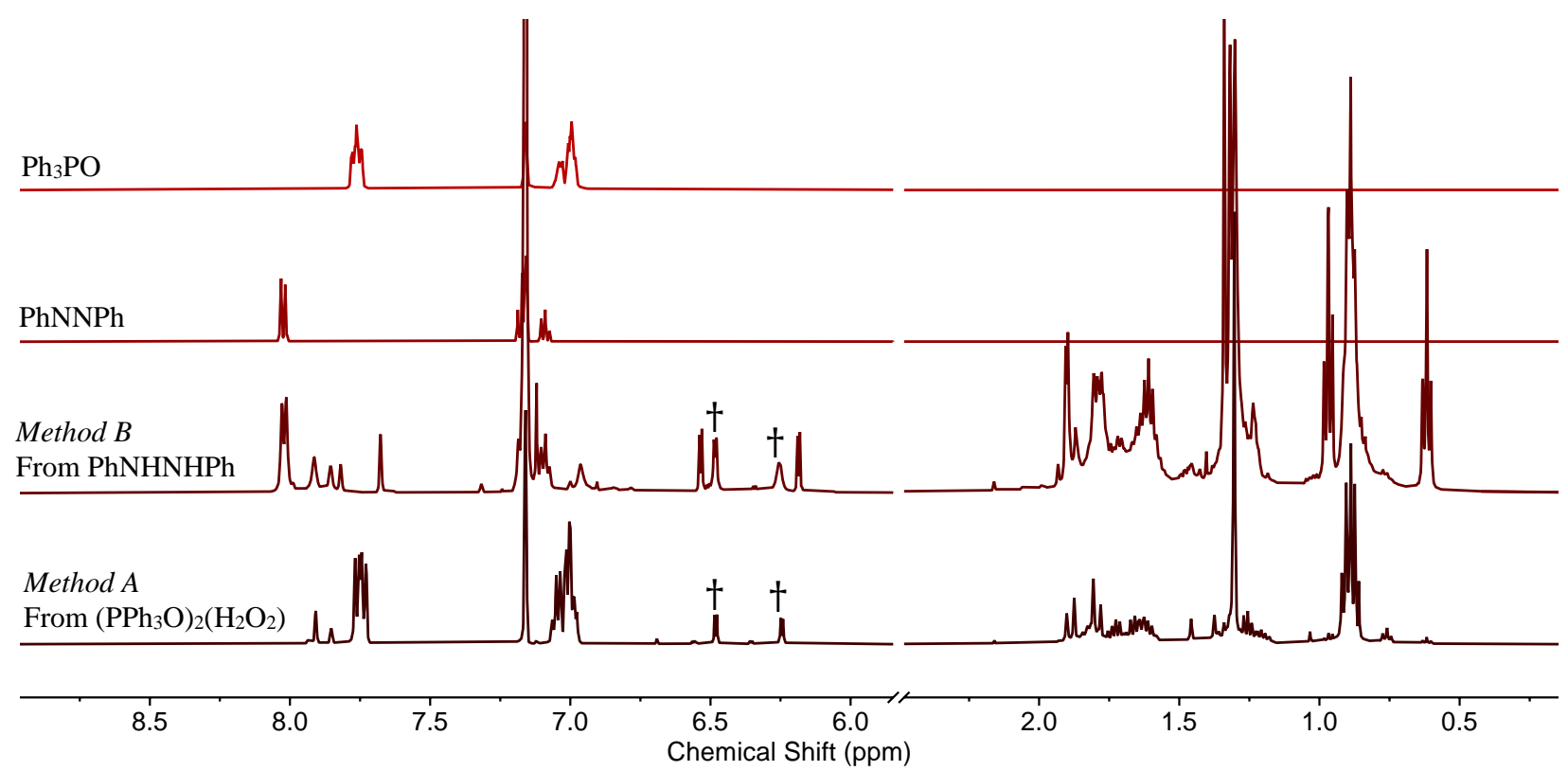

Figure S22. ${ }^{1} \mathrm{H}$ NMR spectra overlay $\left(500 \mathrm{MHz}, \mathrm{C}_{6} \mathrm{D}_{6}\right)$ of $\left({ }^{\mathrm{EMind}} \mathrm{L}\right) \mathrm{Cu}\left(\mathrm{H}_{2} \mathrm{O}_{2}\right)$ (7) prepared by $\left(\mathrm{PPh}_{3} \mathrm{O}\right)_{2}\left(\mathrm{H}_{2} \mathrm{O}_{2}\right)$ (bottom) and by treatment of $\left({ }^{\mathrm{EMind}} \mathrm{L}\right) \mathrm{Cu}\left(\mathrm{O}_{2}\right)(2)$ with $\mathrm{PhNHNHPh}$ (lower middle, unassigned resonances correspond to unreacted 2). Reference azoarene (upper middle) and $\mathrm{PPh}_{3} \mathrm{O}$ (top) are presented as ${ }^{1} \mathrm{H}$ NMR comparison spectra. Obelisk refers to diagnostic dipyrrin $\mathrm{C}-H$ resonances. 


\section{Supporting Spectroscopic Characterization.}
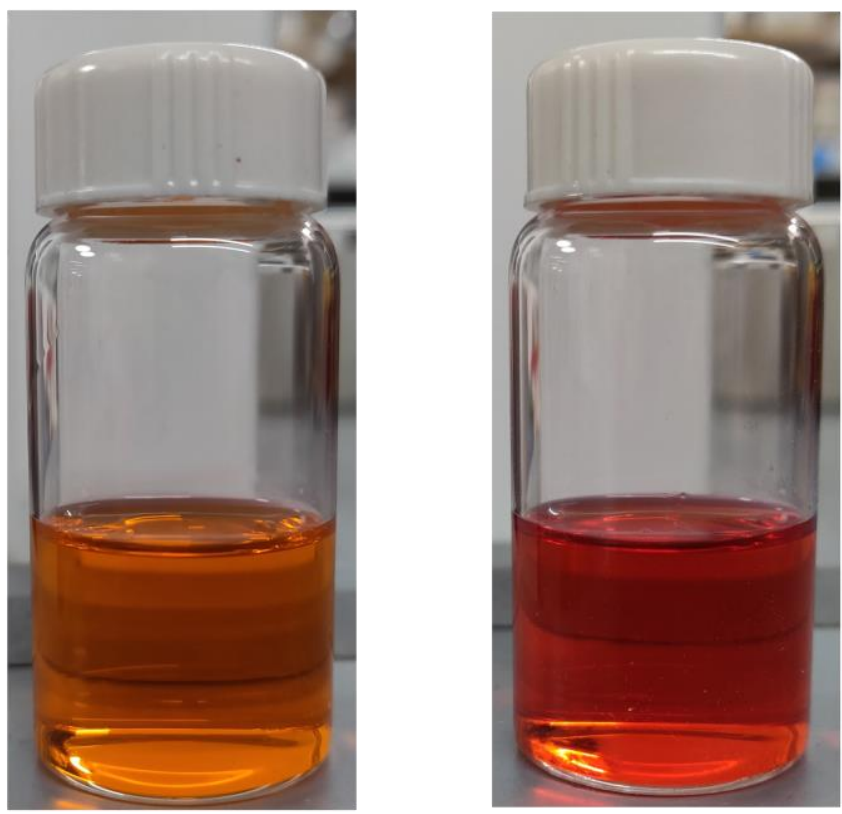

Figure S23. Visual comparison of ( $\left.{ }^{\mathrm{EMind}} \mathrm{L}\right) \mathrm{Cu}\left(\mathrm{N}_{2}\right)(\mathbf{1})$ in hexanes at $20 \mu \mathrm{M}$ concentration (left, orange) and subsequent air exposure and agitation over five seconds to yield $\left({ }^{\mathrm{EMind}} \mathrm{L}\right) \mathrm{Cu}\left(\mathrm{O}_{2}\right)(\mathbf{2})$ in quantitative yield.

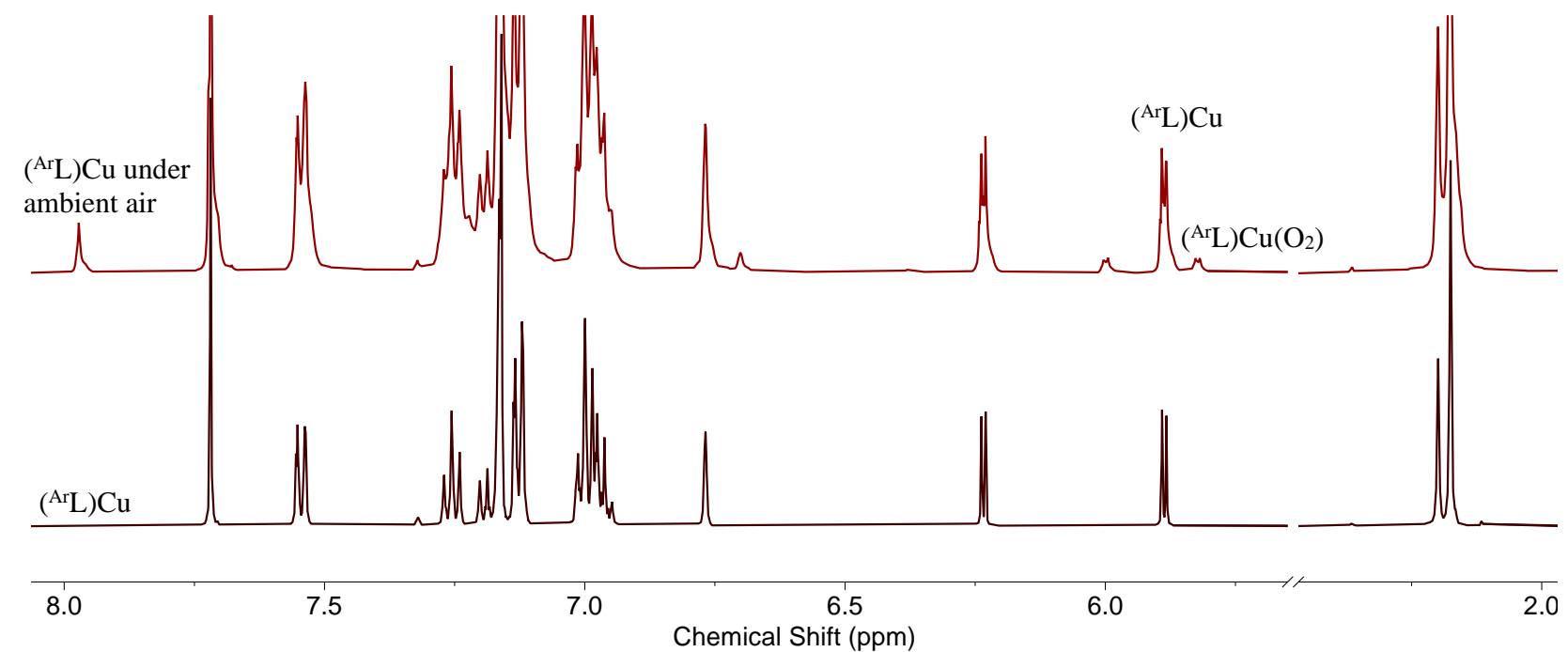

Figure S24. Truncated ${ }^{1} \mathrm{H}$ NMR spectra of $\left(500 \mathrm{MHz}, \mathrm{C}_{6} \mathrm{D}_{6}\right)$ of ( $\left.{ }^{\mathrm{Ar}} \mathrm{L}\right) \mathrm{Cu}$ (bottom, maroon) with subsequent air exposure, yielding growth of $\left({ }^{\mathrm{Ar}} \mathrm{L}\right) \mathrm{Cu}\left(\mathrm{O}_{2}\right)$ within five minutes prior to formation of additional paramagnetic species and $\left({ }^{\mathrm{Ar}} \mathrm{L}\right) \mathrm{H}($ top, red $)$. 


\section{$\mathrm{O}_{2}$ Transfer from ( $\left.{ }^{\mathrm{EMind}} \mathrm{L}\right) \mathrm{Cu}\left(\mathrm{O}_{2}\right)(2)$ to $\mathrm{IMes}_{2} \mathrm{Pd}$.}

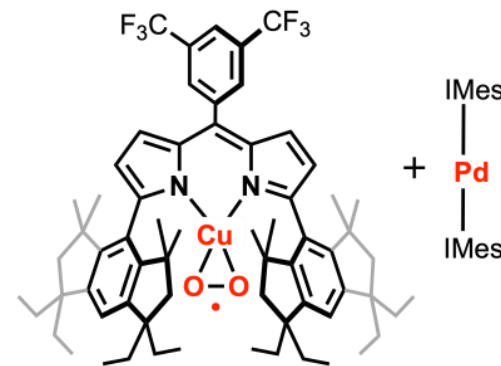

(2)

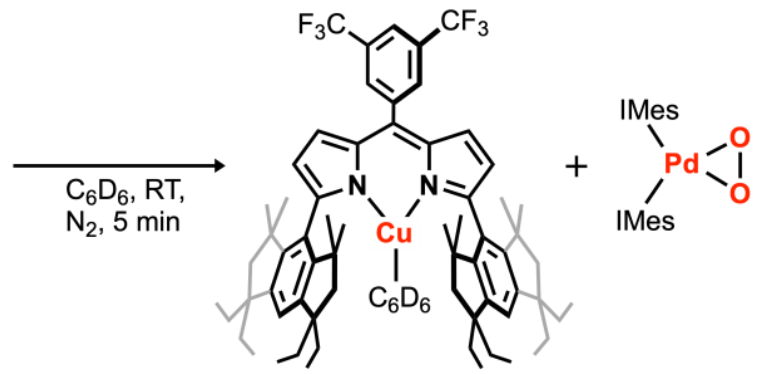

$\left(1-\mathrm{C}_{6} \mathrm{H}_{6}\right)$

General Procedures. In a dinitrogen-filled drybox, a yellow solution ${ }^{3}$ of $\mathrm{IMes}_{2} \mathrm{Pd}(0.005 \mathrm{~g}, 0.007$ mmol, 1.0 equiv.) in $\mathrm{C}_{6} \mathrm{D}_{6}($ ca. $0.5 \mathrm{~mL})$ was added to solid $\left({ }^{\text {EMind }} \mathrm{L}\right) \mathrm{Cu}\left(\mathrm{O}_{2}\right)(2)(0.006 \mathrm{~g}, 0.007$ mmol, 1.0 equiv.), resulting in a Carmine red solution which changed color to orange within five minutes. The solution was then transferred to an NMR tube and checked by multinuclear $\left({ }^{1} \mathrm{H} /{ }^{19} \mathrm{~F}\right)$

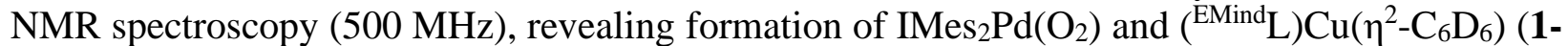
benzene) as the major species. Analysis by ${ }^{19} \mathrm{~F}$ NMR reveals $c a .80 \%$ of ${ }^{19} \mathrm{~F}$ active species as $\mathbf{1 -}$ benzene.

Note $-\mathbf{2}$ should be imported into the drybox immediately prior to the reaction, noting prolonged exposure of 2 to a dinitrogen atmosphere affords trace quantities of $\mathrm{O}_{2}$ dissociation.

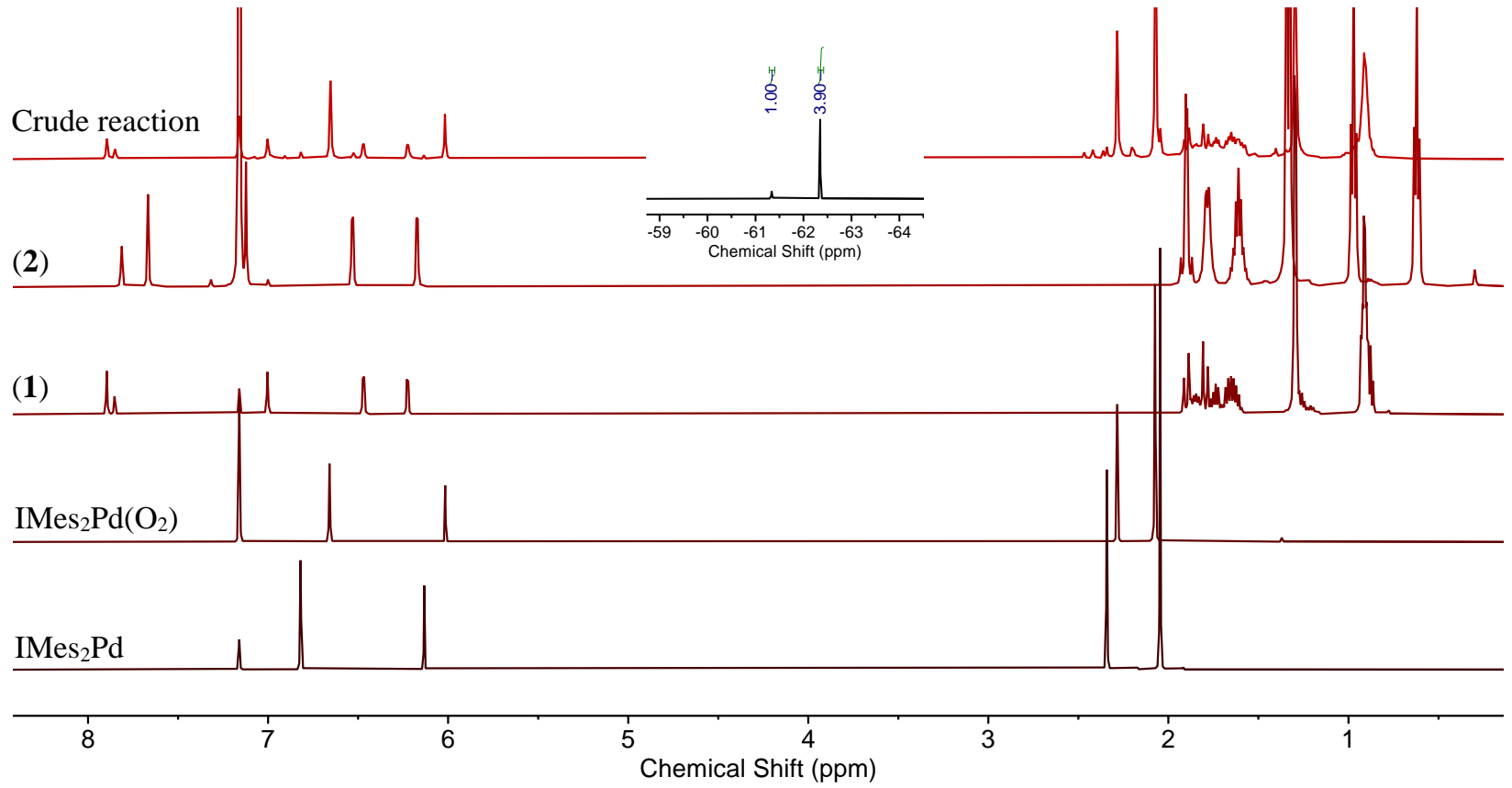

Figure S25. Stacked ${ }^{1} \mathrm{H}$ NMR spectra $\left(500 \mathrm{MHz}, \mathrm{C}_{6} \mathrm{D}_{6}\right)$ of IMes $2 \mathrm{Pd}$ (bottom), IMes $2 \mathrm{Pd}\left(\mathrm{O}_{2}\right)($ lower bottom), ( $\left.{ }^{\mathrm{EMind}} \mathrm{L}\right) \mathrm{Cu}\left(\eta^{2}-\mathrm{C}_{6} \mathrm{D}_{6}\right)$ (1-benzene) (middle), ( $\left.{ }^{\mathrm{EMind}} \mathrm{L}\right) \mathrm{Cu}\left(\mathrm{O}_{2}\right)$ (2) (upper top), and crude reaction mixture (top). A trace quantity of $\mathrm{IMes}_{2} \mathrm{Pd}$ is observed in the crude reaction mixture, attributed to release of $\mathrm{O}_{2}$ from 2 under $\mathrm{N}_{2}$. Inset reveals approximately $80 \%$ of the ${ }^{19} \mathrm{~F} \mathrm{NMR}$ active resonances are attributed to formation of $\mathbf{1}$. 


\section{$\mathrm{O}_{2}$ Displacement through Solvent Exchange.}

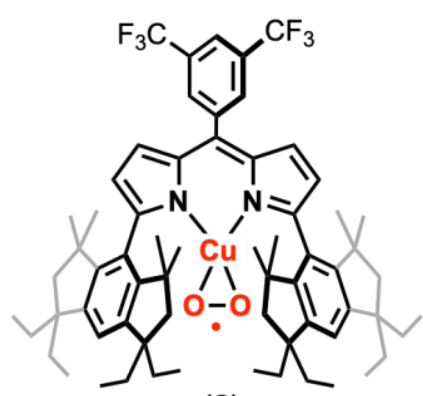

(2)

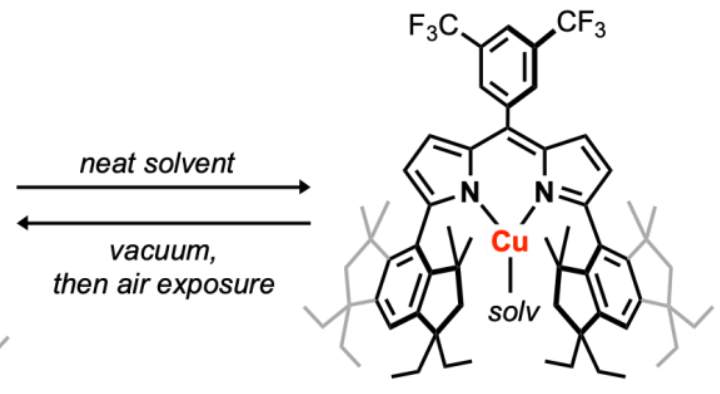

(3 or 4)

General procedure. Under air, $\left({ }^{\mathrm{EMind}} \mathrm{L}\right) \mathrm{Cu}\left(\mathrm{O}_{2}\right)(2)($ ca. $0.010 \mathrm{~g}, 0.010 \mathrm{mmol})$ was dissolved in the specified solvent ( $c a .0 .5 \mathrm{~mL}$ ) and agitated until effervescence ceased. The solution was then transferred to an NMR tube and checked by multinuclear $\left({ }^{1} \mathrm{H} /{ }^{19} \mathrm{~F}\right) \mathrm{NMR}$ spectroscopy $(500 \mathrm{MHz})$. Titration experiments of the specified solvent establish the identity of the new species as the solvent adduct with the secondary species as $\mathbf{2}$, as the ratio of the new species to $\mathbf{2}$ was correlated with increasing equivalents of solvents. The contents of the NMR tube were subsequently transferred to a scintillation vial with removal of solvent in vacuo by rotary evaporation. The contents were subsequently dissolved in $\mathrm{C}_{6} \mathrm{D}_{6}$ and analyzed by multinuclear $\left({ }^{1} \mathrm{H} /{ }^{19} \mathrm{~F}\right) \mathrm{NMR}$ spectroscopy (500 MHz). Assignments were supported by comparison to authentic samples of 2.

Note - all solvents, including $\mathrm{C}_{6} \mathrm{D}_{6}$, were checked by ${ }^{1} \mathrm{H}$ NMR to ensure complete exclusion of $\mathrm{CH}_{2} \mathrm{Cl}_{2}$ as a possible contaminant, noting facile partial formation of ( $\left.{ }^{\mathrm{EMind}} \mathrm{L}\right) \mathrm{Cu}\left(\mathrm{CH}_{2} \mathrm{Cl}_{2}\right)$ (4) in the presence of trace dichloromethane. To avoid possible contamination, the storage container of $\mathrm{C}_{6} \mathrm{D}_{6}$ was only employed in a fume hood strictly devoid of any $\mathrm{CH}_{2} \mathrm{Cl}_{2}$. 
Formation of ( $\left.{ }^{\text {EMind }} \mathrm{L}\right) \mathrm{Cu}\left(\right.$ toluene) (3) from $\left({ }^{\mathrm{EMind}} \mathrm{L}\right) \mathrm{Cu}\left(\mathrm{O}_{2}\right)(2)$.

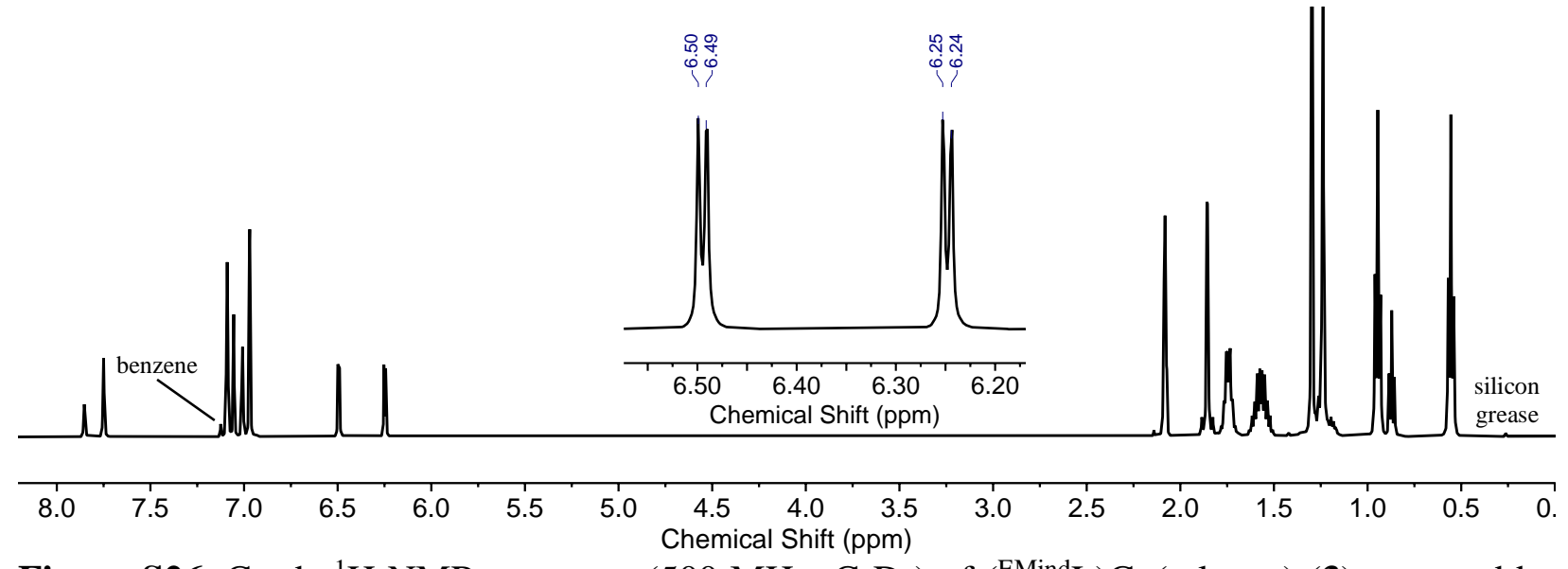

Figure S26. Crude ${ }^{1} \mathrm{H}$ NMR spectrum $\left(500 \mathrm{MHz}, \mathrm{C}_{7} \mathrm{D}_{8}\right)$ of ( $\left.{ }^{\mathrm{EMind}} \mathrm{L}\right) \mathrm{Cu}$ (toluene) (3) prepared by dissolution of $\left({ }^{\text {EMind }} \mathrm{L}\right) \mathrm{Cu}\left(\mathrm{O}_{2}\right)$ (2) in neat $\mathrm{C}_{7} \mathrm{D}_{8}$, depicting formation of a single species. Inset highlights the formation of a single dipyrrin-containing based on the sole set of dipyrrin $\mathrm{C}-H$ resonances.

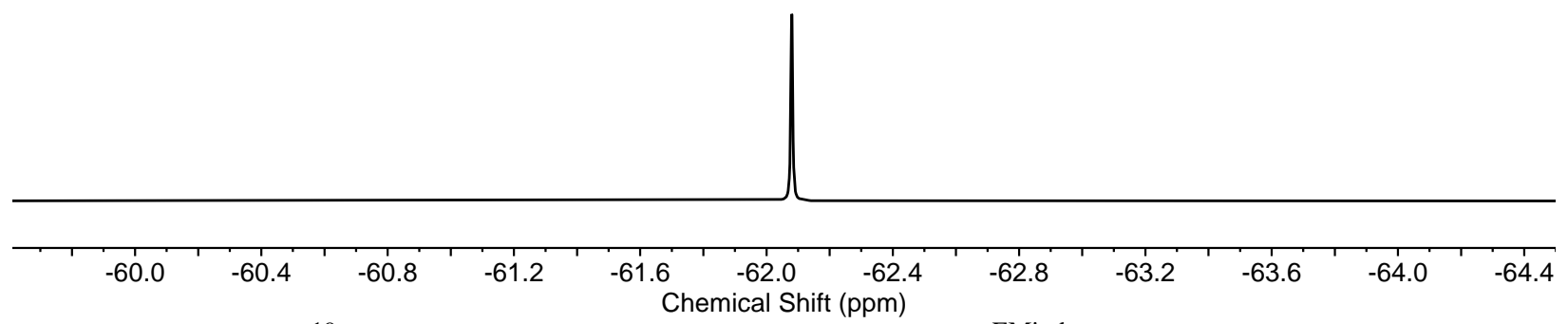

Figure 27. Crude ${ }^{19} \mathrm{~F}$ NMR spectrum $\left(500 \mathrm{MHz}, \mathrm{C}_{7} \mathrm{D}_{8}\right)$ of $\left({ }^{\text {EMind }} \mathrm{L}\right) \mathrm{Cu}$ (toluene) (3) prepared by dissolution of $\left({ }^{\mathrm{EMind}} \mathrm{L}\right) \mathrm{Cu}\left(\mathrm{O}_{2}\right)(2)$ in neat $\mathrm{C}_{7} \mathrm{D}_{8}$, depicting formation of a single species. 
Reformation of $\left({ }^{(E M i n d} \mathrm{L}\right) \mathrm{Cu}\left(\mathrm{O}_{2}\right)(2)$ from $\left({ }^{\mathrm{EMind}} \mathrm{L}\right) \mathrm{Cu}$ (toluene) (3).

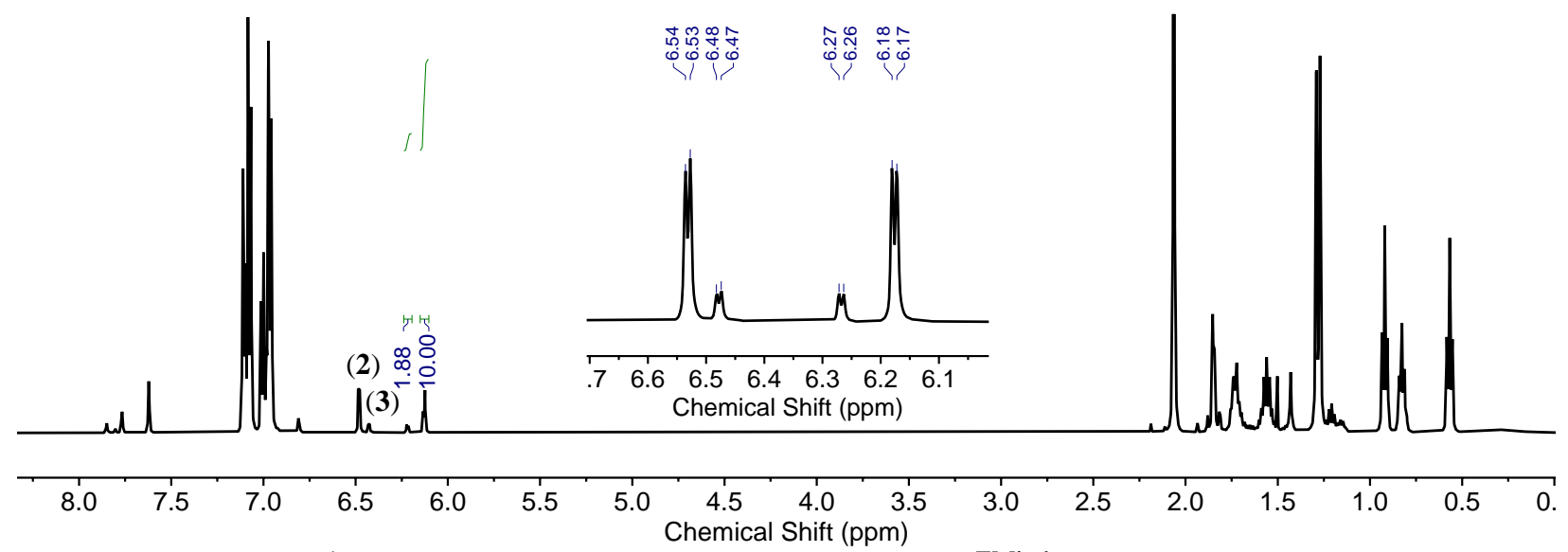

Figure S28. Crude ${ }^{1} \mathrm{H}$ NMR spectrum $\left(500 \mathrm{MHz}, \mathrm{C}_{6} \mathrm{D}_{6}\right)$ of $\left({ }^{\mathrm{EMind}} \mathrm{L}\right) \mathrm{Cu}\left(\mathrm{O}_{2}\right)(2)$ prepared under air by removal of solvent from in situ prepared $\left({ }^{\mathrm{EMind}} \mathrm{L}\right) \mathrm{Cu}$ (toluene) $(\mathbf{3})$. The baseline resonances denote unconverted 3 ( $\mathrm{ca}$. $16 \%$ by integration). Inset highlights two sets of dipyrrin $\mathrm{C}-\mathrm{H}$ resonances.

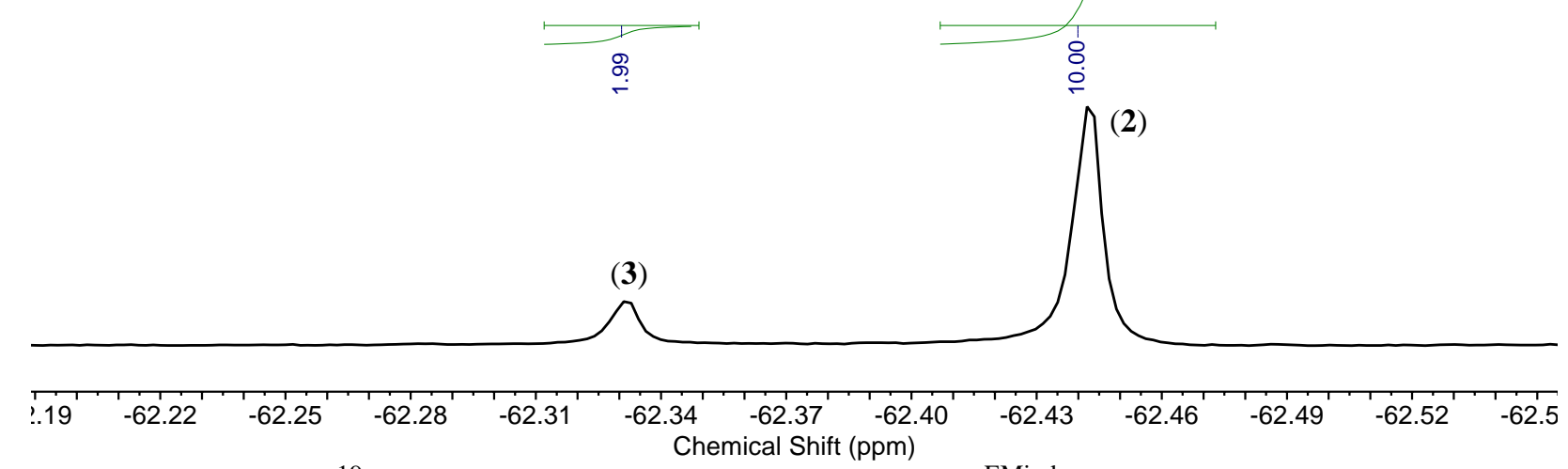

Figure S29. Crude ${ }^{19} \mathrm{~F}$ NMR spectrum $\left(470 \mathrm{MHz}, \mathrm{C}_{6} \mathrm{D}_{6}\right)$ of $\left({ }^{(\mathrm{EMind}} \mathrm{L}\right) \mathrm{Cu}\left(\mathrm{O}_{2}\right)(2)$ prepared under air by removal of solvent from in situ prepared $\left({ }^{\mathrm{EMind}} \mathrm{L}\right) \mathrm{Cu}$ (toluene) $(\mathbf{3})$. The baseline resonances denote unconverted $\mathbf{3}$ ( $c a .16 \%$ by integration). 
Formation of ( $\left.{ }^{\mathrm{EMind}} \mathrm{L}\right) \mathrm{Cu}\left(\mathrm{CH}_{2} \mathrm{Cl}_{2}\right)(4)$ from $\left({ }^{\mathrm{EMind}} \mathrm{L}\right) \mathrm{Cu}\left(\mathrm{O}_{2}\right)(2)$.

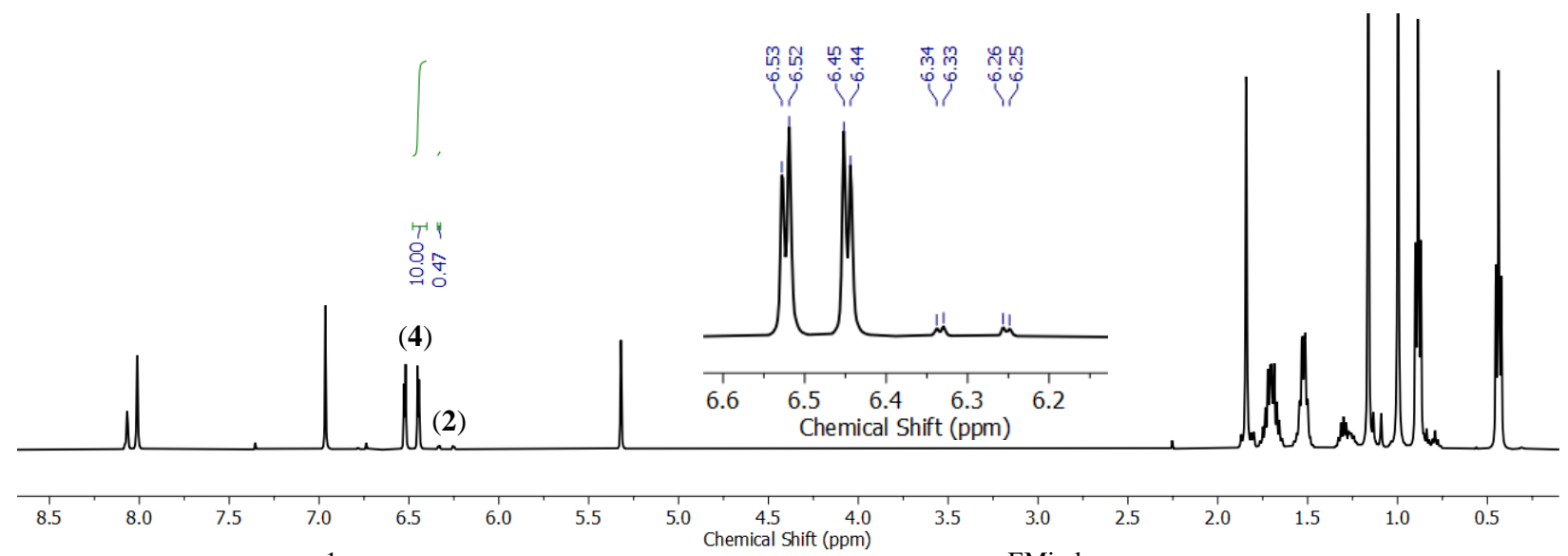

Figure S30. Crude ${ }^{1} \mathrm{H}$ NMR spectrum $\left(500 \mathrm{MHz}, \mathrm{CD}_{2} \mathrm{Cl}_{2}\right)$ of ( $\left.{ }^{\mathrm{EMind}} \mathrm{L}\right) \mathrm{Cu}\left(\mathrm{CH}_{2} \mathrm{Cl}_{2}\right)$ (4) prepared by dissolution of $\left({ }^{\mathrm{EMind}} \mathrm{L}\right) \mathrm{Cu}\left(\mathrm{O}_{2}\right)(2)$ in neat $\mathrm{CD}_{2} \mathrm{Cl}_{2}$. The baseline resonances denote unconverted 2 (ca. $5 \%$ by integration). Inset highlights two sets of dipyrrin $\mathrm{C}-H$ resonances.

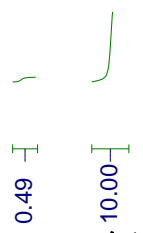

(2)

\begin{tabular}{llllllllllll}
\hline-60.2 & -60.4 & -60.6 & -60.8 & -61.0 & -61.2 & -61.4 & -61.6 & -61.8 & -62.0 & -62.2 & -62.4 \\
Chemical Shift (ppm)
\end{tabular}

Figure S31. Crude ${ }^{19} \mathrm{~F}$ NMR spectrum $\left(470 \mathrm{MHz}, \mathrm{CD}_{2} \mathrm{Cl}_{2}\right)$ of $\left({ }^{\mathrm{EMind}} \mathrm{L}\right) \mathrm{Cu}\left(\mathrm{CH}_{2} \mathrm{Cl}_{2}\right)(4)$ prepared by dissolution of $\left({ }^{\mathrm{EMind}} \mathrm{L}\right) \mathrm{Cu}\left(\mathrm{O}_{2}\right)(\mathbf{2})$ in neat $\mathrm{CD}_{2} \mathrm{Cl}_{2}$. The baseline resonances denote unconverted 2 (ca. $5 \%$ by integration). 
Reformation of $\left({ }^{\mathrm{EMind}} \mathrm{L}\right) \mathrm{Cu}\left(\mathrm{O}_{2}\right)(2)$ from $\left({ }^{\mathrm{EMind}} \mathrm{L}\right) \mathrm{Cu}\left(\mathrm{CH}_{2} \mathrm{Cl}_{2}\right)(4)$.

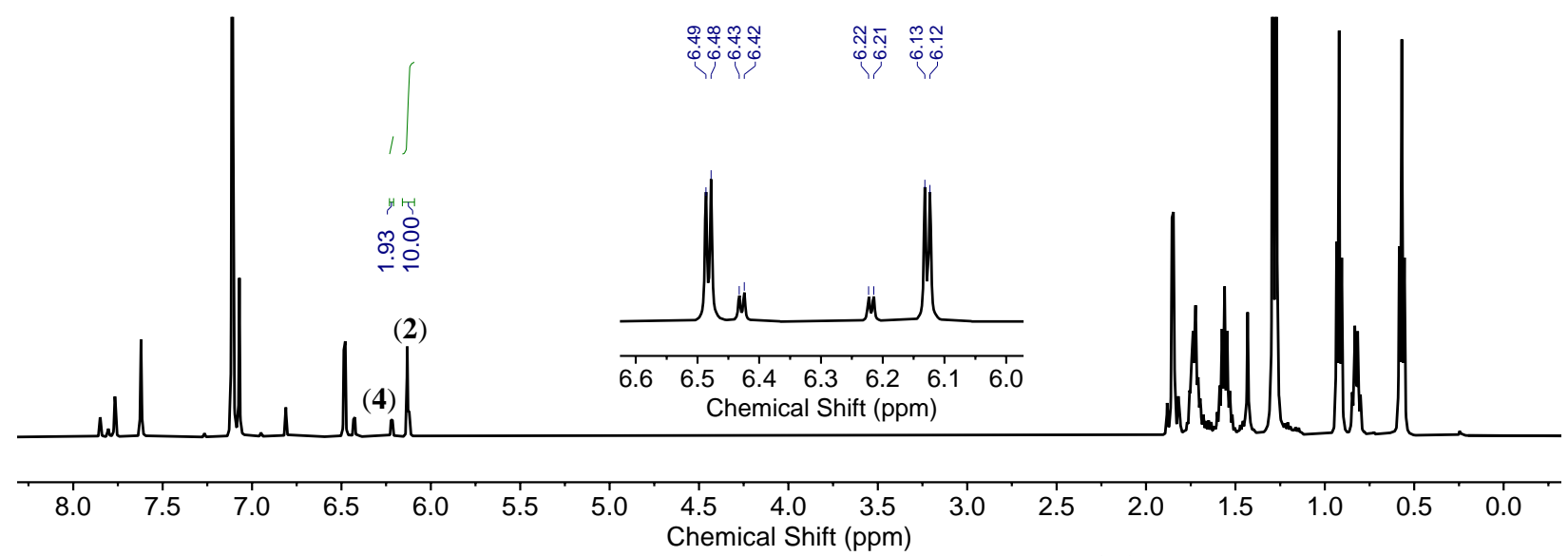

Figure S32. Crude ${ }^{1} \mathrm{H}$ NMR spectrum $\left(500 \mathrm{MHz}, \mathrm{C}_{6} \mathrm{D}_{6}\right)$ of $\left({ }^{\mathrm{EMind}} \mathrm{L}\right) \mathrm{Cu}\left(\mathrm{O}_{2}\right)$ (2) prepared by removal of solvent from in situ prepared $\left({ }^{\mathrm{EMind}} \mathrm{L}\right) \mathrm{Cu}\left(\mathrm{CH}_{2} \mathrm{Cl}_{2}\right)$ (4) under air. The baseline resonances denote unconverted 4 ( $c a .16 \%$ by integration). Inset highlights two sets of dipyrrin $\mathrm{C}-H$ resonances.

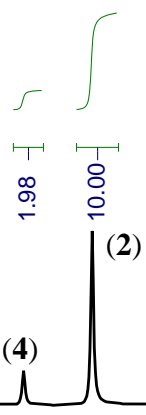

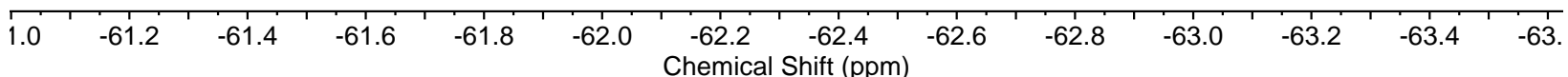

Figure S33. Crude ${ }^{19} \mathrm{~F}$ NMR spectrum $\left(470 \mathrm{MHz}, \mathrm{C}_{6} \mathrm{D}_{6}\right)$ of $\left({ }^{\mathrm{EMind}} \mathrm{L}\right) \mathrm{Cu}\left(\mathrm{O}_{2}\right)(2)$ prepared under air by removal of solvent from in situ prepared $\left({ }^{\mathrm{EMind}} \mathrm{L}\right) \mathrm{Cu}\left(\mathrm{CH}_{2} \mathrm{Cl}_{2}\right)(\mathbf{4})$. The baseline resonances denote unconverted 4 ( $c a .17 \%$ by integration). 
Table S1. Summary of $\mathrm{O}_{2}$ Displacement from Reagent Addition to ( $\left.{ }^{\mathrm{EMind}} \mathrm{L}\right) \mathrm{Cu}\left(\mathrm{O}_{2}\right)(\mathbf{2})$.

\begin{tabular}{|c|c|c|c|}
\hline Ligand Identity & Observation & $\% 2$ after addition & $\% 2$ after evacuation \\
\hline Alkanes $^{\mathrm{a}}$ & No $\mathrm{O}_{2}$ Displacement & 100 & 100 \\
\hline Methanol $^{\mathrm{a}}$ & No $\mathrm{O}_{2}$ Displacement & 100 & 100 \\
\hline Benzene $^{\mathrm{a}}$ & $\mathrm{No} \mathrm{O}_{2}$ Displacement & 100 & 100 \\
\hline Diethyl Ether ${ }^{\mathrm{a}}$ & $\mathrm{No} \mathrm{O}_{2}$ Displacement & 100 & 100 \\
\hline Dioxane $^{\mathrm{a}}$ & No $\mathrm{O}_{2}$ Displacement & 100 & 100 \\
\hline Hydrogen Gas ${ }^{\mathrm{d}}$ & $\mathrm{No} \mathrm{O}_{2}$ Displacement & 100 & 100 \\
\hline Toluene $^{\mathrm{a}}$ & Reversible $\mathrm{O}_{2}$ Displacement & 0 & 84 \\
\hline Dichloromethane $^{a}$ & Reversible $\mathrm{O}_{2}$ Displacement & 5 & 83 \\
\hline Carbon Disulfide ${ }^{a}$ & Reversible $\mathrm{O}_{2}$ Displacement & 15 & 80 \\
\hline Cyclohexene $\mathrm{e}^{\mathrm{b}}$ & Reversible $\mathrm{O}_{2}$ Displacement & 70 & 100 \\
\hline Thiophene $^{\mathrm{b}}$ & Reversible $\mathrm{O}_{2}$ Displacement & 2 & 75 \\
\hline Hydroxylamine $^{\mathrm{b}}$ & Reversible $\mathrm{O}_{2}$ Displacement ${ }^{\mathrm{e}}$ & 0 & 100 \\
\hline Chlorobenzene $^{\mathrm{b}}$ & Partial $\mathrm{O}_{2}$ Displacement & 85 & \lrcorner$^{f}$ \\
\hline 1,2-Dichlorobenzene ${ }^{\mathrm{b}}$ & Partial $\mathrm{O}_{2}$ Displacement & 82 & $f$ \\
\hline Acetone $^{\mathrm{a}}$ & Partial $\mathrm{O}_{2}$ Displacement & 80 & $f$ \\
\hline Dimethylsulfoxide $^{\mathrm{b}}$ & Partial $\mathrm{O}_{2}$ Displacement & 30 & $f^{f}$ \\
\hline Pyrazine $^{c}$ & Irreversible $\mathrm{O}_{2}$ Displacement & 0 & 0 \\
\hline Hydrazine $^{c}$ & Irreversible $\mathrm{O}_{2}$ Displacement & 0 & 0 \\
\hline Acetonitrile $^{\mathrm{b}}$ & Irreversible $\mathrm{O}_{2}$ Displacement & 0 & 0 \\
\hline Carbon Monoxide $^{\mathrm{d}}$ & Irreversible $\mathrm{O}_{2}$ Displacement & 0 & 0 \\
\hline Dimethylsulfide $^{c}$ & Irreversible $\mathrm{O}_{2}$ Displacement & 0 & 0 \\
\hline Chloroform $^{\mathrm{a}}$ & Oxidation to $\left({ }^{\text {EMind }} \mathrm{L}\right) \mathrm{CuCl}$ & 0 & $-{ }^{\mathrm{e}}$ \\
\hline Carbon Tetrachloride $^{\mathrm{a}}$ & Oxidation to ( $\left.{ }^{\text {EMind }} \mathrm{L}\right) \mathrm{CuCl}$ & 0 & $-{ }^{e}$ \\
\hline Nitromethane ${ }^{\mathrm{b}}$ & Partial Demetallation & 85 & $-^{\mathrm{e}}$ \\
\hline 1,1-Dichloroethane ${ }^{\mathrm{b}}$ & Partial Demetallation & 80 & $-^{\mathrm{e}}$ \\
\hline Tetrahydrofuran ${ }^{\mathrm{a}}$ & Decomposition upon vacuum & 45 & $-^{\mathrm{e}}$ \\
\hline Tetrachloroethylene $^{c}$ & Decomposition upon vacuum & 70 & $-^{\mathrm{e}}$ \\
\hline Ammonia $^{\mathrm{b}}$ & Complete Demetallation & 0 & $-^{\mathrm{e}}$ \\
\hline
\end{tabular}

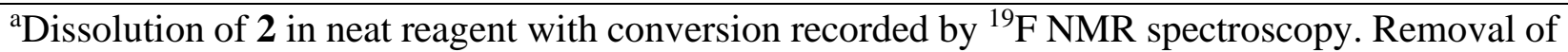
solvent followed by dissolution in $\mathrm{C}_{6} \mathrm{D}_{6}$ was employed to determine regeneration of 2 . ${ }^{\mathrm{b}} 100$ equiv. of reagent employed to 2 in $\mathrm{C}_{6} \mathrm{D}_{6}$. ${ }^{\mathrm{c}} \mathrm{S}$ tochiometric reagent added to 2 in $\mathrm{C}_{6} \mathrm{D}_{6} \cdot{ }^{\mathrm{d}} 1 \mathrm{~atm}$ added to 2 in

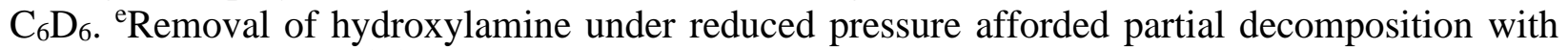
formation of $\mathbf{2}$ and ( $\left.{ }^{\mathrm{EMind}} \mathrm{L}\right) \mathrm{H}$. Nonetheless, removal of hydroxylamine by slow evaporation under air under ambient conditions reafforded $\mathbf{2}$ in quantitative yield. ${ }^{\mathrm{f}} \mathrm{Not}$ measured due to decomposition to low conversion or inability to remove excess ligating reagent. 
Reformation of ( $\left.{ }^{\mathrm{EMind}} \mathrm{L}\right) \mathrm{Cu}\left(\mathrm{O}_{2}\right)$ (2) from ( $\left.{ }^{\mathrm{MMind}} \mathrm{L}\right) \mathrm{CuI}(6)$.

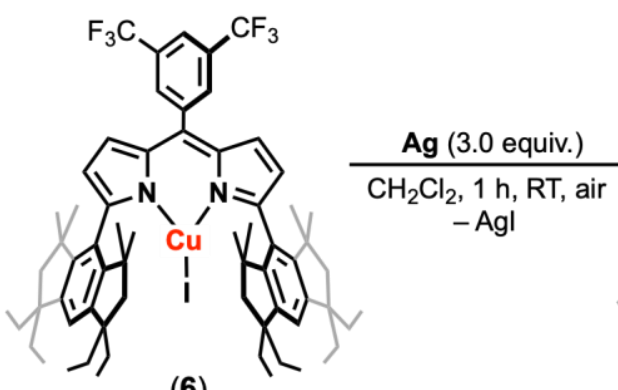

(6)

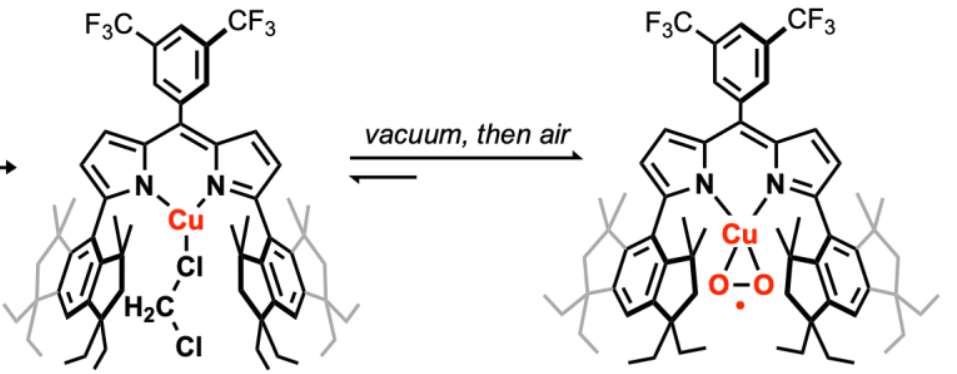

(4)

(2)

General Procedure. Treatment of $\left({ }^{\mathrm{EMind}} \mathrm{L}\right) \mathrm{CuI}(\mathbf{6})$ with metallic silver in dichloromethane under air afforded $\left({ }^{\text {EMind }} \mathrm{L}\right) \mathrm{Cu}\left(\mathrm{CH}_{2} \mathrm{Cl}_{2}\right)$ (4), which could be converted into a mixture of $\mathbf{5}$ and $\left({ }^{\mathrm{EMind}} \mathrm{L}\right) \mathrm{Cu}\left(\mathrm{O}_{2}\right)(2)$ upon removal of excess dichloromethane.

Note I: Chemical reduction of ( $\left.{ }^{\mathrm{EMind}} \mathrm{L}\right) \mathrm{CuI}(\mathbf{6})$ with stoichiometric potassium graphite $\left(\mathrm{KC}_{8}\right)$ under $\mathrm{N}_{2}$ in diethyl ether was able to afford ( $\left.{ }^{\mathrm{EMind}} \mathrm{L}\right) \mathrm{Cu}\left(\mathrm{N}_{2}\right)(\mathbf{1})$ in quantitative yield following workup, which could be converted to 2 upon air exposure. Due to the necessity to conduct the reduction under $\mathrm{N}_{2}$ in this protocol based on the air-sensitivity of $\mathrm{KC}_{8}$, the preparation of 2 through metallic silver under air is favored. 


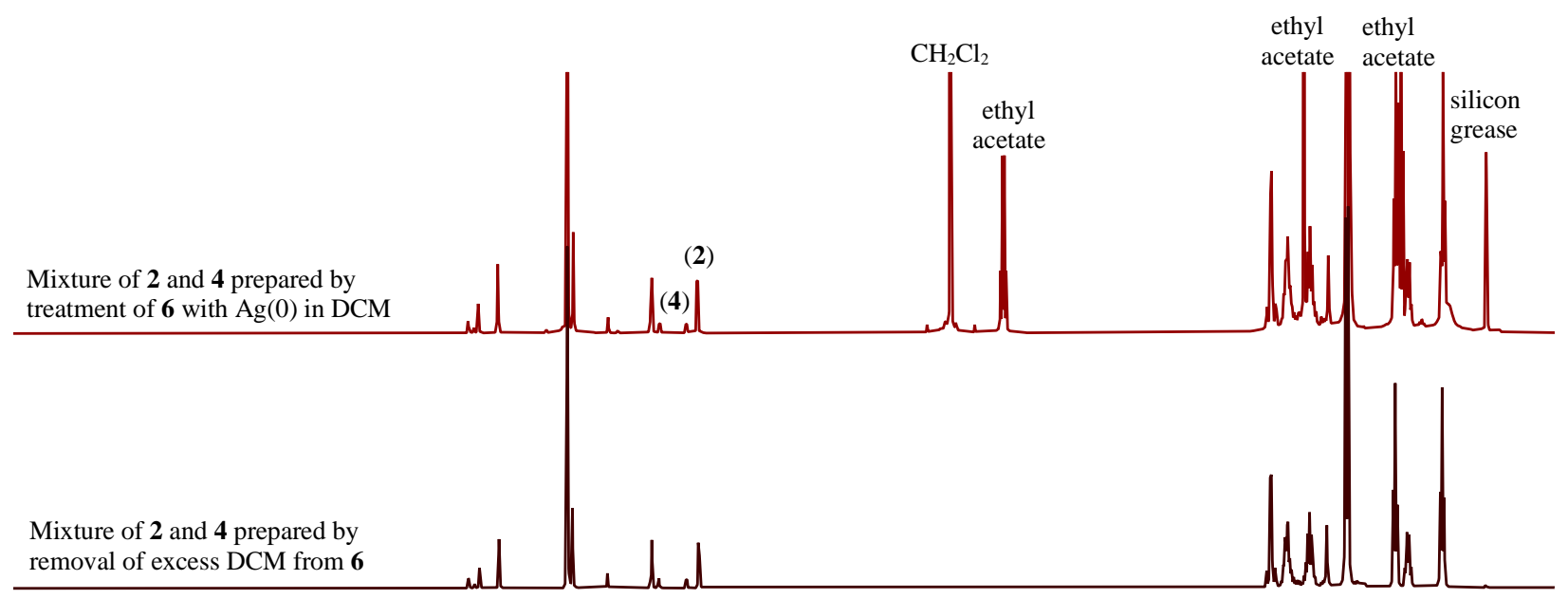

$\begin{array}{lllllllllllllllllllllllllll}11.0 & 10.5 & 10.0 & 9.5 & 9.0 & 8.5 & 8.0 & 7.5 & 7.0 & 6.5 & 6.0 & 5.5 & 5.0 & 4.5 & 4.0 & 3.5 & 3.0 & 2.5 & 2.0 & 1.5 & 1.0 & 0.5 & 0.0\end{array}$

Figure S34. Comparison of ${ }^{1} \mathrm{H}$ NMR spectra $\left(500 \mathrm{MHz}, \mathrm{C}_{6} \mathrm{D}_{6}\right)$ from chemical reduction of ( $\left.{ }^{\text {EMind }} \mathrm{L}\right) \mathrm{CuI}(\mathbf{6})$ under air with metallic silver in dichloromethane $(1 \mathrm{~h}$; top, red $)$ to a mixture of ( $\left.{ }^{\mathrm{EMind}} \mathrm{L}\right) \mathrm{Cu}\left(\mathrm{O}_{2}\right)(2)$ and $\left({ }^{\mathrm{EMind}} \mathrm{L}\right) \mathrm{Cu}\left(\mathrm{CH}_{2} \mathrm{Cl}_{2}\right)(\mathbf{4})$, (bottom, maroon).

Figure S35. Comparison of ${ }^{19} \mathrm{~F}$ NMR spectra $\left(470 \mathrm{MHz}, \mathrm{C}_{6} \mathrm{D}_{6}\right)$ from chemical reduction of ( $\left.{ }^{\text {EMind }} \mathrm{L}\right) \mathrm{CuI}(\mathbf{6})$ under air with metallic silver in dichloromethane $(1 \mathrm{~h}$; top, red $)$ to a mixture of ( $\left.{ }^{\mathrm{EMind}} \mathrm{L}\right) \mathrm{Cu}\left(\mathrm{O}_{2}\right)(2)$ and $\left({ }^{\mathrm{EMind}} \mathrm{L}\right) \mathrm{Cu}\left(\mathrm{CH}_{2} \mathrm{Cl}_{2}\right)(4)$, (bottom, maroon). 


\section{Thermal Cycling between ( $\left.{ }^{\mathrm{EMind}} \mathrm{L}\right) \mathrm{Cu}\left(\mathbf{N}_{2}\right)(1)$ and $\left({ }^{\mathrm{EMind}} \mathrm{L}\right) \mathrm{Cu}\left(\mathrm{O}_{2}\right)(2)$.}

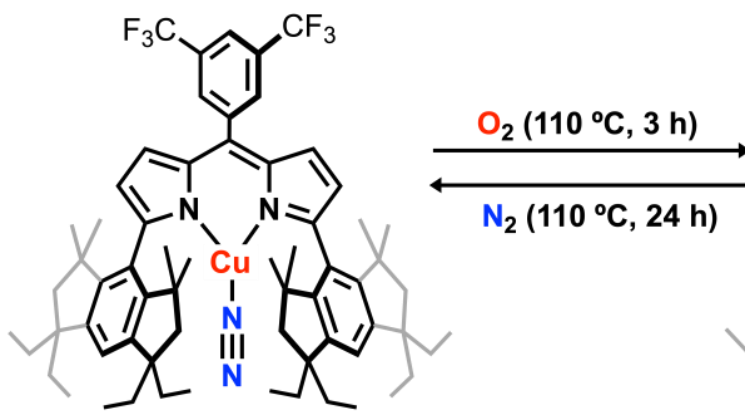

(1)

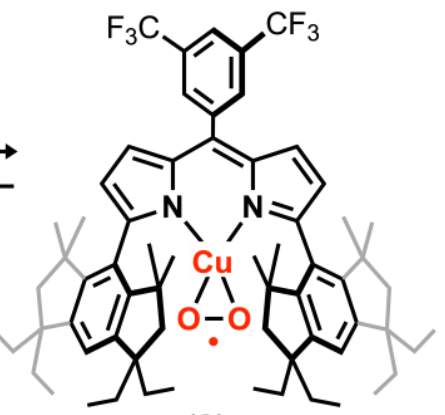

(2)

General Procedures. Under air, crystalline $\left({ }^{\mathrm{EMind}} \mathrm{L}\right) \mathrm{Cu}\left(\mathrm{O}_{2}\right)(2)$ was crushed with a Teflon rod until no additional crystalline facets were apparent and the sample became a finely divided powder, noting the employment of large crystals of 2 results in slow apparent rates of gas exchange. The sample ( $\mathrm{ca} .10 \mathrm{mg}$ ) was loaded into a pre-weighed metal and loaded into a thermogravimetric analysis instrument (TGA500, TA Instruments). The initial mass was recorded, and a temperature ramp of $1{ }^{\circ} \mathrm{C} / \mathrm{min}$ to $110^{\circ} \mathrm{C}$ was set under active $\mathrm{O}_{2}$ flow, followed by holding at this temperature for $24 \mathrm{~h}$ to ensure removal of lattice solvent. The gas stream was switched to $\mathrm{N}_{2}$, and the sample was held at $110^{\circ} \mathrm{C}$ under $\mathrm{N}_{2}$ flow for $24 \mathrm{~h}$. The gas inlet stream was switched to $\mathrm{O}_{2}$, and the sample was held at either $110^{\circ} \mathrm{C}$ (Figure S36) or $30^{\circ} \mathrm{C}$ (Figure S37) under $\mathrm{O}_{2}$ flow for $3 \mathrm{~h}$. During gas switching, an artifact is present by TGA due to the differences in buoyancy of the inlet gas stream (see figure) in which switching from $\mathrm{N}_{2}$ to $\mathrm{O}_{2}$ results in an artifact mass increase, and switching from $\mathrm{O}_{2}$ to $\mathrm{N}_{2}$ results in an artifact mass decrease. This cycling procedure was repeated for a total of five cycles. Once the instrument temperature was set back to room temperature, the sample was subsequently removed from the instrument and analyzed by multinuclear NMR spectroscopy to assess for decomposition.

The non-quantitative conversion of $\mathbf{2}$ into $\mathbf{1}$ is attributed to slow diffusion of $\mathrm{N}_{2}$ into the bulk microcrystalline sample.

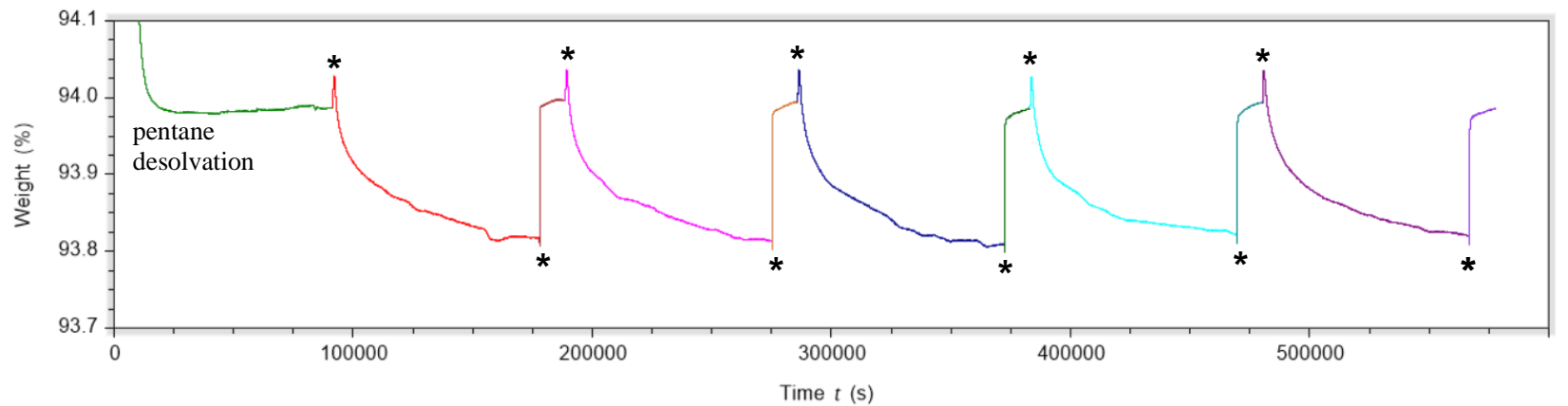

Figurě S36. Uncorrected thermal gravimetric analysis ('IGA) cycling of ( $\left.{ }^{\text {Livinu }} \mathrm{L}\right) \mathrm{C}\left(\mathrm{u}\left(\mathrm{O}_{2}\right)(2)\right.$ under alternating $\mathrm{N}_{2} / \mathrm{O}_{2}$ cycles at a constant temperature of $110^{\circ} \mathrm{C}$. The initial mass loss prior to cycling is attributed to loss of a pentane molecule from the crystalline lattice (theoretical mass loss: 6.18 $\%$, observed $6.02 \%$; difference is attributed to partial desorption of the pentane). Features marked with asterisks are instrument artifacts attributed to switching gas inlet streams. 


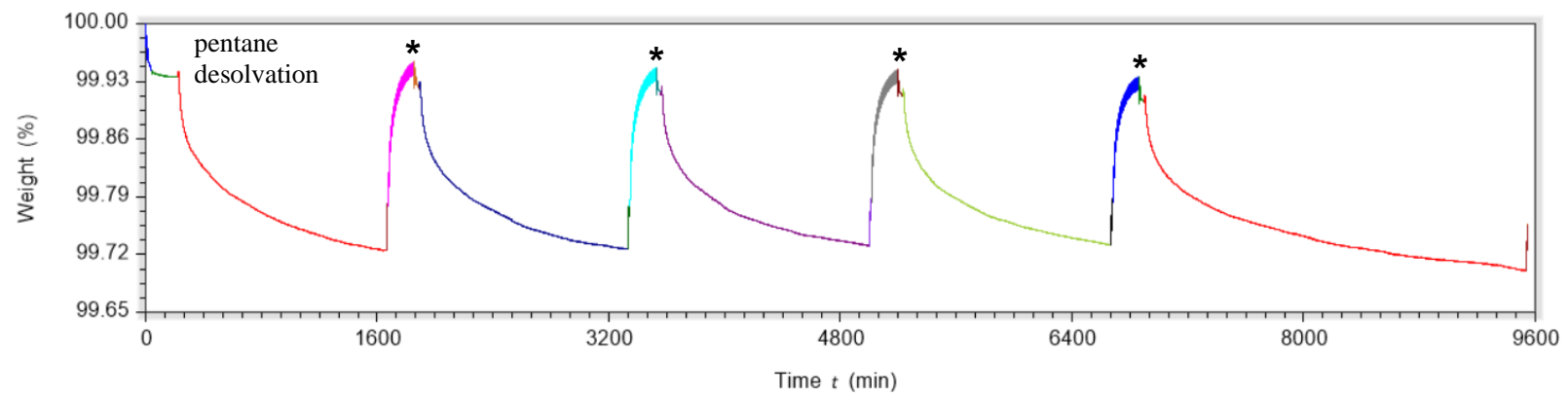

Figure S37. Uncorrected thermal gravimetric analysis (TGA) cycling of ( $\left.{ }^{\mathrm{EMind}} \mathrm{L}\right) \mathrm{Cu}\left(\mathrm{O}_{2}\right)(2)$ under alternating $\mathrm{N}_{2}\left(110^{\circ} \mathrm{C}\right) / \mathrm{O}_{2}\left(30^{\circ} \mathrm{C}\right)$ cycles. Pre-activation of the sample by heating at $100{ }^{\circ} \mathrm{C}$ under vacuum was conducted to remove the bulk of lattice solvent. Features marked with asterisks are instrument artifacts due to thermal pulses at $30^{\circ} \mathrm{C}$.

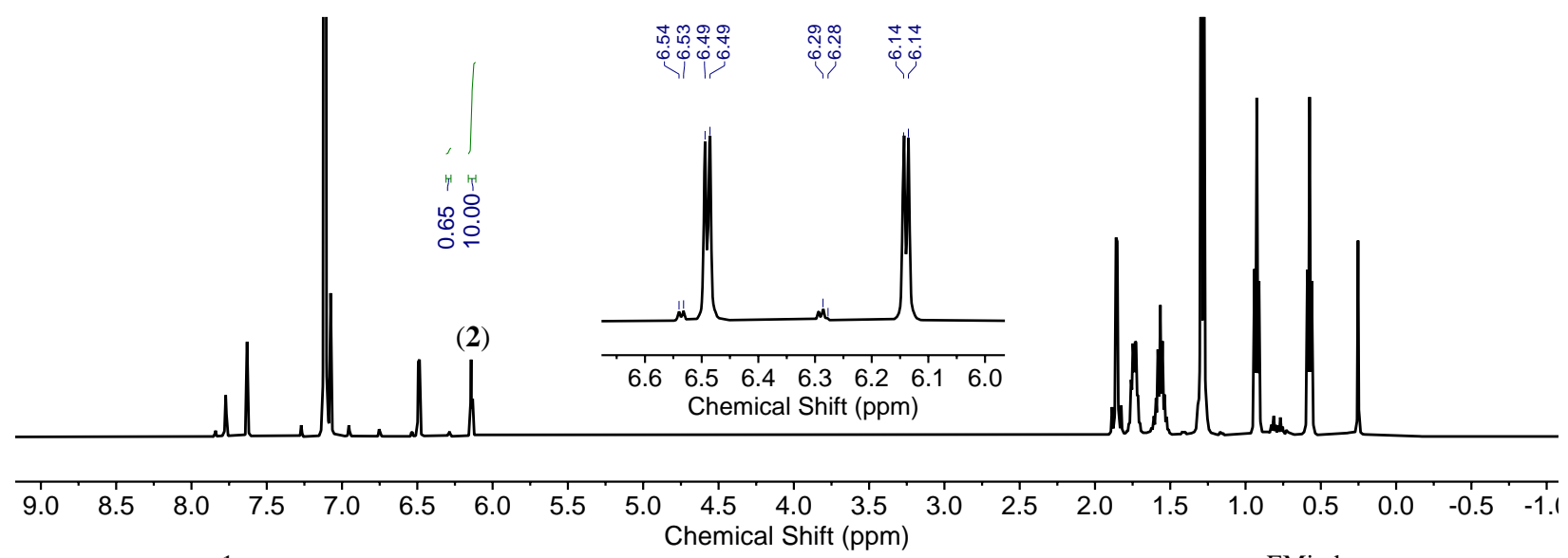

Figure S38. ${ }^{1} \mathrm{H}$ NMR spectra $\left(500 \mathrm{MHz}, \mathrm{C}_{6} \mathrm{D}_{6}\right)$ post-TGA cycling studies of $\left({ }^{\mathrm{EMind}} \mathrm{L}\right) \mathrm{Cu}\left(\mathrm{O}_{2}\right)(\mathbf{2})$, demonstrating minor formation of a diamagnetic decomposition species (ca. $5 \%$ yield) with 2 as the dominant species. Inset highlights dipyrrin $\mathrm{C}-H$ resonances, illustrating minor presence of a secondary species.

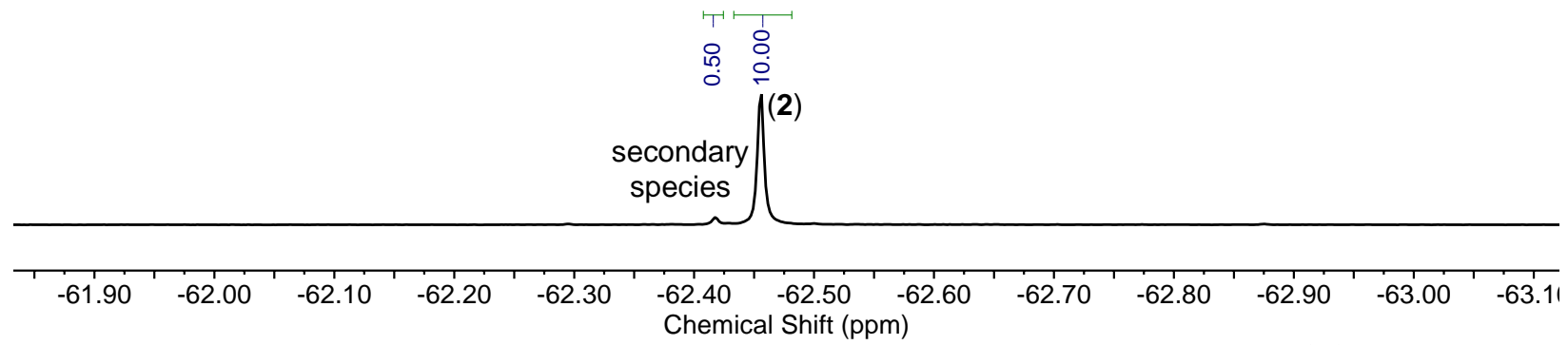

Figure S39. ${ }^{19} \mathrm{~F}$ NMR spectra $\left(470 \mathrm{MHz}, \mathrm{C}_{6} \mathrm{D}_{6}\right)$ post-TGA cycling studies of $\left({ }^{\mathrm{EMind}} \mathrm{L}\right) \mathrm{Cu}\left(\mathrm{O}_{2}\right)(\mathbf{2})$, demonstrating minor formation of a diamagnetic decomposition species (ca. $5 \%$ yield) as identified independently by ${ }^{1} \mathrm{H}$ NMR spectroscopy. 


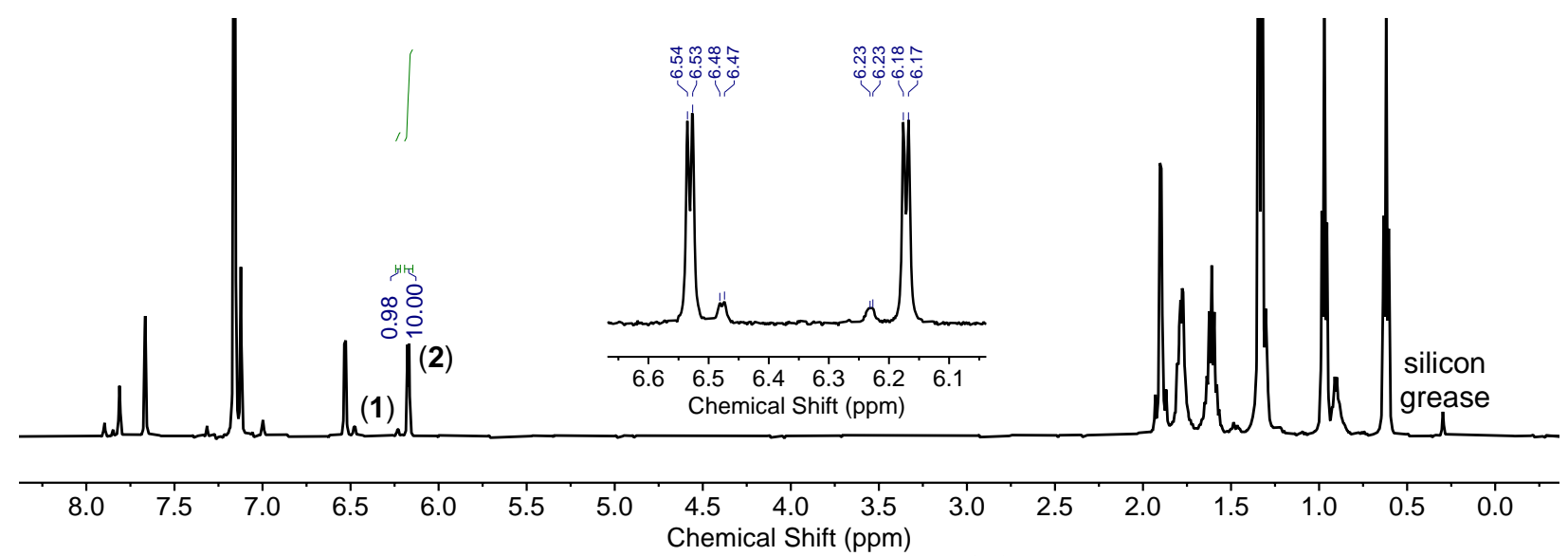

Figure S40. ${ }^{1} \mathrm{H}$ NMR spectra $\left(500 \mathrm{MHz}, \mathrm{C}_{6} \mathrm{D}_{6}\right)$ of $\left({ }^{\mathrm{EMind}} \mathrm{L}\right) \mathrm{Cu}\left(\mathrm{O}_{2}\right)$ (2) under $\mathrm{N}_{2}$, showing equilibration with $\mathrm{N}_{2}$ to yield $\left({ }^{\mathrm{EMind}} \mathrm{L}\right) \mathrm{Cu}\left(\mathrm{O}_{2}\right)$ (1) in $c a .9 \%$ yield. Inset depicts dipyrrin $\mathrm{C}-\mathrm{H}$ resonances for $\mathbf{1}$ and $\mathbf{2}$.

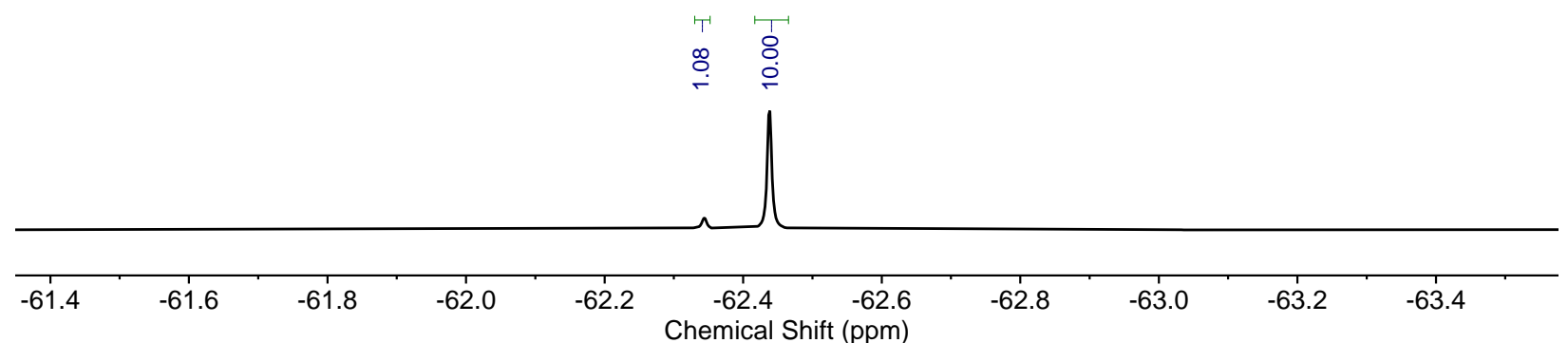

Figure S41. ${ }^{19} \mathrm{~F}$ NMR spectra $\left(470 \mathrm{MHz}, \mathrm{C}_{6} \mathrm{D}_{6}\right)$ of $\left({ }^{\mathrm{EMind}} \mathrm{L}\right) \mathrm{Cu}\left(\mathrm{O}_{2}\right)$ (2) under $\mathrm{N}_{2}$, showing equilibration with $\mathrm{N}_{2}$ to yield ( $\left.{ }^{\mathrm{EMind}} \mathrm{L}\right) \mathrm{Cu}\left(\mathrm{O}_{2}\right)(\mathbf{1})$ in $c a .10 \%$ yield. 

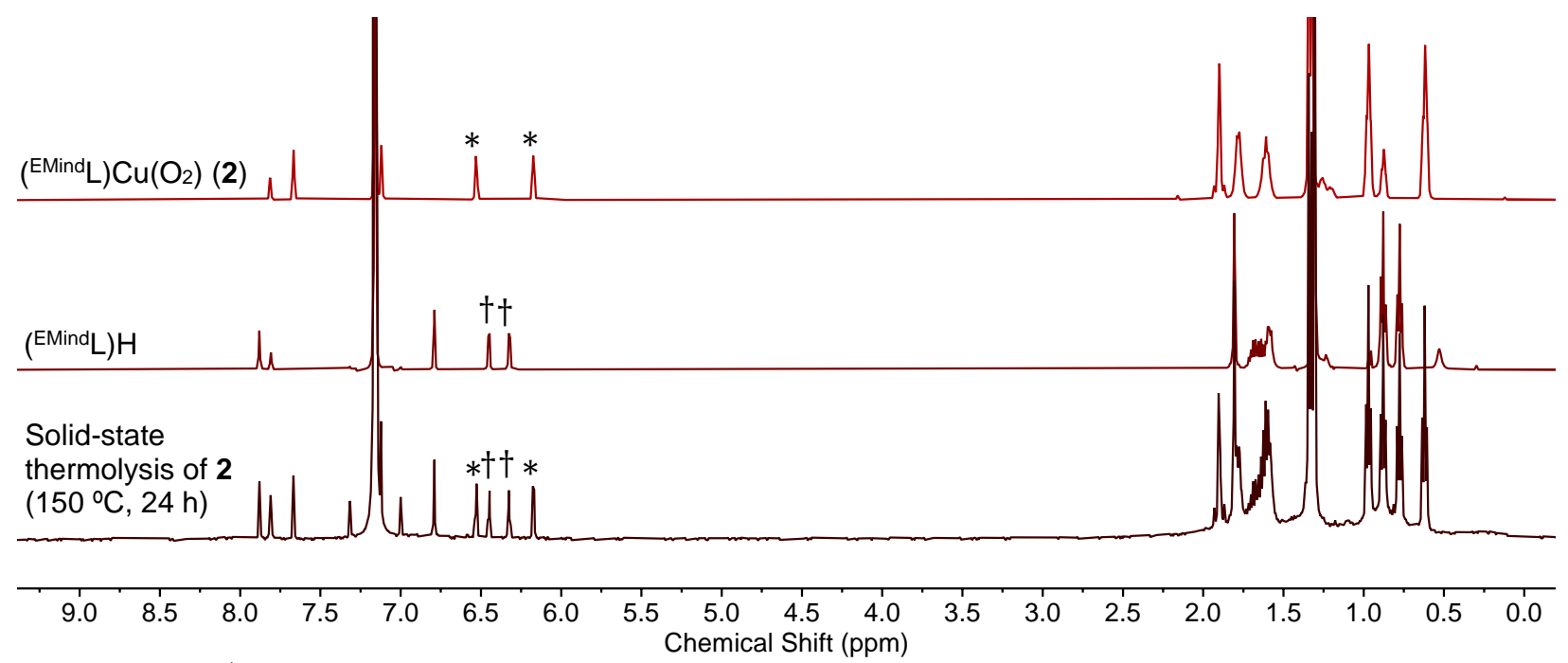

Figure S42. ${ }^{1} \mathrm{H}$ NMR spectra $\left(500 \mathrm{MHz}, \mathrm{C}_{6} \mathrm{D}_{6}\right)$ post solid-state thermolysis of $2\left(150{ }^{\circ} \mathrm{C}, 24 \mathrm{~h}\right)$, illustrating partial formation of $\left({ }^{\text {EMind }} \mathrm{L}\right) \mathrm{H}(\mathrm{ca} .47 \%$ yield $)$ with partially unconverted ( $\left.{ }^{\mathrm{EMind}} \mathrm{L}\right) \mathrm{Cu}\left(\mathrm{O}_{2}\right)(2)$. Diagnostic resonances of ( $\left.{ }^{\mathrm{EMind}} \mathrm{L}\right) \mathrm{Cu}\left(\mathrm{O}_{2}\right)(2)$ are marked with an asterisk (*), and diagnostic resonances of $\left({ }^{\text {EMind }} \mathrm{L}\right) \mathrm{H}$ are marked with an obelisk $(\dagger)$.

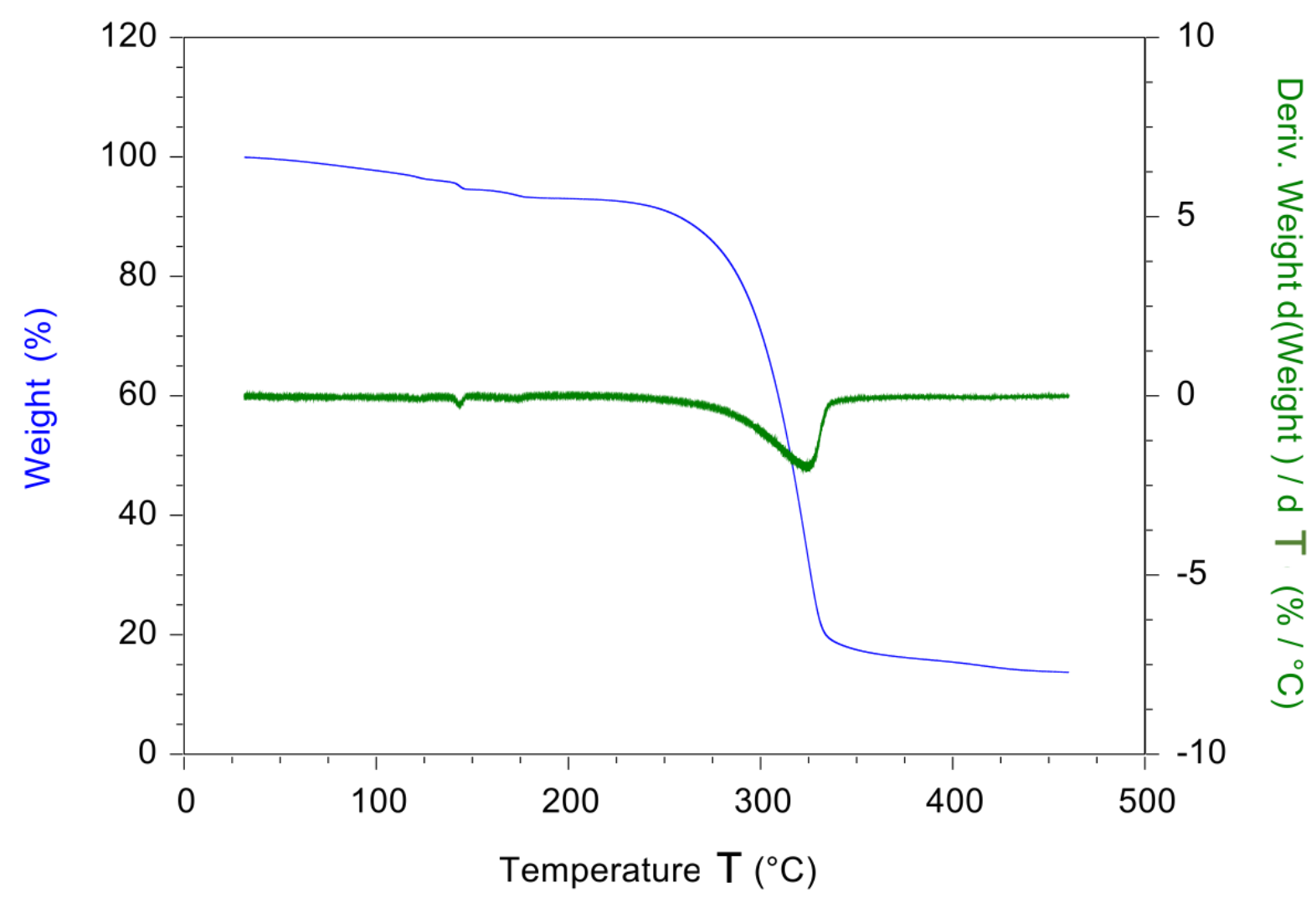

Figure S43. Thermogravimetric analysis (TGA) of crystalline $\left({ }^{\mathrm{EMind}} \mathrm{L}\right) \mathrm{Cu}\left(\mathrm{O}_{2}\right)(2)$ under Ar with increasing temperature. The initial drop in mass is due to loss of pentane from the unit cell, followed by an event at $140{ }^{\circ} \mathrm{C}$ associated with partial decomposition. Ligand decomposition is observed at $\mathrm{ca} .280^{\circ} \mathrm{C}$. The heating rate was set at $1{ }^{\circ} \mathrm{C} / \mathrm{min}$. 


\section{Single-Crystal-to-Single-Crystal Transformation.}

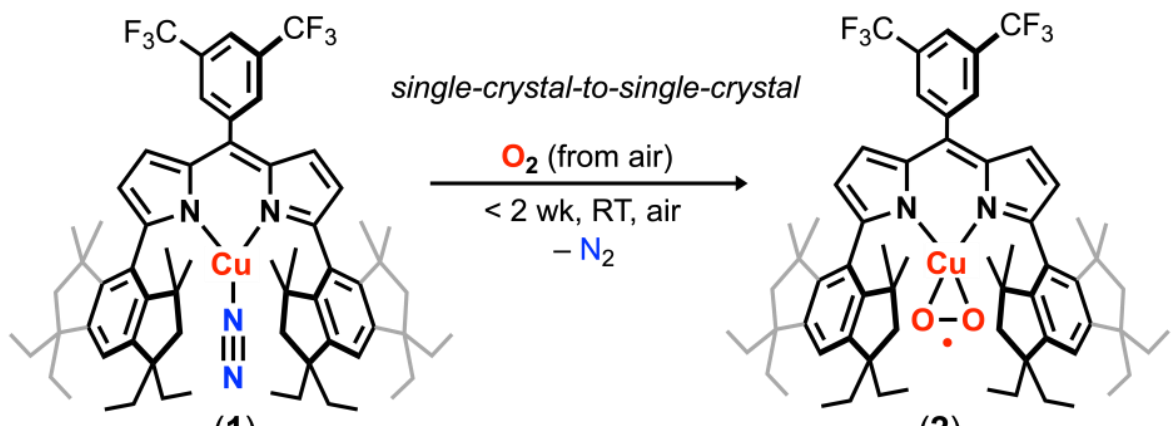

(1)

(2)

General Procedure. A dataset for a crystal of $\left({ }^{\mathrm{EMind}} \mathrm{L}\right) \mathrm{Cu}\left(\mathrm{N}_{2}\right)(\mathbf{1})$ was collected. The crystal, kept on the MiTeGen pin with Fluorolube oil, was allowed to stand under air for two weeks, and the data set was recollected and compared to an authentic dataset of $\left({ }^{\mathrm{EMind}} \mathrm{L}\right) \mathrm{Cu}\left(\mathrm{O}_{2}\right)(\mathbf{2})$.

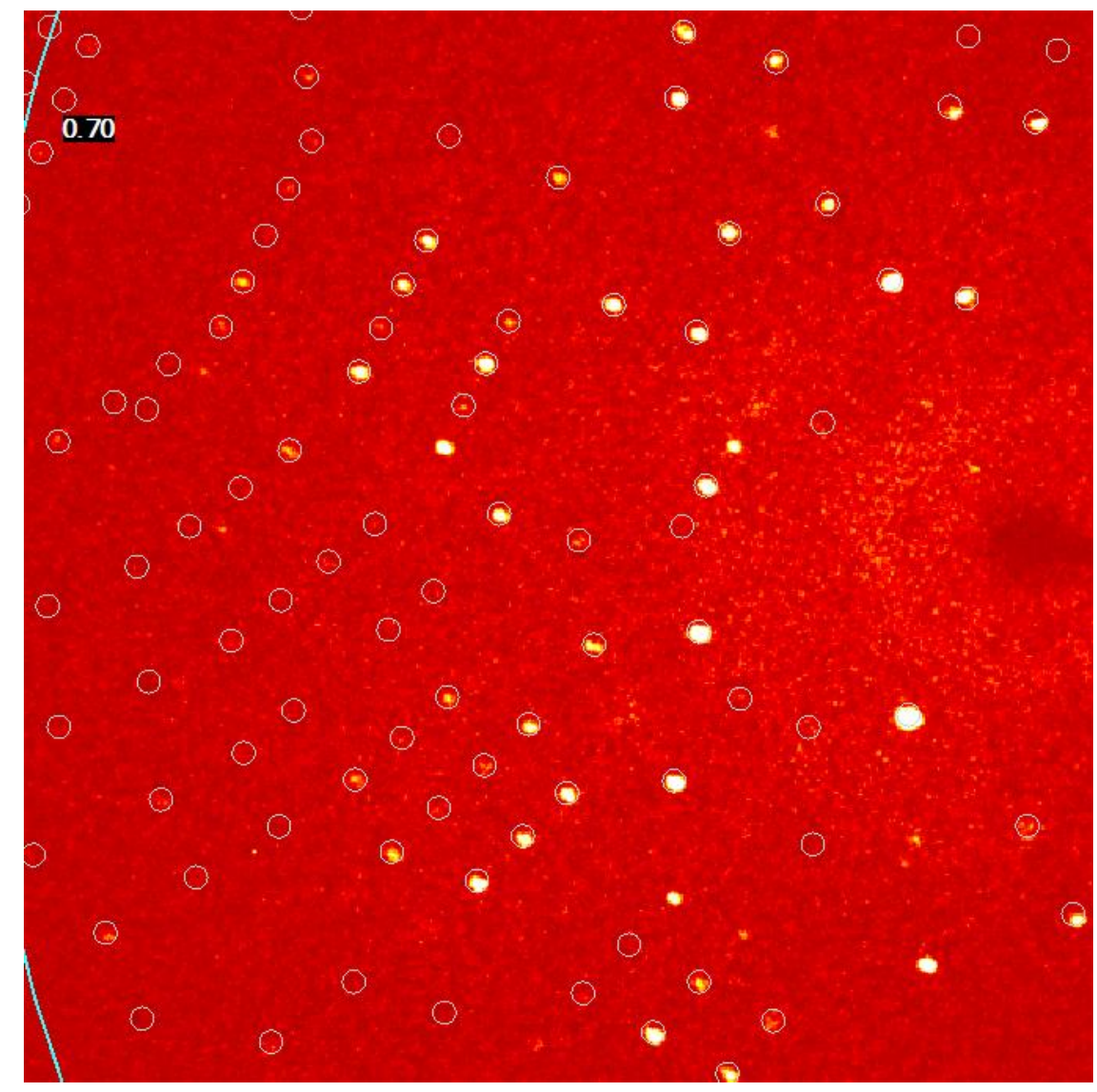

Figure S44. Single crystal of $\left({ }^{\mathrm{EMind}} \mathrm{L}\right) \mathrm{Cu}\left(\mathrm{N}_{2}\right)(\mathbf{1})$ allowed to stand on MiTeGen pin for two weeks under air, solved as $\left({ }^{\mathrm{EMind}} \mathrm{L}\right) \mathrm{Cu}\left(\mathrm{O}_{2}\right)(\mathbf{2})$ with no detectable electron density attributable for the $\mathrm{N} 2$ fragment. The above frame ( 2 second exposure, $0.70 \AA$ resolution) is displayed as a reference point for the single-crystal-to-single-crystal transformation. 


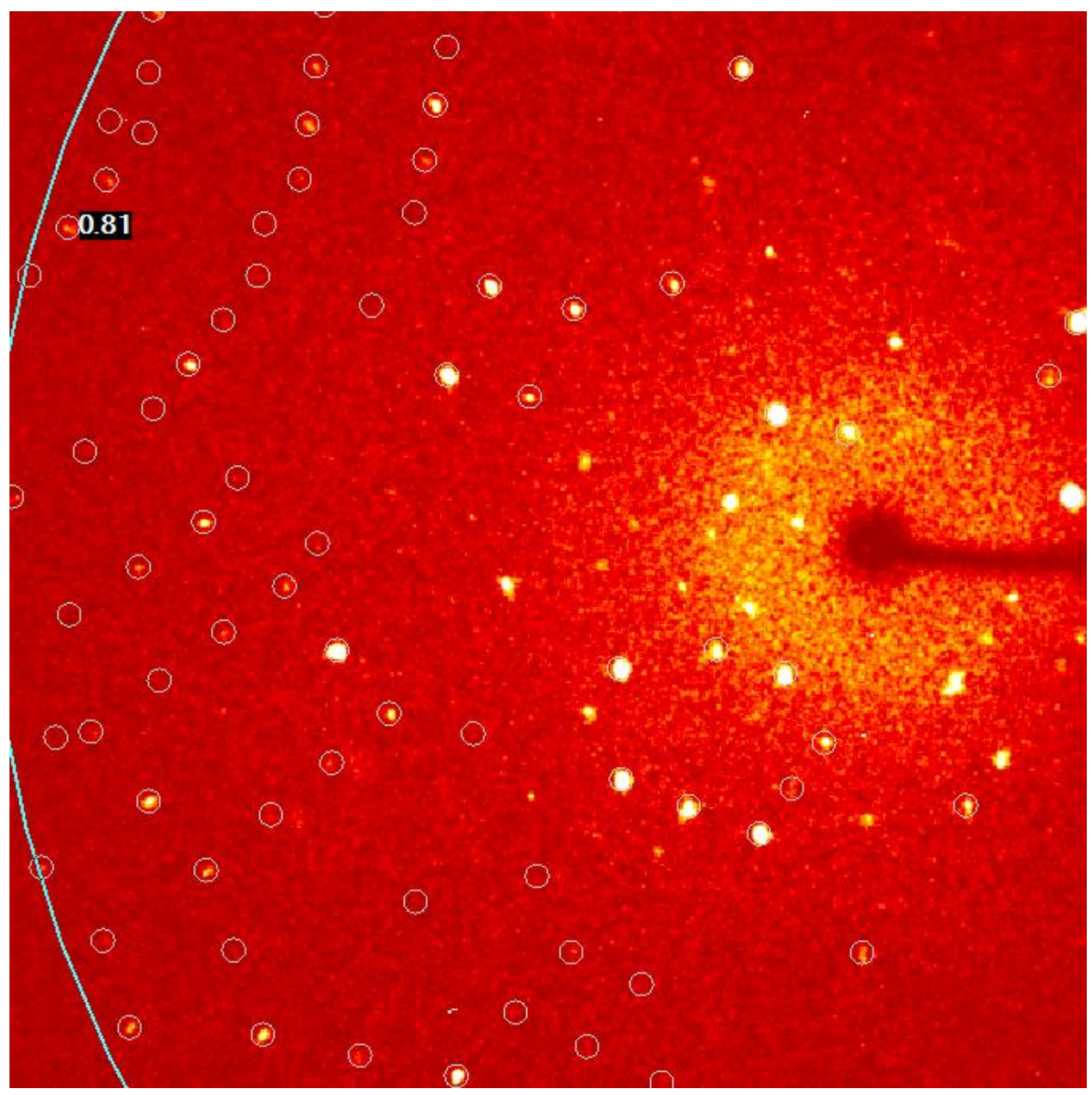

Figure S45. Single crystal of $\left({ }^{E M i n d} \mathrm{~L}\right) \mathrm{Cu}\left(\mathrm{N}_{2}\right)(\mathbf{1})$ allowed to stand on MiTeGen pin in a vacuum desiccator for 6 days. The above frame (10 second exposure, $0.81 \AA$ resolution $)$ displays crystallinity preserved under prolonged vacuum exposure. 


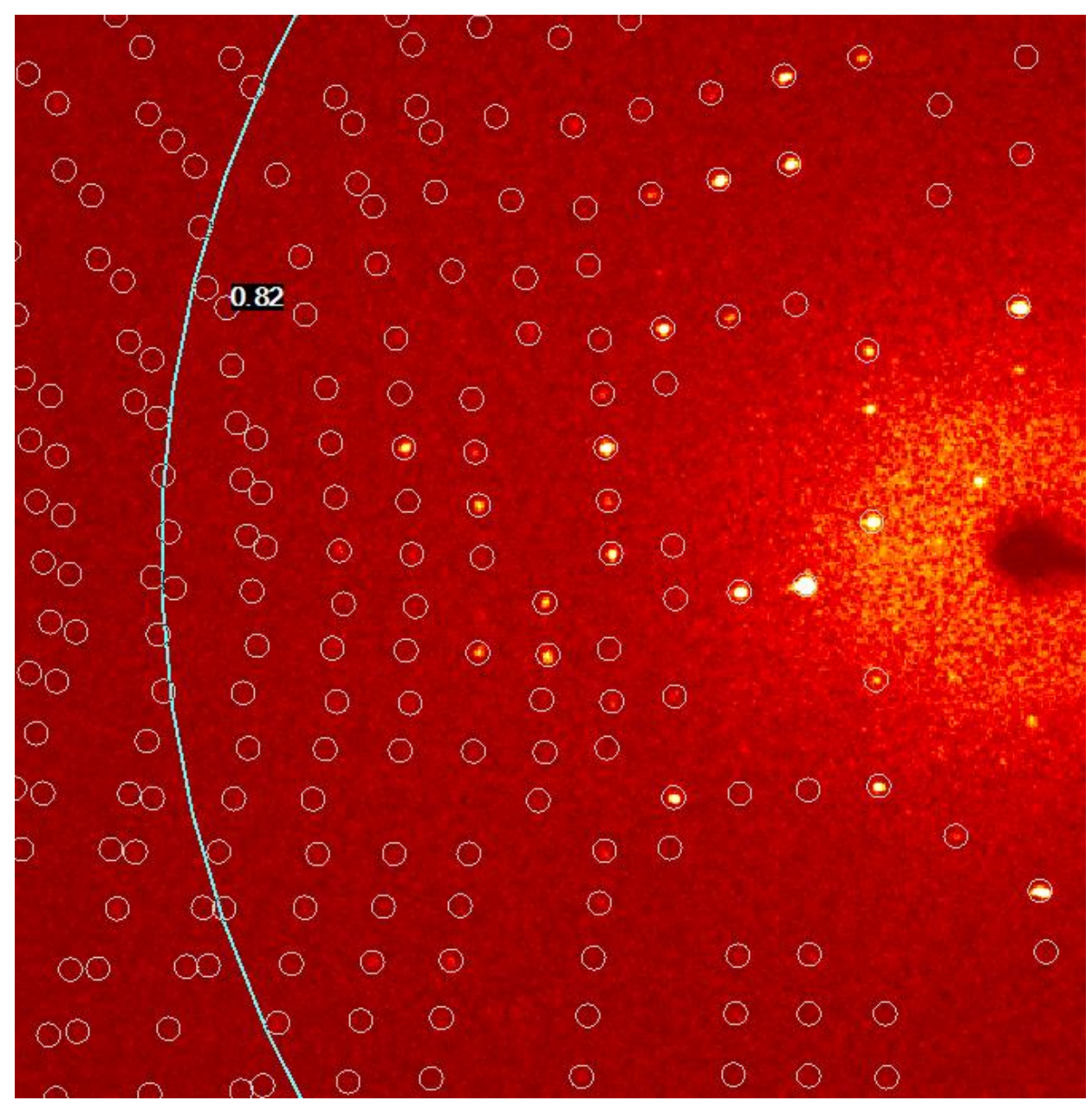

Figure S46. Single crystal of $\left({ }^{\mathrm{EMind}} \mathrm{L}\right) \mathrm{Cu}\left(\mathrm{N}_{2}\right)$ (1) allowed to stand on MiTeGen pin for two weeks under air, solved as $\left({ }^{\mathrm{EMind}} \mathrm{L}\right) \mathrm{Cu}\left(\mathrm{N}_{2}\right)(2)$ with no detectable electron density attributable for the $\mathrm{N} 2$ fragment. The above frame ( 1 second exposure, $0.82 \AA$ resolution) depicts minimal degradation and preserved crystallinity. 

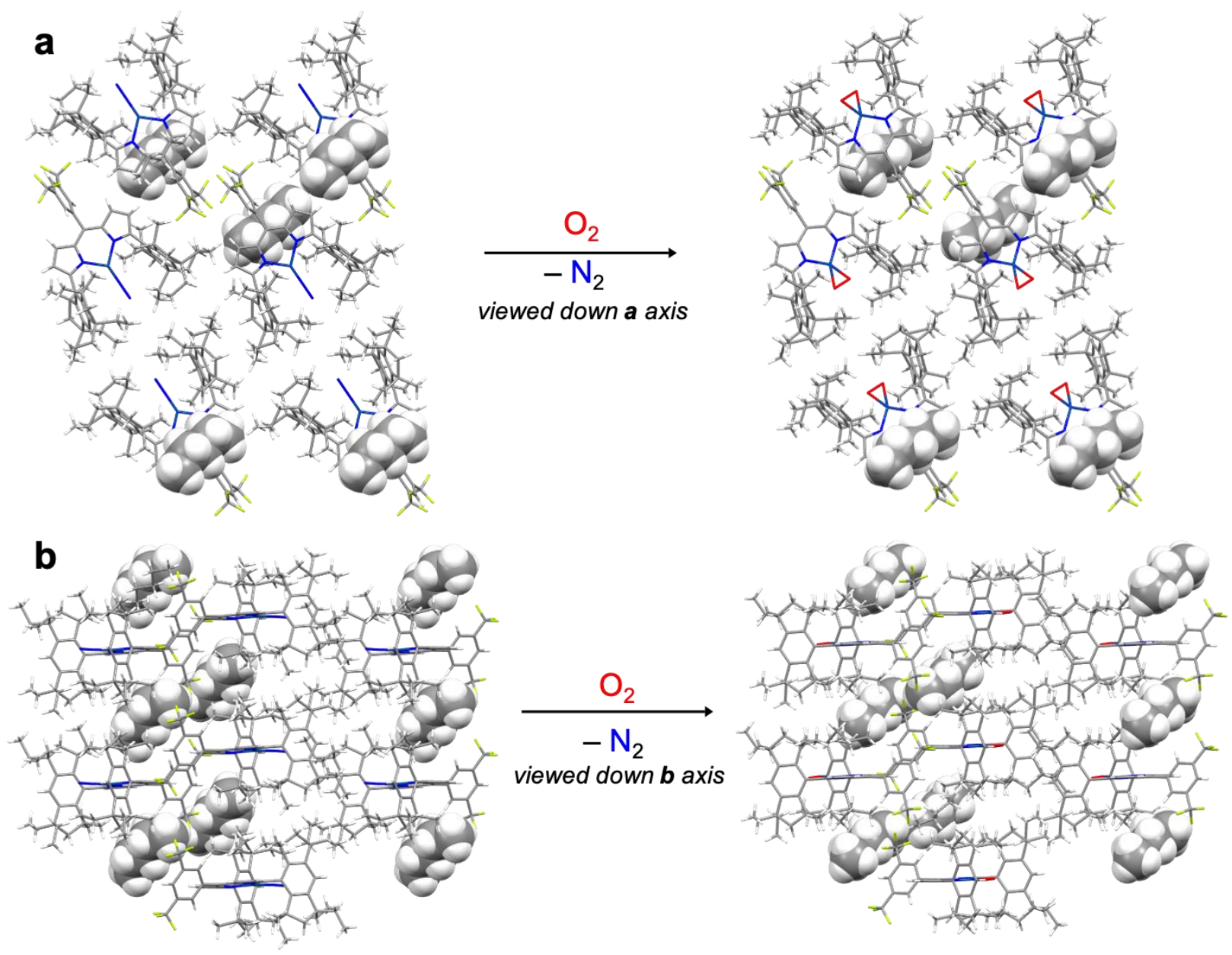

Figure S47. Depiction of pentane solvent within crystalline lattice of single-crystal-to-singlecrystal conversion of $\left({ }^{\mathrm{EMind}} \mathrm{L}\right) \mathrm{Cu}\left(\mathrm{N}_{2}\right)(\mathbf{1})$ to $\left({ }^{\mathrm{EMind}} \mathrm{L}\right) \mathrm{Cu}\left(\mathrm{N}_{2}\right)(\mathbf{2})$. The depiction down the a axis (top) and $\mathbf{b}$ axis (bottom) reveal the pentane solvent molecule is spatially confined between the dipyrrin $\pi$ system and the fluorinated aryl motif, resulting in low volatility under low pressure. 


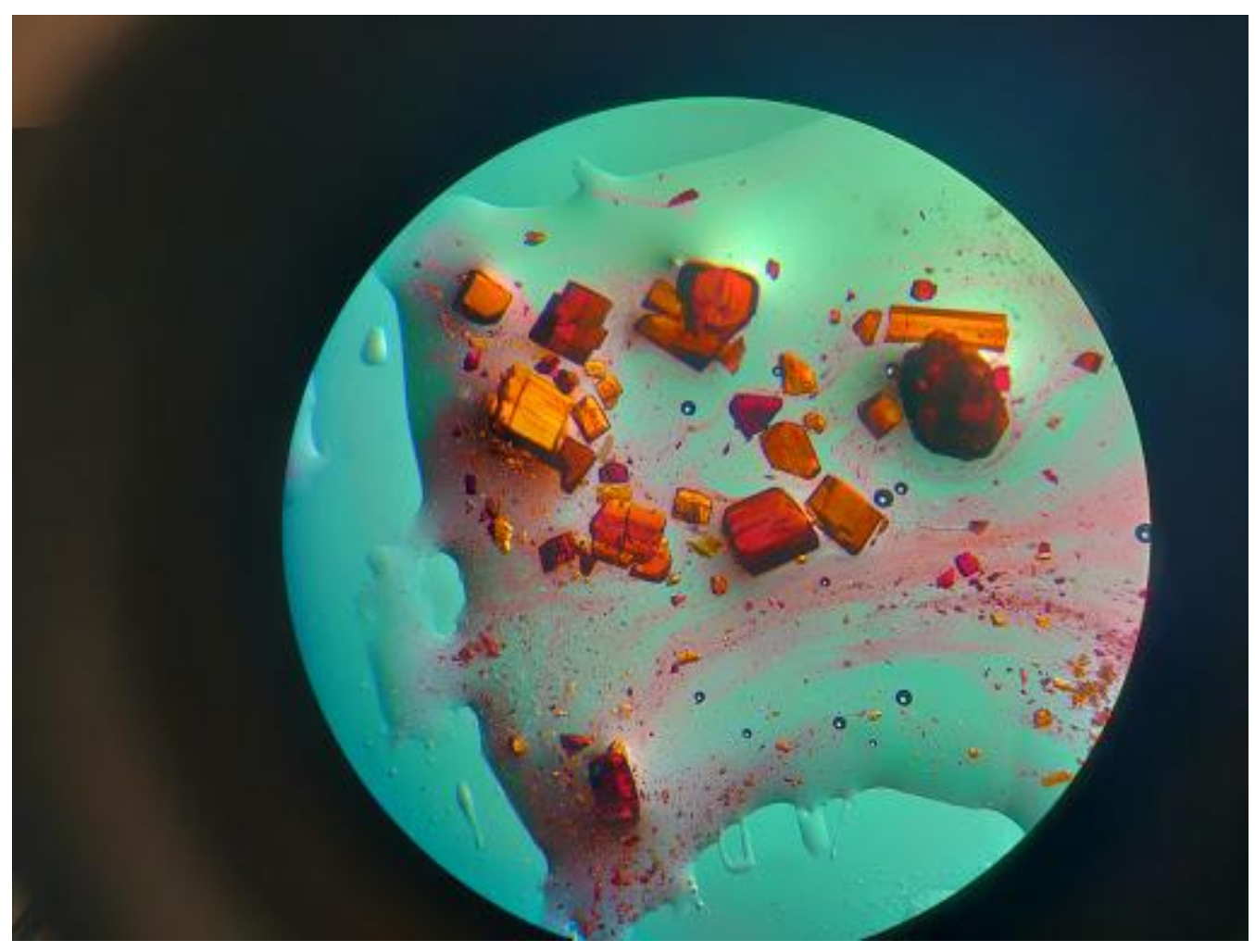

Figure S48. Snapshot of single-crystal-to-single crystal transformation ( $c a .4$ days), revealing maintained crystallinity and onset color change attributed to formation of $\left({ }^{\mathrm{EMind}} \mathrm{L}\right) \mathrm{Cu}\left(\mathrm{O}_{2}\right)(\mathbf{2})$ 


\section{1,2-Diphenylhydrazine Oxidation with ( $\left.{ }^{\mathrm{EMind}} \mathrm{L}\right) \mathrm{Cu}\left(\mathrm{O}_{2}\right)$ (2) Under Air}

$$
\text { PhNHNHPh } \underset{\mathrm{CH}_{2} \mathrm{Cl}_{2}, 2 \mathrm{~h}, \mathrm{RT} \text {, air }}{\left.\stackrel{[\text { EMind }}{\mathrm{C}} \mathrm{Cu}\left(\mathrm{O}_{2}\right)\right](1 \mathrm{~mol} \%)} \text { PhNNPh (94\%) }+\mathrm{H}_{2} \mathrm{O}_{2}
$$

General Procedure. In a dinitrogen-filled drybox, crystalline 1,2-diphenylhydrazine was measured into a $20 \mathrm{~mL}$ scintillation vial. A stir bar was added, and the vial was exported from the drybox. Meanwhile, $\left({ }^{\text {EMind }} \mathrm{L}\right) \mathrm{Cu}\left(\mathrm{O}_{2}\right)(2)$ was measured out in air and dissolved in the appropriate solvent (ca. $2 \mathrm{~mL}$ ). The scintillation vial containing the arylhydrazine was opened to air, and the stock solution of $\left({ }^{\text {EMind }} \mathrm{L}\right) \mathrm{Cu}\left(\mathrm{O}_{2}\right)$ was rapid added with stirring $(1000 \mathrm{rpm})$, resulting in a rapid color change to brown-orange attributed to azoarene formation. The reaction was allowed to continue stirring without a vial cap. Aliquots were removed at the appropriate time point with removal of solvent, and the sample was dissolved in $\mathrm{C}_{6} \mathrm{D}_{6}$ and checked by multinuclear NMR spectroscopy. Control experiments, conducted in the absence of $\mathbf{2}$ with otherwise identical rapid stirring and reagent quantities, establish the paramount role of $\mathbf{2}$ in facilitating the reaction (see Fig. S49 and Note $I$ ).

Note I - The rapidity of 1,2-diphenylhydrazine oxidation was observed to be highly sensitive to speed of stirring and shape of reaction vessel, attributed to rate of solution oxygenation. In accord, reactions set up in NMR tubes were prohibitively slow with constant inversion of the tube (attributed to poor $\mathrm{O}_{2}$ diffusion into solution based on minimal exposure of surface area of the solution to air), hindering a detailed kinetic analysis.

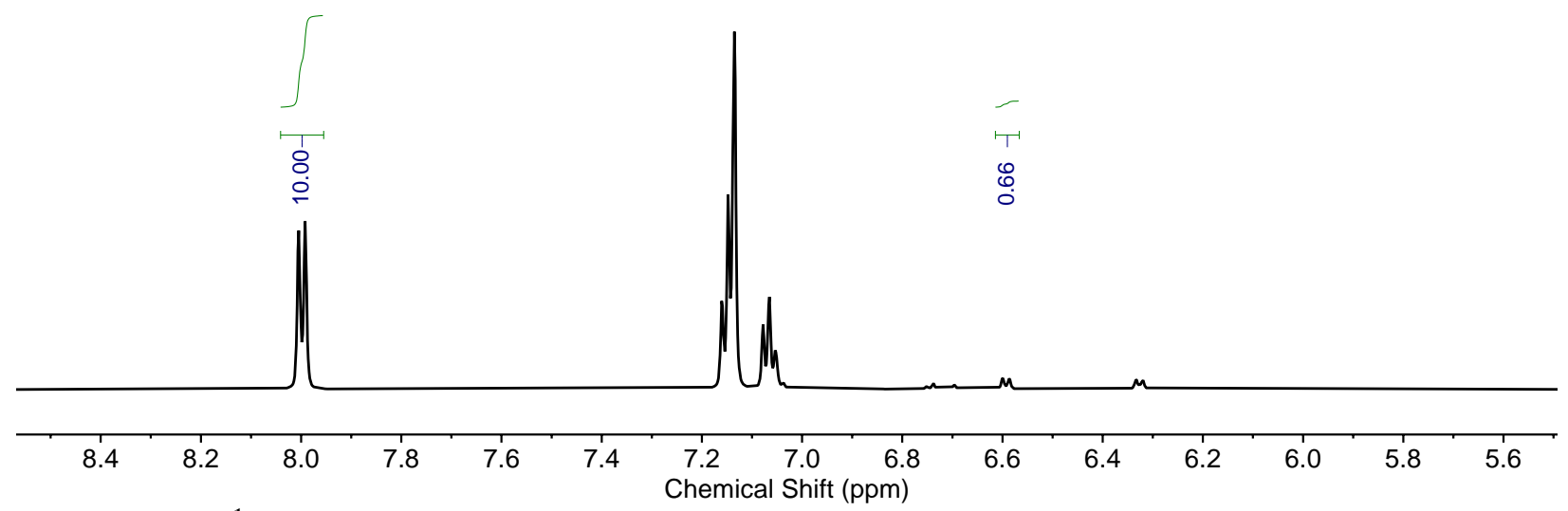

Figure S49. ${ }^{1} \mathrm{H}$ NMR spectrum $\left(600 \mathrm{MHz}, \mathrm{C}_{6} \mathrm{D}_{6}\right)$ of 1,2-diphenylhydrazine oxidation due to formation of $\left({ }^{\mathrm{EMind}} \mathrm{L}\right) \mathrm{Cu}\left(\mathrm{O}_{2}\right)(2)$ in dichloromethane after $2 \mathrm{~h}$. Baseline resonances correspond to dipyrrin species. 


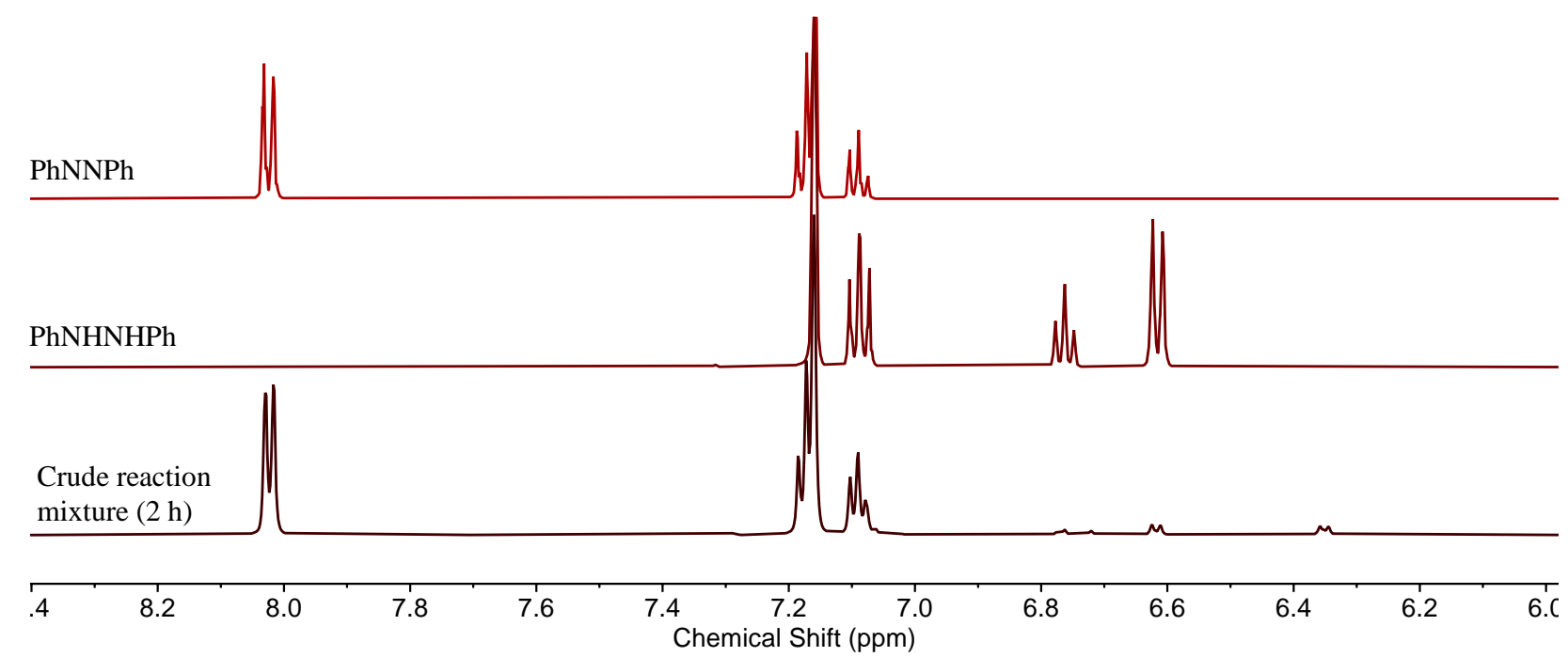

Figure S50. ${ }^{1} \mathrm{H}$ NMR spectrum $\left(600 \mathrm{MHz}, \mathrm{C}_{6} \mathrm{D}_{6}\right)$ of 1,2-diphenylhydrazine oxidation due to formation of $\left({ }^{\text {EMind }} \mathrm{L}\right) \mathrm{Cu}\left(\mathrm{O}_{2}\right)(2)$ in dichloromethane after $2 \mathrm{~h}$.

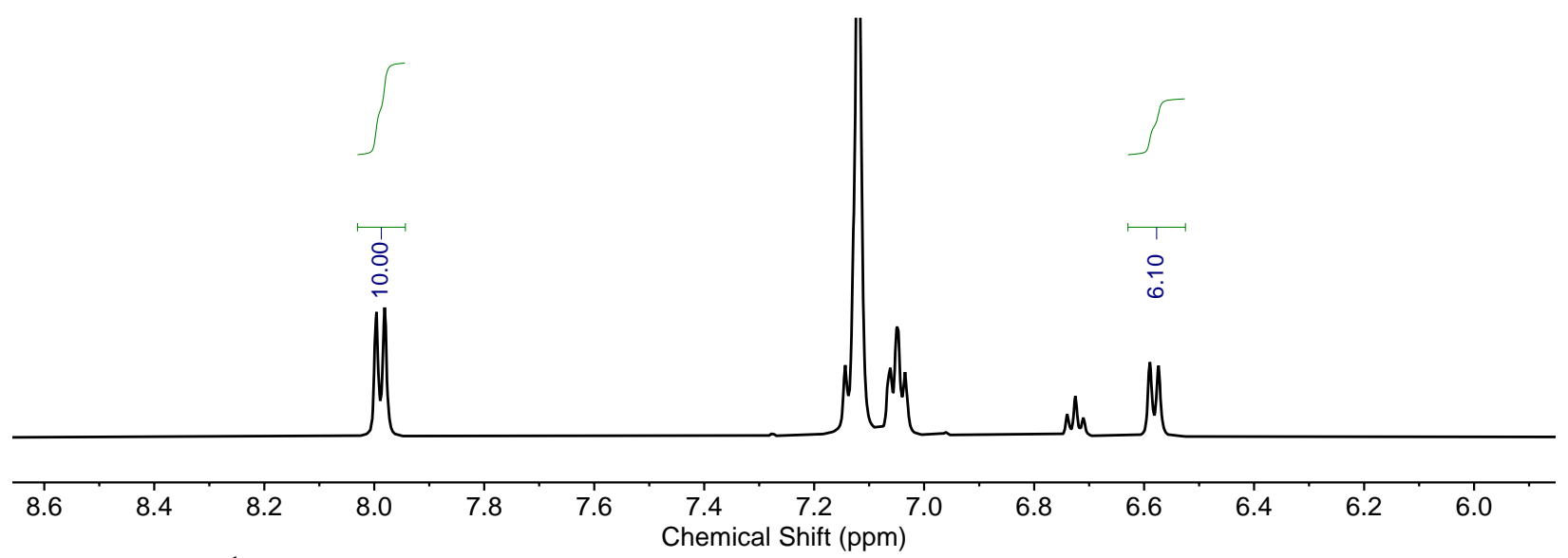

Figure S51. ${ }^{1} \mathrm{H}$ NMR spectrum $\left(500 \mathrm{MHz}, \mathrm{C}_{6} \mathrm{D}_{6}\right)$ of 1,2-diphenylhydrazine oxidation due to formation of $\left({ }^{\mathrm{EMind}} \mathrm{L}\right) \mathrm{Cu}\left(\mathrm{O}_{2}\right)(2)$ in hexanes after $2 \mathrm{~h}$. 


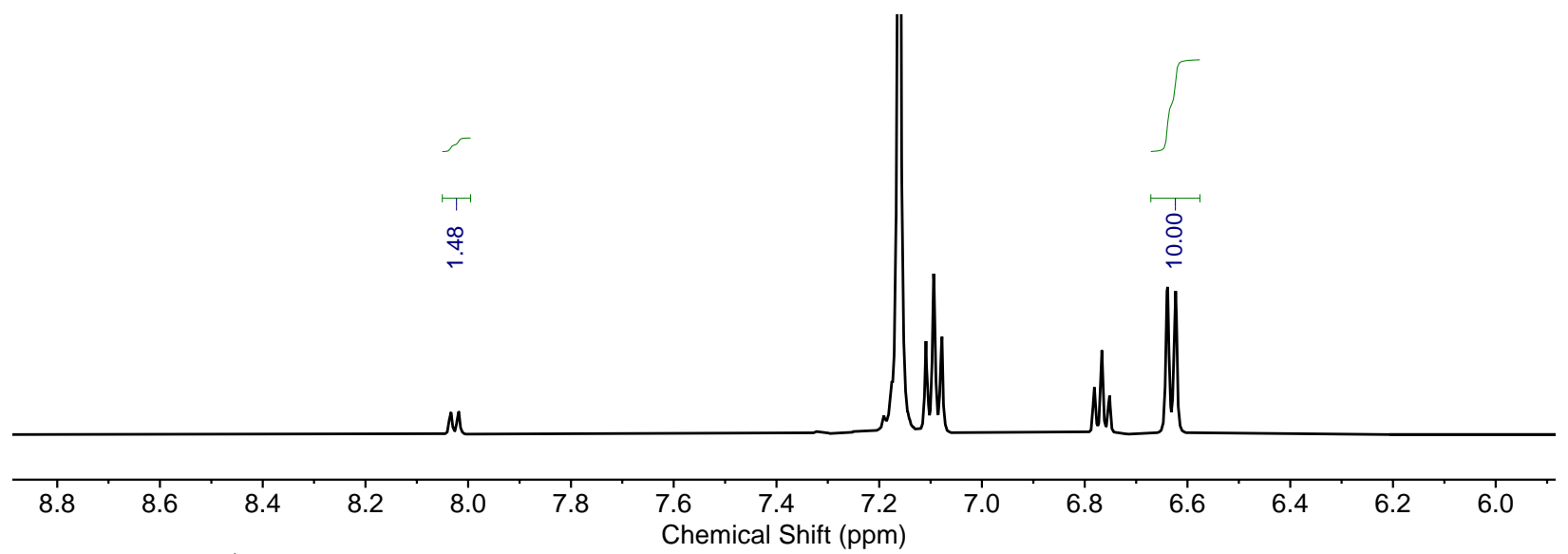

Figure S52. ${ }^{1} \mathrm{H}$ NMR spectrum $\left(500 \mathrm{MHz}, \mathrm{C}_{6} \mathrm{D}_{6}\right)$ of 1,2-diphenylhydrazine auto-oxidation in dichloromethane in the absence of $\left({ }^{\mathrm{EMind}} \mathrm{L}\right) \mathrm{Cu}\left(\mathrm{O}_{2}\right)(2)$ after $2 \mathrm{~h}$, revealing minor conversion to azoarene (ca. $13 \%$ yield).

Aqueous $\mathrm{H}_{2} \mathrm{O}_{2}$ added to $\mathrm{C}_{6} \mathrm{D}_{6}$

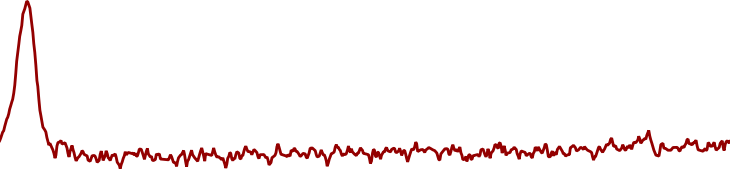

PhNHNHPh oxidation by 2 under air

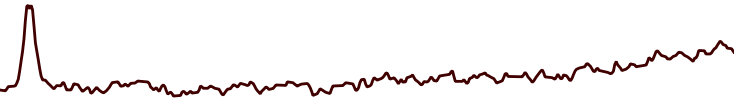

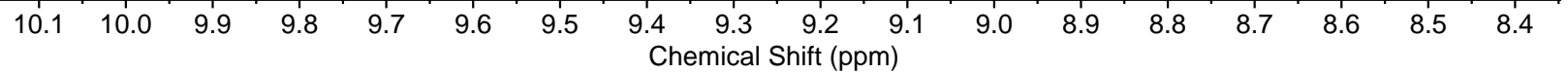

Figure S53. Truncated ${ }^{1} \mathrm{H}$ NMR spectra overlay $\left(500 \mathrm{MHz}, \mathrm{C}_{6} \mathrm{D}_{6}\right.$ ) of $\mathrm{H}_{2} \mathrm{O}_{2}$ in $\mathrm{C}_{6} \mathrm{D}_{6}$ (top, red) juxtaposed to crude 1,2-diphenylhydrazine oxidation (bottom, maroon), demonstrating $\mathrm{H}_{2} \mathrm{O}_{2}$ production. Due to the poor solubility of $\mathrm{H}_{2} \mathrm{O}_{2}$ in $\mathrm{C}_{6} \mathrm{D}_{6}$ and loss of $\mathrm{H}_{2} \mathrm{O}_{2}$ during workup step involving evacuation, quantification of $\mathrm{H}_{2} \mathrm{O}_{2}$ by ${ }^{1} \mathrm{H}$ NMR spectroscopy was not conducted. 


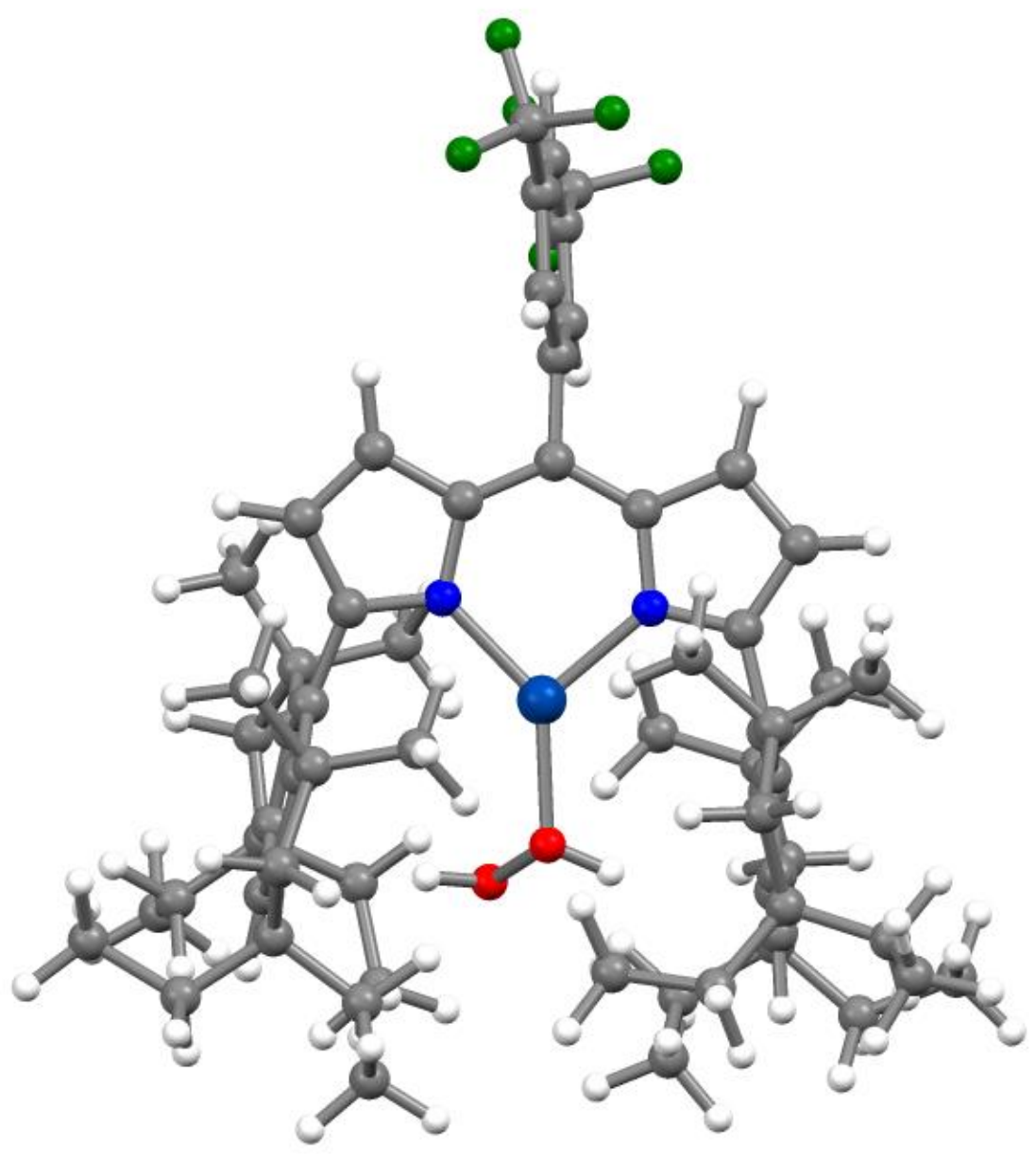

Figure S54. Geometry optimized structure of $\left({ }^{\mathrm{EMind}} \mathrm{L}\right) \mathrm{Cu}\left(\mathrm{H}_{2} \mathrm{O}_{2}\right)$ (7) with basis set/functional pairing lacv3p**/B3LYP-d3. ${ }^{6-10}$ Computations were conducted with the software Jaguar. ${ }^{11}$ Successful ground-state optimizations were assessed by the absence of negative frequencies and by the forces on the all atoms residing below $10^{-5}$ hartrees/bohr as well as total energy remaining unchanged by $10^{-4}$ between the final SCF iterations. Color Scheme: $\mathrm{Cu}$ (cobalt blue), F (green) $\mathrm{N}$ (blue), $\mathrm{O}$ (red). Geometry of ( $\left.{ }^{\mathrm{EMind}} \mathrm{L}\right) \mathrm{Cu}\left(\mathrm{O}_{2}\right)(\mathbf{2})$ was employed for initial coordinates.

Pertinent bond metrics include the following, which are in agreement with a cuprous ion in the dipyrrin framework with an unactivated hydrogen peroxide motif:

$\mathrm{Cu}-\mathrm{N}_{\text {dipyrrin: }}: 1.997 \AA, 1.998 \AA$

$\mathrm{Cu}-\mathrm{O}: 2.034 \AA$

O-O: $1.471 \AA$ (crystalline hydrogen peroxide, do-o: $1.453(7) \AA)^{12}$

$\Sigma(\angle \mathrm{Cu}): 360^{\circ}$ 
Cartesian Coordinates (x,y,z) - Optimization of $\left({ }^{\mathrm{EMind}} \mathrm{L}\right) \mathrm{Cu}\left(\mathrm{H}_{2} \mathrm{O}_{2}\right)(\mathbf{7})$

\begin{tabular}{|c|c|c|c|}
\hline $\mathrm{Cu} 1$ & 4.410110 & 7.361436 & 16.594389 \\
\hline $\mathrm{F} 2$ & 7.405112 & 1.153821 & 10.874097 \\
\hline $\mathrm{F} 3$ & 1.426011 & 2.234660 & 10.999123 \\
\hline F4 & 8.011450 & 3.140978 & 10.233004 \\
\hline $\mathrm{F} 5$ & 8.216287 & 2.573080 & 12.321695 \\
\hline N6 & 4.776037 & 7.669791 & 14.655753 \\
\hline F7 & 2.125394 & 3.375386 & 9.286696 \\
\hline F8 & 2.779040 & 1.315507 & 9.553522 \\
\hline C9 & 3.793740 & 4.918508 & 18.698532 \\
\hline C10 & 6.267951 & 3.919119 & 18.761608 \\
\hline C11 & 7.418580 & 2.456356 & 11.237449 \\
\hline $\mathrm{C} 12$ & 2.829969 & 5.670000 & 19.406300 \\
\hline C13 & 7.850808 & 13.739378 & 16.264208 \\
\hline H14 & 8.468954 & 13.275910 & 15.487411 \\
\hline H15 & 8.205877 & 14.767121 & 16.396055 \\
\hline H16 & 6.826072 & 13.781666 & 15.884068 \\
\hline $\mathrm{C} 17$ & 1.403875 & 6.057064 & 18.989836 \\
\hline C18 & 5.139232 & 10.014306 & 14.975759 \\
\hline C19 & 4.527679 & 4.410795 & 12.751843 \\
\hline $\mathrm{C} 20$ & 5.967577 & 2.491439 & 18.267862 \\
\hline $\mathrm{H} 21$ & 6.910164 & 1.943927 & 18.146947 \\
\hline $\mathrm{H} 22$ & 5.452766 & 2.488530 & 17.307230 \\
\hline $\mathrm{H} 23$ & 5.351419 & 1.945449 & 18.990971 \\
\hline $\mathrm{C} 24$ & 3.443510 & 3.453537 & 15.309607 \\
\hline $\mathrm{H} 25$ & 3.295710 & 2.709504 & 14.540023 \\
\hline $\mathrm{C} 26$ & 6.782768 & 4.968684 & 21.000224 \\
\hline $\mathrm{C} 27$ & 5.118961 & 7.307800 & 12.424210 \\
\hline $\mathrm{H} 28$ & 5.187602 & 6.804889 & 11.469266 \\
\hline C29 & 7.173275 & 3.823011 & 20.028088 \\
\hline H30 & 6.977403 & 2.862732 & 20.521065 \\
\hline H31 & 8.236069 & 3.833924 & 19.764154 \\
\hline C32 & 4.355509 & 5.364636 & 13.892347 \\
\hline C33 & 1.649169 & 11.463897 & 15.385341 \\
\hline H34 & 0.946836 & 12.043374 & 14.77839 \\
\hline H35 & 1.041584 & 10.793836 & 16.001761 \\
\hline C36 & 7.692889 & 6.211170 & 20.743917 \\
\hline H37 & 8.737131 & 5.888294 & 20.840839 \\
\hline H38 & 7.566685 & 6.520204 & 19.702264 \\
\hline C39 & 3.876884 & 11.685090 & 16.229773 \\
\hline
\end{tabular}




\begin{tabular}{|c|c|c|c|}
\hline $\mathrm{C} 40$ & 3.911075 & 4.793123 & 15.103036 \\
\hline N41 & 3.968531 & 5.428945 & 16.343191 \\
\hline $\mathrm{C} 42$ & 8.487527 & 10.630238 & 16.671862 \\
\hline $\mathrm{H} 43$ & 9.188069 & 10.069385 & 17.297839 \\
\hline H44 & 9.095897 & 11.290484 & 16.045652 \\
\hline $\mathrm{C} 45$ & 3.675489 & 2.951911 & 11.017975 \\
\hline $\mathrm{C} 46$ & 4.755666 & 6.693975 & 13.668468 \\
\hline $\mathrm{C} 47$ & 7.922921 & 12.970792 & 17.589097 \\
\hline H48 & 7.295503 & 13.482095 & 18.332520 \\
\hline H49 & 8.947883 & 13.017107 & 17.977220 \\
\hline C50 & 3.455719 & 3.914262 & 12.009506 \\
\hline H51 & 2.448177 & 4.259616 & 12.217121 \\
\hline C52 & 4.991507 & 11.971766 & 17.017419 \\
\hline H53 & 4.935225 & 12.721299 & 17.803212 \\
\hline C54 & 6.269741 & 10.315258 & 15.766838 \\
\hline C55 & 5.120034 & 8.825875 & 14.083615 \\
\hline C56 & 2.684073 & 13.806636 & 15.727323 \\
\hline H57 & 3.463729 & 14.316421 & 16.309243 \\
\hline H58 & 3.079049 & 13.724139 & 14.707949 \\
\hline C59 & 3.600899 & 4.538273 & 17.272607 \\
\hline C60 & 8.244290 & 4.120249 & 22.967137 \\
\hline H61 & 8.695951 & 3.352367 & 22.329355 \\
\hline H62 & 8.201280 & 3.721038 & 23.985993 \\
\hline H63 & 8.921544 & 4.980650 & 22.979485 \\
\hline C64 & 2.007493 & 7.008437 & 21.266700 \\
\hline C65 & 5.339592 & 8.650264 & 12.684454 \\
\hline H66 & 5.628778 & 9.420920 & 11.982608 \\
\hline C67 & 6.187462 & 11.293760 & 16.771322 \\
\hline C68 & 2.518960 & 12.368326 & 16.303535 \\
\hline O69 & 4.474103 & 8.713903 & 18.111953 \\
\hline $\mathrm{H} 70$ & 4.922963 & 9.555498 & 17.893191 \\
\hline C71 & 6.959205 & 4.704993 & 17.622081 \\
\hline H72 & 7.900689 & 4.214457 & 17.347623 \\
\hline H73 & 7.182756 & 5.734430 & 17.910688 \\
\hline H74 & 6.321259 & 4.751274 & 16.738127 \\
\hline C75 & 4.395743 & 5.996017 & 21.24982 \\
\hline H76 & 4.618701 & 6.421633 & 22.22359 \\
\hline C77 & 3.938423 & 10.715961 & 15.218629 \\
\hline C78 & 5.821463 & 3.940623 & 12.482819 \\
\hline H79 & 6.657484 & 4.309684 & 13.066186 \\
\hline
\end{tabular}




\begin{tabular}{|c|c|c|c|}
\hline $\mathrm{C} 80$ & 6.844102 & 4.504998 & 22.475525 \\
\hline H81 & 6.437418 & 5.287483 & 23.127869 \\
\hline H82 & 6.169012 & 3.646122 & 22.584044 \\
\hline C83 & 5.348112 & 5.242787 & 20.563657 \\
\hline O84 & 5.350943 & 8.202373 & 19.176951 \\
\hline H85 & 4.732139 & 7.585970 & 19.617055 \\
\hline C86 & 1.420972 & 14.674226 & 15.713105 \\
\hline H87 & 1.620501 & 15.628358 & 15.214037 \\
\hline H88 & 1.068896 & 14.900166 & 16.724293 \\
\hline H89 & 0.599258 & 14.189046 & 15.175138 \\
\hline C90 & 8.292586 & 9.622323 & 14.347407 \\
\hline H91 & 8.276262 & 10.612253 & 13.877676 \\
\hline H92 & 7.764717 & 8.925952 & 13.692293 \\
\hline H93 & 9.337407 & 9.297205 & 14.417304 \\
\hline C94 & 3.138913 & 6.189076 & 20.670646 \\
\hline C95 & 3.226318 & 3.303486 & 16.666638 \\
\hline H96 & 2.883392 & 2.420780 & 17.187537 \\
\hline C97 & 4.954650 & 2.467004 & 10.767814 \\
\hline H98 & 5.114060 & 1.698310 & 10.020927 \\
\hline C99 & 2.592687 & 10.602467 & 14.486690 \\
\hline C100 & 5.047737 & 4.684016 & 19.308145 \\
\hline C101 & 7.337366 & 10.913258 & 18.964916 \\
\hline H102 & 7.094834 & 9.847783 & 18.899727 \\
\hline H103 & 6.464683 & 11.395329 & 19.427911 \\
\hline C104 & 7.498526 & 11.479452 & 17.523626 \\
\hline C105 & 6.027049 & 2.966353 & 11.509374 \\
\hline C106 & 1.392862 & 7.076292 & 17.827208 \\
\hline H107 & 2.017734 & 7.946055 & 18.052243 \\
\hline H108 & 0.370569 & 7.430084 & 17.647175 \\
\hline H109 & 1.762899 & 6.626276 & 16.903204 \\
\hline C110 & 7.633135 & 8.240783 & 16.342767 \\
\hline H111 & 8.654060 & 7.851161 & 16.435449 \\
\hline H112 & 7.065294 & 7.561159 & 15.703588 \\
\hline H113 & 7.175749 & 8.219785 & 17.336789 \\
\hline C114 & 1.955265 & 12.443708 & 17.745871 \\
\hline H115 & 2.512935 & 13.209163 & 18.303444 \\
\hline H116 & 0.922044 & 12.807923 & 17.688850 \\
\hline C117 & 7.462249 & 7.434116 & 21.636014 \\
\hline H118 & 6.453220 & 7.838179 & 21.515308 \\
\hline H119 & 8.160683 & 8.232325 & 21.363069 \\
\hline
\end{tabular}




\begin{tabular}{|c|c|c|c|}
\hline H120 & 7.616022 & 7.210237 & 22.696951 \\
\hline C121 & 0.563199 & 4.829714 & 18.591038 \\
\hline H122 & 0.939792 & 4.368840 & 17.674536 \\
\hline H123 & -0.478245 & 5.126497 & 18.419661 \\
\hline H124 & 0.575084 & 4.073233 & 19.383626 \\
\hline $\mathrm{C} 125$ & 1.978396 & 11.133705 & 18.541353 \\
\hline H126 & 2.998280 & 10.781641 & 18.716265 \\
\hline H127 & 1.444559 & 10.326988 & 18.028073 \\
\hline H128 & 1.499989 & 11.270123 & 19.515754 \\
\hline C129 & 2.502021 & 2.461858 & 10.211301 \\
\hline C130 & 7.663989 & 9.670699 & 15.751742 \\
\hline C131 & 2.713823 & 11.200417 & 13.066457 \\
\hline H132 & 1.737586 & 11.191576 & 12.566821 \\
\hline H133 & 3.410078 & 10.614117 & 12.459574 \\
\hline H134 & 3.072408 & 12.234152 & 13.092585 \\
\hline C135 & 0.829755 & 6.707516 & 20.293469 \\
\hline H136 & 0.256694 & 7.610103 & 20.061639 \\
\hline H137 & 0.128734 & 6.012700 & 20.765484 \\
\hline C138 & 8.548012 & 11.082858 & 19.889926 \\
\hline H139 & 9.454778 & 10.647600 & 19.454850 \\
\hline H140 & 8.367189 & 10.578397 & 20.844891 \\
\hline H141 & 8.756233 & 12.134907 & 20.108095 \\
\hline C142 & 2.025828 & 9.171686 & 14.380325 \\
\hline H143 & 2.087525 & 8.649262 & 15.340444 \\
\hline H144 & 2.554352 & 8.569754 & 13.640093 \\
\hline H145 & 0.971176 & 9.223308 & 14.082993 \\
\hline C146 & 2.431248 & 8.507458 & 21.226447 \\
\hline H147 & 3.395197 & 8.604195 & 21.745163 \\
\hline H148 & 2.621381 & 8.784253 & 20.182478 \\
\hline C149 & 1.683624 & 6.581951 & 22.722470 \\
\hline H150 & 0.751735 & 7.074815 & 23.025898 \\
\hline H151 & 2.464257 & 6.976845 & 23.387469 \\
\hline C152 & 1.554488 & 5.070254 & 22.949394 \\
\hline H153 & 0.797250 & 4.622172 & 22.296374 \\
\hline H154 & 1.262692 & 4.859783 & 23.983827 \\
\hline H155 & 2.499890 & 4.555081 & 22.753973 \\
\hline C156 & 1.437688 & 9.498823 & 21.839561 \\
\hline H157 & 0.457645 & 9.445904 & 21.352511 \\
\hline H158 & 1.805433 & 10.523745 & 21.726915 \\
\hline H159 & 1.289592 & 9.319771 & 22.908868 \\
\hline
\end{tabular}


Table S2. X-ray diffraction experimental details. ${ }^{a}$

\begin{tabular}{|c|c|c|c|c|c|}
\hline & $\begin{array}{c}\left({ }^{\mathrm{EMind}} \mathrm{L}\right) \mathrm{Cu}\left(\eta^{2}-\mathrm{C}_{6} \mathrm{H}_{6}\right) \\
(\text { 1-benzene })-2049910\end{array}$ & $\begin{array}{c}\left({ }^{\mathrm{EMind}} \mathrm{L}\right) \mathrm{Cu}\left(\mathrm{O}_{2}\right) \\
(2)-2049906\end{array}$ & $\begin{array}{l}\left({ }^{\text {EMind }} \mathrm{L}\right) \mathrm{Cu}\left(\mathrm{CH}_{2} \mathrm{Cl}_{2}\right) \\
(\mathbf{4})-2049909\end{array}$ & $\begin{array}{c}\left({ }^{\mathrm{EMind}} \mathrm{L}\right) \mathrm{Cu}\left(\mathrm{NH}_{2} \mathrm{NH}_{2}\right) \\
(\mathbf{5})-2049908\end{array}$ & $\begin{array}{c}\left({ }^{\text {EMind }} \mathrm{L}\right) \mathrm{CuI} \\
(\mathbf{6})-2049907\end{array}$ \\
\hline $\begin{array}{c}\text { Moiety } \\
\text { Formula }\end{array}$ & $\mathrm{C}_{71} \mathrm{H}_{87} \mathrm{CuF}_{6} \mathrm{~N}_{2} \cdot \mathrm{C}_{6} \mathrm{H}_{6}$ & $\mathrm{C}_{65} \mathrm{H}_{81} \mathrm{CuF}_{6} \mathrm{~N}_{2} \mathrm{O}_{2} \cdot \mathrm{C}_{5} \mathrm{H}_{12}$ & $\mathrm{C}_{66} \mathrm{H}_{81} \mathrm{Cl}_{2} \mathrm{Cu}_{2} \mathrm{~N}_{2} \cdot \mathrm{C}_{5} \mathrm{H}_{12}$ & $\mathrm{C}_{65} \mathrm{H}_{85} \mathrm{CuF}_{6} \mathrm{~N}_{4} \cdot \mathrm{C}_{5} \mathrm{H}_{12}$ & $\mathrm{C}_{65} \mathrm{H}_{81} \mathrm{CuF}_{6} \mathrm{IN}_{2}$ \\
\hline FW & 1296.29 & 1172.02 & 1222.93 & 1172.07 & 1194.77 \\
\hline$\lambda(\mathbf{n m})$ & 0.71073 & 0.71073 & 0.71073 & 0.71073 & 0.71073 \\
\hline$T(\mathbf{K})$ & $100(2)$ & $100(2)$ & $100(2)$ & $100(2)$ & $100(2)$ \\
\hline Crystal System & Triclinic & Triclinic & Triclinic & Triclinic & Triclinic \\
\hline Space Group $(Z)$ & $\mathrm{P} \overline{1}(2)$ & $\mathrm{P} \overline{1}(2)$ & $\mathrm{P} \overline{1}(2)$ & $\mathrm{P} \overline{1}(2)$ & $\mathrm{P} \overline{1}(2)$ \\
\hline$a(\AA)$ & $14.0750(8)$ & $10.415(2)$ & $10.757(12)$ & $10.514(4)$ & $10.546(4)$ \\
\hline $\boldsymbol{b}(\AA)$ & $14.2433(8)$ & $13.866(3)$ & $14.73(2)$ & $13.806(3)$ & $13.838(6)$ \\
\hline$c(\AA)$ & $17.2305(10)$ & $22.041(5)$ & $22.63(2)$ & $22.372(5)$ & $22.418(8)$ \\
\hline$\alpha\left(^{\circ}\right)$ & $93.596(1)$ & $94.705(4)$ & $93.90(4)$ & $82.714(14)$ & $97.302(8)$ \\
\hline$\beta\left({ }^{\circ}\right)$ & $103.655(1)$ & $93.341(5)$ & $99.65(3)$ & $89.690(15)$ & $90.014(8)$ \\
\hline$\gamma\left({ }^{\circ}\right)$ & $99.936(1)$ & $97.663(4)$ & $96.60(5)$ & $82.004(11)$ & $97.506(8)$ \\
\hline Volume $\left(\AA^{3}\right)$ & $3286.9(3)$ & $3136.3(12)$ & $3498(7)$ & $3189.7(16)$ & $3217(2)$ \\
\hline Calc. $\rho\left(\mathrm{mg} / \mathrm{m}^{3}\right)$ & 1.237 & 1.241 & 1.161 & 1.220 & 1.233 \\
\hline$\mu\left(\mathbf{m m}^{-1}\right)$ & 0.394 & 0.412 & 0.444 & 0.403 & 0.874 \\
\hline Crystal Size (mm) & $0.16 \times 0.22 \times 0.23$ & $0.13 \times 0.22 \times 0.24$ & $0.17 \times 0.22 \times 0.23$ & $0.10 \times 0.10 \times 0.20$ & $0.12 \times 0.15 \times 0.40$ \\
\hline Reflections & 8322 & 7017 & 6913 & 7366 & 6338 \\
\hline 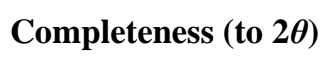 & 0.990 & 0.960 & 0.960 & 0.984 & 0.992 \\
\hline GOF on $F^{2}$ & 1.068 & 1.027 & 1.039 & 1.029 & 0.973 \\
\hline $\begin{array}{l}\mathbf{R} 1, w \mathbf{R}^{\mathbf{a}} \\
{[I>2 \sigma(I)]}\end{array}$ & $0.0469,0.1311$ & $0.0641,0.1685$ & $0.0879,0.2803$ & $0.0663,0.1391$ & $0.0778,0.1975$ \\
\hline
\end{tabular}

${ }^{\mathrm{a}} R 1=\sum|| F_{o}|-| F_{c}|| / \sum\left|F_{o}\right|, w R 2=\left\{\sum\left[w\left(F_{o}^{2}-F_{c}^{2}\right)^{2}\right] / \sum\left[w\left(F_{o}^{2}\right)^{2}\right]\right\}^{1 / 2}$ 
X-Ray Diffraction Techniques. All structures were collected on a Bruker three-circle platform goniometer equipped with an Apex II CCD and an Oxford cryostream-cooling device fixed at 100 K. Radiation was from a graphite fine focus sealed tube $\operatorname{Mo} \mathrm{K} \alpha(\lambda=0.71073 \AA)$ source (1-benzene, $\mathbf{2}, \mathbf{3}, \mathbf{4}, \mathbf{5}, \mathbf{6})$. The partial solubility of all complexes on the ( $\left.{ }^{\mathrm{EMind}} \mathrm{L}\right)$ platform in Paratone $-N$ oil forced us to employ an alternative crystal mounting procedure. Crystals were suspended in minimal thawing Fluorolube (stored in a vacuum desiccator or the drybox to minimize water content), quickly transferred to a dry ice bath outside the drybox ( $<10$ seconds transit time) and rapidly transferred to a room temperature microscope slide ( $c a .3$ minute transit time) for crystal selection with a glass fiber pin. At room temperature, crystals were observed to darken rapidly and lose the ability to polarize incidental light, attributed to desolvation of the unit cell and gradual dissolution into the surrounding oil. Such behavior was common for crystals isolated from slow cooling of concentrated hydrocarbon solutions, pentane in particular. Consequently, the crystal selection process was rapidly conducted at low temperatures, considering crystal size and crystal quality. For 1-benzene and 4, rapid degradation of the crystals was observed under ambient conditions due to the heightened sensitivity to $\mathrm{O}_{2}$ and $\mathrm{N}_{2}$. None of the crystals shows significant decay during the data collection.

Data was collected as a series of $\varphi$ and/or $\omega$ scans. Data integration down to $0.84 \AA$ resolution was carried out using SAINT V8.37 $\mathrm{A}^{13}$ with reflection spot size optimization. Absorption corrections were made with the program SADABS. ${ }^{14}$ Space group assignments were determined by examination of systematic absences, E-statistics, and successive refinement of the structures. The structure was solved by the Intrinsic Phasing methods and refined by least-squares methods against $F^{2}$ using SHELXT-2014 ${ }^{15}$ and SHELXL-2014 ${ }^{16}$ with the OLEX2 interface. ${ }^{17}$ The program PLATON was employed to confirm the absence of higher symmetry space groups. ${ }^{18}$ All non-H atoms, including the disorder fragments, were located in difference Fourier maps, and then refined anisotropically. The restraints on bond lengths and constraints of the atomic displacement parameters on each pair of disorder fragments (SADI/SAME and EADP instructions of SHELXL$2014)^{16}$ as well as the restraints of the atomic displacement parameters (SIMU/RIGU instructions of SHELXL-2014), if necessary, have been applied for the disorder refinement. ${ }^{19}$ All nonhydrogen atoms were refined anisotropically. Outlier reflections were omitted from refinement when appropriate. Hydrogen atoms on $\mathrm{C}$ atoms were placed at idealized positions and refined using a riding model. The isotropic displacement parameters of all hydrogen atoms were fixed to 1.2 times the atoms they are linked to (1.5 times for methyl groups). Crystallographic refinement details, including disorder modeling and software employed, have been delineated within in each crystallographic information file (*.cif).

Molecular graphics were generated using Povray V3.7. ${ }^{20}$ 
Further details on particular structures are noted below:

( $\left.{ }^{\text {EMind }} \mathbf{L}\right) \mathbf{C u}\left(\boldsymbol{\eta}^{2}-\mathbf{C}_{6} \mathbf{H}_{6}\right)$ (1-benzene). The structure was solved in the triclinic space group $\mathrm{P} \overline{1}$ with one molecule of copper-containing complex per asymmetric unit and two molecules per unit cell. One molecule of pentane was located and modelled appropriately. Positional disorder of the benzene ligand (C66-C71) over two positions was observed, which was modelled using similarity constraints and restraints. CCDC Identifier: 2049910.

( $\left.{ }^{\operatorname{EMind}} \mathbf{L}\right) \mathbf{C u}\left(\mathrm{O}_{2}\right)$ (2). The structure was solved in the triclinic space group P $\overline{1}$ with one molecule of copper-containing complex per asymmetric unit and two molecules per unit cell. One molecule of pentane was positionally disordered and modelled using similarity constraints and restraints. One of the trifluoromethyl substituents containing $\mathrm{C} 17$ was positionally and rotationally disordered and subsequently modelled using similarity constraints and restraints. Several reflects were coincidental with the beam-stop and omitted. CCDC Identifier: 2049906.

( $\left.{ }^{\text {EMind }} \mathbf{L}\right) \mathbf{C u}\left(\mathrm{CH}_{2} \mathbf{C l}_{2}\right)$ (4). The structure was solved in the triclinic space group $\mathrm{P} \overline{1}$ with one molecule of copper-containing complex per asymmetric unit and two molecules per unit cell. One molecule of pentane was located and modelled appropriately using the RIGU command. A second molecule of pentane was disordered about a special position and squeezed out using the Olex2 mask function to improve the $\mathrm{C}-\mathrm{C}$ bond precision. The ligated molecule of dichloromethane was disordered over two positions and modelled using similarly constraints and restraints. CCDC Identifier: 2049909

(EMind $\mathbf{L}) \mathbf{C u}\left(\mathrm{NH}_{2} \mathrm{NH}_{2}\right)(5)$. The structure was solved in the triclinic space group $\mathrm{P} \overline{1}$ with one molecule of copper-containing complex per asymmetric unit and two molecules per unit cell. Positional disorder of an ethyl motif containing C55 was modeled using similarity constraints and restraints. The trifluoromethyl motif containing $\mathrm{C} 16$ was positionally and rotationally disordered, modelled using similarity constraints and restraints. The hydrazine motif containing N3 was disordered over two positions and modelled appropriately. One molecule of pentane was located and modelled appropriately. CCDC Identifier: 2049908.

$\left({ }^{\text {EMind }} \mathbf{L}\right) \mathbf{C u I}(\mathbf{6})$. The structure was solved in the triclinic space group P $\overline{1}$ with one molecule of copper-containing complex per asymmetric unit and two molecules per unit cell. Positional disorder within the hydrindacene motif containing C58 was modeled using similarity constraints and restraints. One molecule of pentane was located and disordered about a special position (inversion center), which could not be satisfactorily modelled without diffuse ellipsoids. The solvent molecule was squeezed out using the Olex2 mask function to improve the $\mathrm{C}-\mathrm{C}$ bond precision. CCDC Identifier: 2049907. 
Table S3. Pertinent bond metrics of $\left({ }^{\mathrm{EMind}} \mathrm{L}\right) \mathrm{Cu}\left(\eta^{2}-\mathrm{C}_{6} \mathrm{H}_{6}\right)$ (1-benzene).

\begin{tabular}{lccl}
$\mathrm{Cu} 1-\mathrm{N} 1$ & $1.997(2) \AA$ & $\Sigma(\angle \mathrm{Cu} 1)$ & $360.5(8)^{\circ}$ \\
$\mathrm{Cu} 1-\mathrm{N} 2$ & $1.984(2) \AA$ & $\angle \mathrm{N} 1-\mathrm{Cu} 1-\mathrm{N} 2$ & $99.72(8)^{\circ}$ \\
$\mathrm{Cu} 1-\mathrm{C} 66$ & $2.19(1) \AA$ & $\mathrm{Cu} 1-\mathrm{C} 67$ & $2.24(2)^{\circ}$ \\
\hline
\end{tabular}

Table S4. Pertinent bond metrics of $\left({ }^{\mathrm{EMind}} \mathrm{L}\right) \mathrm{Cu}\left(\mathrm{O}_{2}\right)(\mathbf{2})$.

$\begin{array}{llcl}\mathrm{Cu} 1-\mathrm{N} 1 & 1.887(3) \AA & \Sigma(\angle \mathrm{Cu} 1) & 360.0(2)^{\circ} \\ \mathrm{Cu} 1-\mathrm{N} 2 & 1.885(3) \AA & \mathrm{O} 1-\mathrm{O} 2 & 1.378(4)^{\circ} \\ \mathrm{Cu} 1-\mathrm{O} 1 & 1.823(3) \AA & \mathrm{Cu} 1-\mathrm{O} 2 & 1.834(3)^{\circ}\end{array}$

Table S5. Pertinent bond metrics of $\left({ }^{\mathrm{EMind}} \mathrm{L}\right) \mathrm{Cu}\left(\mathrm{CH}_{2} \mathrm{Cl}_{2}\right)(4)$.

$\begin{array}{llcl}\mathrm{Cu} 1-\mathrm{N} 1 & 1.943(5) \AA & \Sigma(\angle \mathrm{Cu} 1) & 359.7(5)^{\circ} \\ \mathrm{Cu} 1-\mathrm{N} 2 & 1.949(5) \AA & \angle \mathrm{N} 1-\mathrm{Cu} 1-\mathrm{Cl} 1 & 118.4(3)^{\circ} \\ \mathrm{Cu} 1-\mathrm{Cl} 1 & 2.177(9) \AA & \angle \mathrm{N} 2-\mathrm{Cu} 1-\mathrm{Cl} 1 & 141.7(3)^{\circ}\end{array}$

Table S6. Pertinent bond metrics of $\left({ }^{\mathrm{EMind}} \mathrm{L}\right) \mathrm{Cu}\left(\mathrm{NH}_{2} \mathrm{NH}_{2}\right)($ 5).

$\begin{array}{lccc}\mathrm{Cu} 1-\mathrm{N} 1 & 1.955(3) \AA & \Sigma(\angle \mathrm{Cu} 1) & 359.8(4)^{\circ} \\ \mathrm{Cu} 1-\mathrm{N} 2 & 1.975(3) \AA & \angle \mathrm{N} 1-\mathrm{Cu} 1-\mathrm{N} 3 & 116.7(3)^{\circ} \\ \mathrm{Cu} 1-\mathrm{N} 3 & 1.93(1) \AA & \angle \mathrm{N} 2-\mathrm{Cu} 1-\mathrm{N} 3 & 148.0(3)^{\circ} \\ \mathrm{N} 3-\mathrm{N} 4 & 1.47(2) \AA & \angle \mathrm{Cu} 1-\mathrm{N} 3-\mathrm{N} 4 & 117.2(8)^{\circ}\end{array}$

Table S7. Pertinent bond metrics of ( $\left.{ }^{\mathrm{EMind}} \mathrm{L}\right) \mathrm{CuI}(\mathbf{6})$.

$\begin{array}{cccc}\mathrm{Cu} 1-\mathrm{N} 1 & 1.913(6) \AA & \Sigma(\angle \mathrm{Cu} 1) & 360.1(3)^{\circ} \\ \mathrm{Cu} 1-\mathrm{N} 2 & 1.909(4) \AA & \angle \mathrm{N} 1-\mathrm{Cu} 1-\mathrm{I} 1 & 133.5(2)^{\circ} \\ \mathrm{Cu} 1-\mathrm{I} 1 & 2.399(1) \AA & \angle \mathrm{N} 2-\mathrm{Cu} 1-\mathrm{I} 1 & 130.7(1)^{\circ}\end{array}$




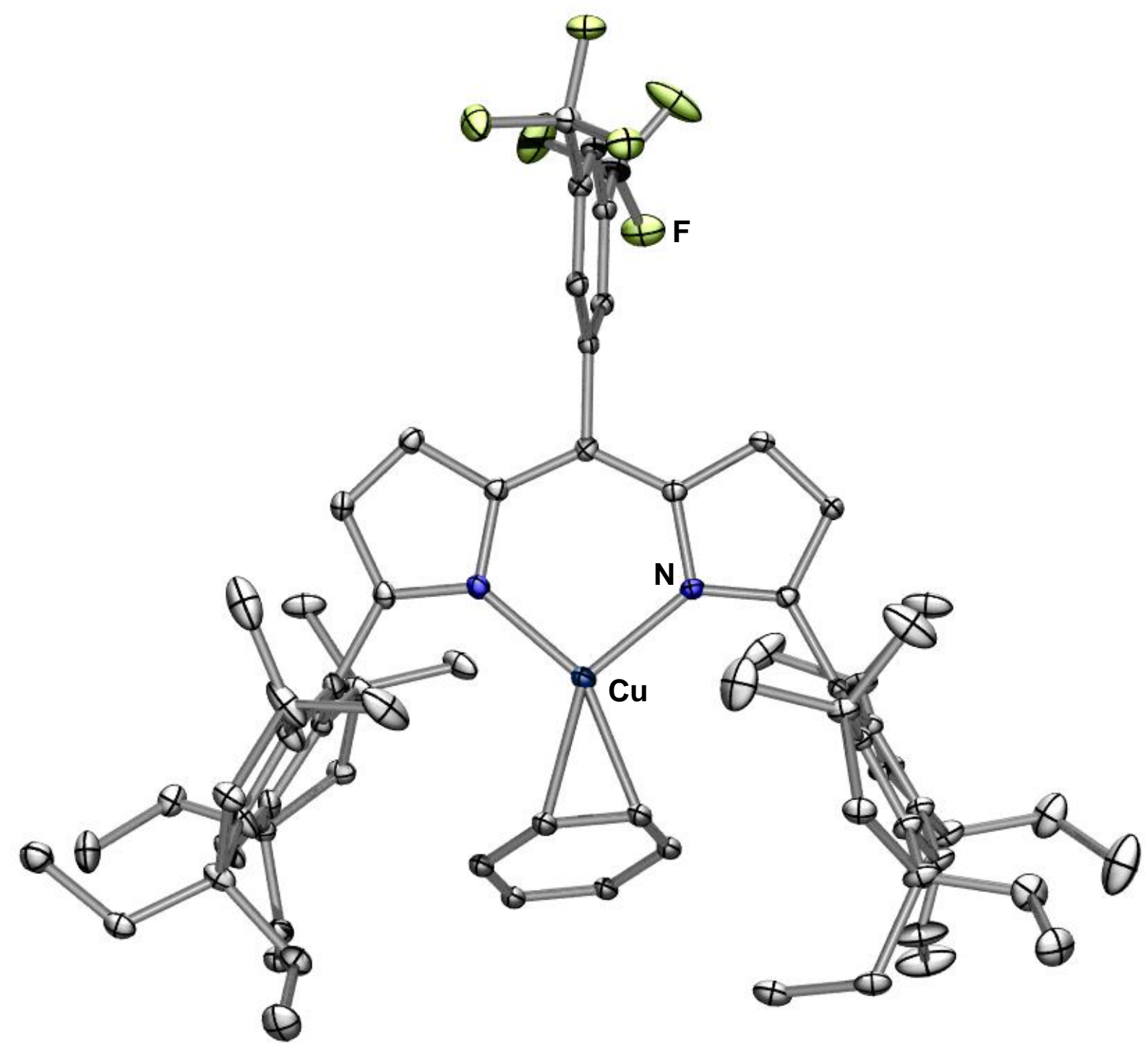

Figure S55. Solid-state molecular structure of $\left({ }^{E M i n d} L\right) C u\left(\eta^{2}-C_{6} H_{6}\right)$ with displacement ellipsoids at $50 \%$ probability level. Hydrogen atoms, structural disorder, and solvent molecules are omitted for clarity. Color scheme: $\mathrm{Cu}$ (cobalt blue), $\mathrm{F}$ (yellow-green), $\mathrm{N}$ (blue).

Single crystals suitable for X-ray diffraction were obtained through reverse vapor diffusion of hexamethyldisiloxane into a saturated pentane solution of 1 with excess benzene ( $c a .20$ equiv.) under Ar. In the presence of $\mathrm{N}_{2}$ and absence of excess benzene, reversion to $\left({ }^{\mathrm{EMind}} \mathrm{L}\right) \mathrm{Cu}\left(\mathrm{N}_{2}\right)$ is observed in both solution and solid state. 


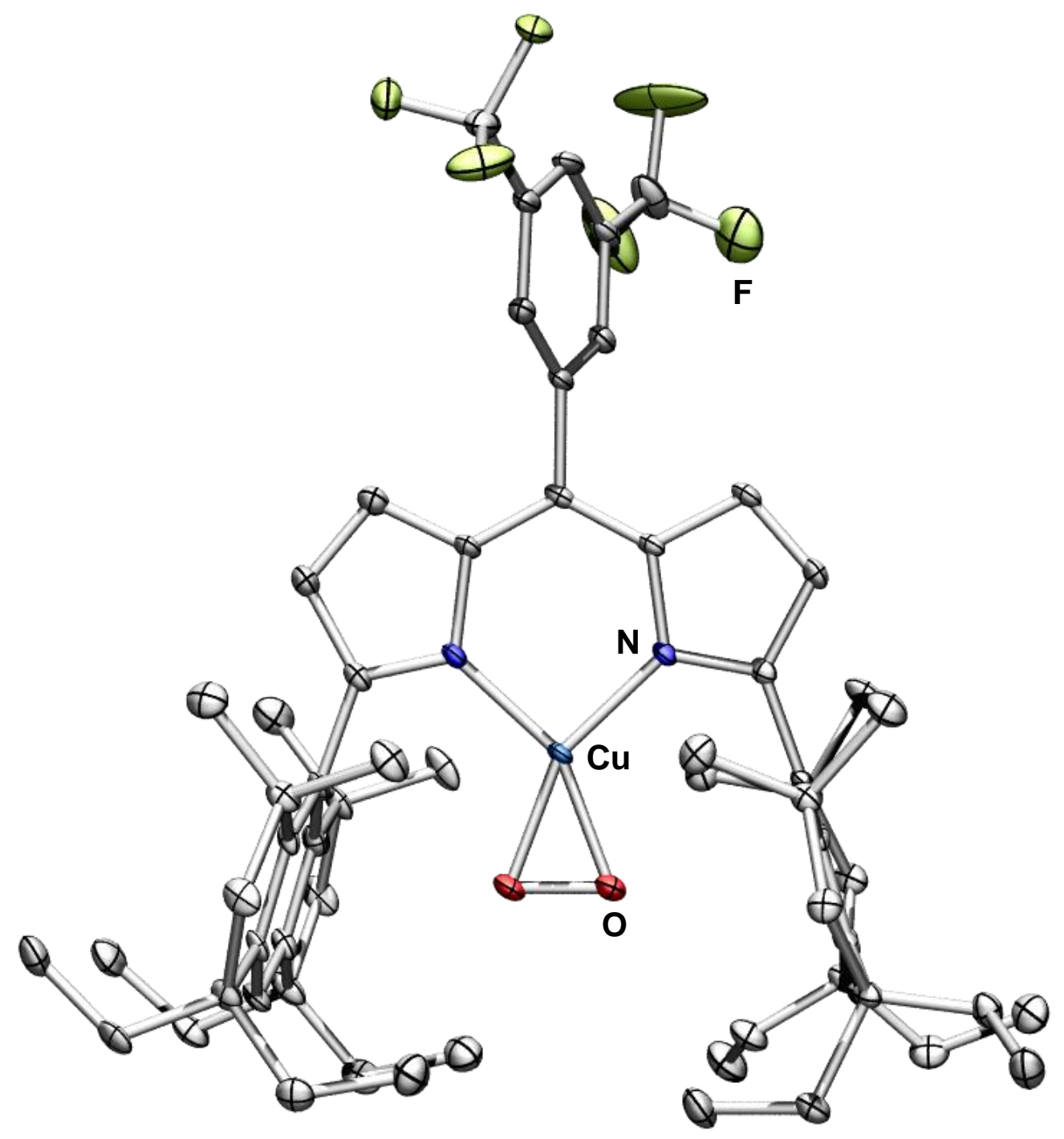

Figure S56. Solid-state molecular structure of ( $\left.{ }^{\mathrm{EMind}} \mathrm{L}\right) \mathrm{Cu}\left(\mathrm{O}_{2}\right)(2)$ with displacement ellipsoids at $50 \%$ probability level. Hydrogen atoms, structural disorder, and solvent molecules are omitted for clarity. Color scheme: $\mathrm{Cu}$ (cobalt blue), $\mathrm{F}$ (yellow-green), $\mathrm{N}$ (blue), $\mathrm{O}$ (red). 


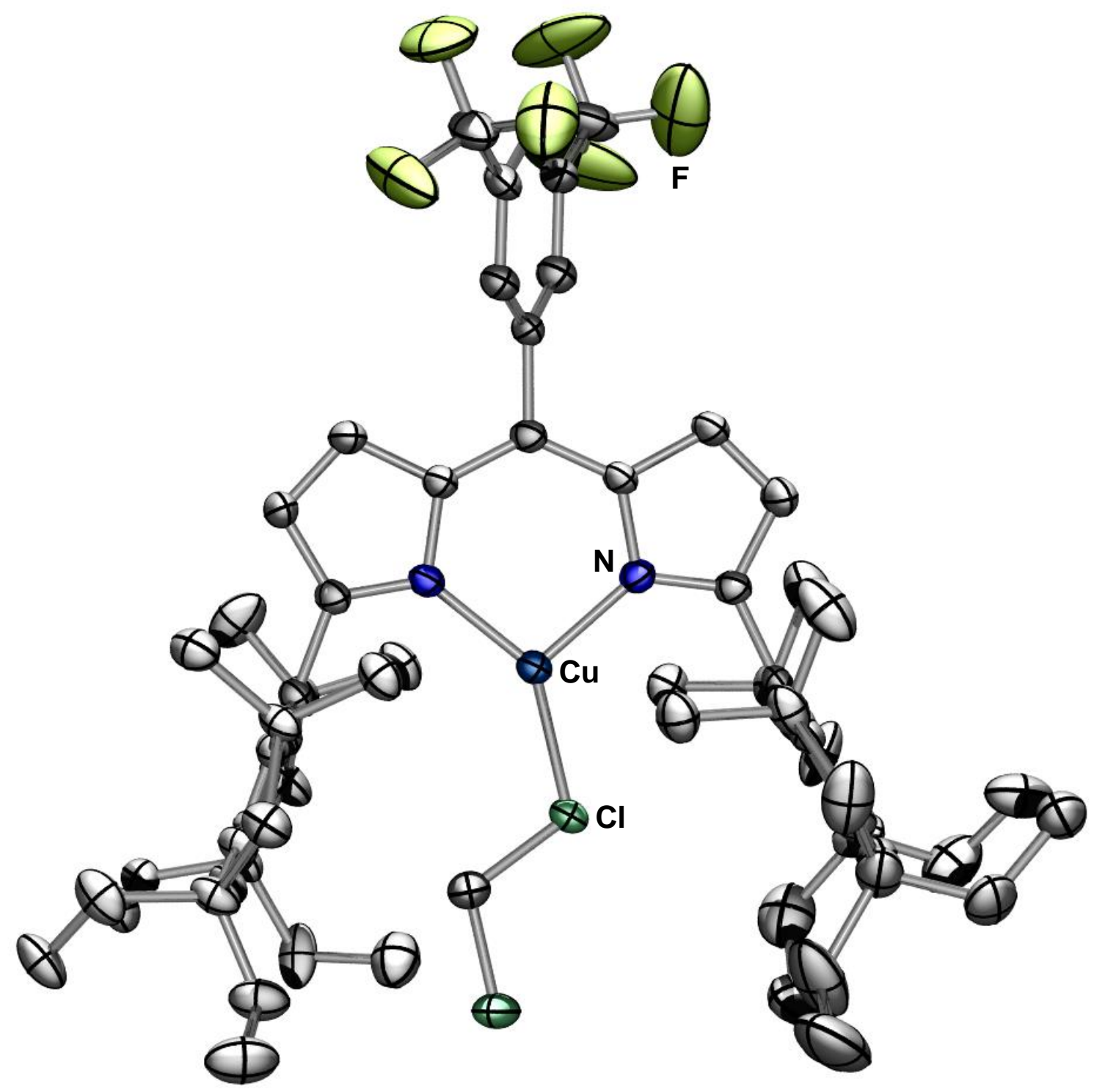

Figure S57. Solid-state molecular structure of ( $\left.{ }^{\mathrm{EMind}} \mathrm{L}\right) \mathrm{Cu}\left(\mathrm{CH}_{2} \mathrm{Cl}_{2}\right)$ (4) with displacement ellipsoids at $50 \%$ probability level. Hydrogen atoms, structural disorder, and solvent molecules are omitted for clarity. Color scheme: $\mathrm{Cu}$ (cobalt blue), $\mathrm{Cl}$ (green), $\mathrm{F}$ (yellow-green), $\mathrm{N}$ (blue). 


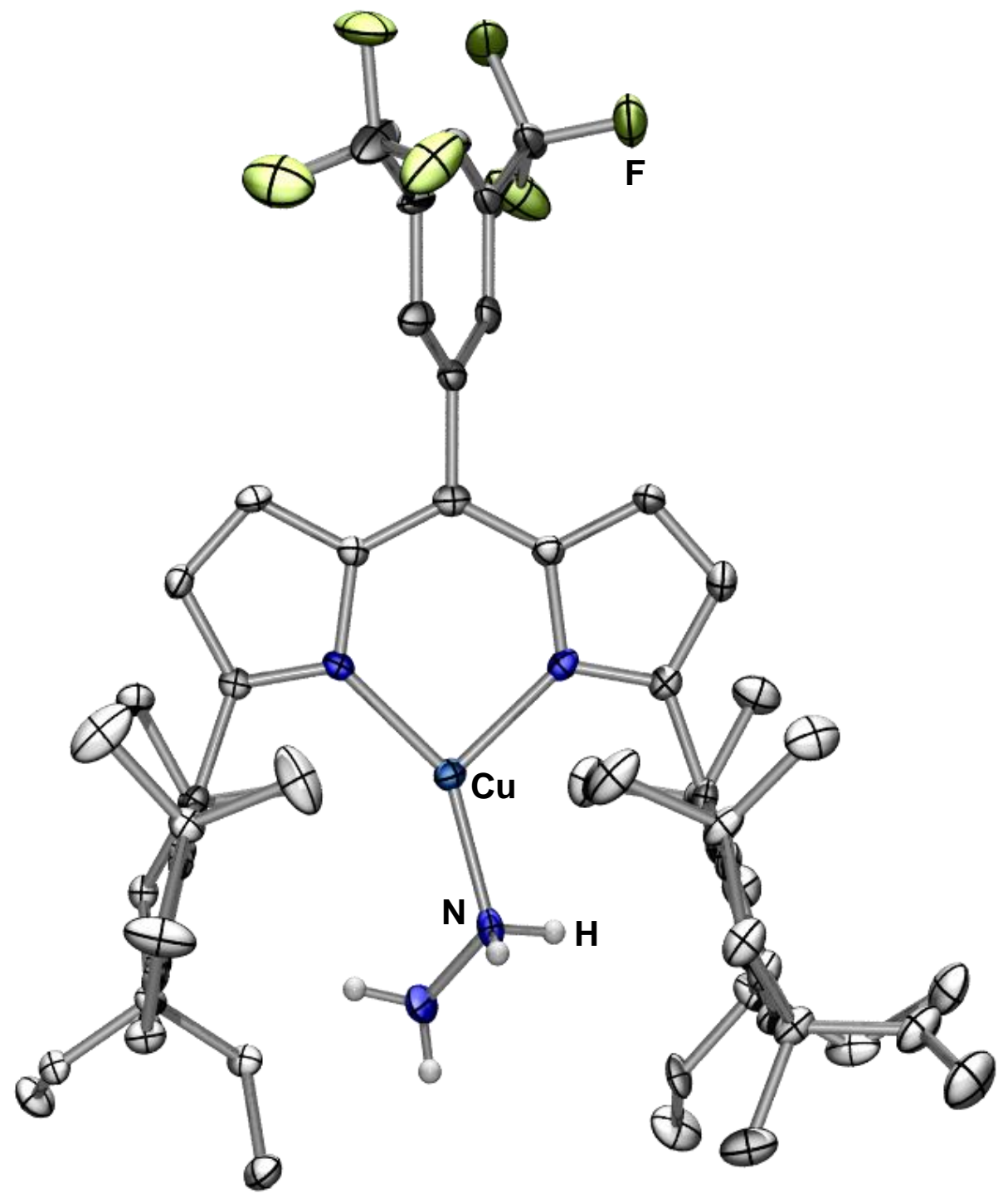

Figure S58. Solid-state molecular structure of ( $\left.{ }^{\mathrm{EMind}} \mathrm{L}\right) \mathrm{Cu}\left(\mathrm{NH}_{2} \mathrm{NH}_{2}\right)$ (5) with displacement ellipsoids at $50 \%$ probability level. Hydrogen atoms excluding those comprising the hydrazine fragment, structural disorder, and solvent molecules are omitted for clarity. Color scheme: $\mathrm{Cu}$ (cobalt blue), F (yellow-green), N (blue). 


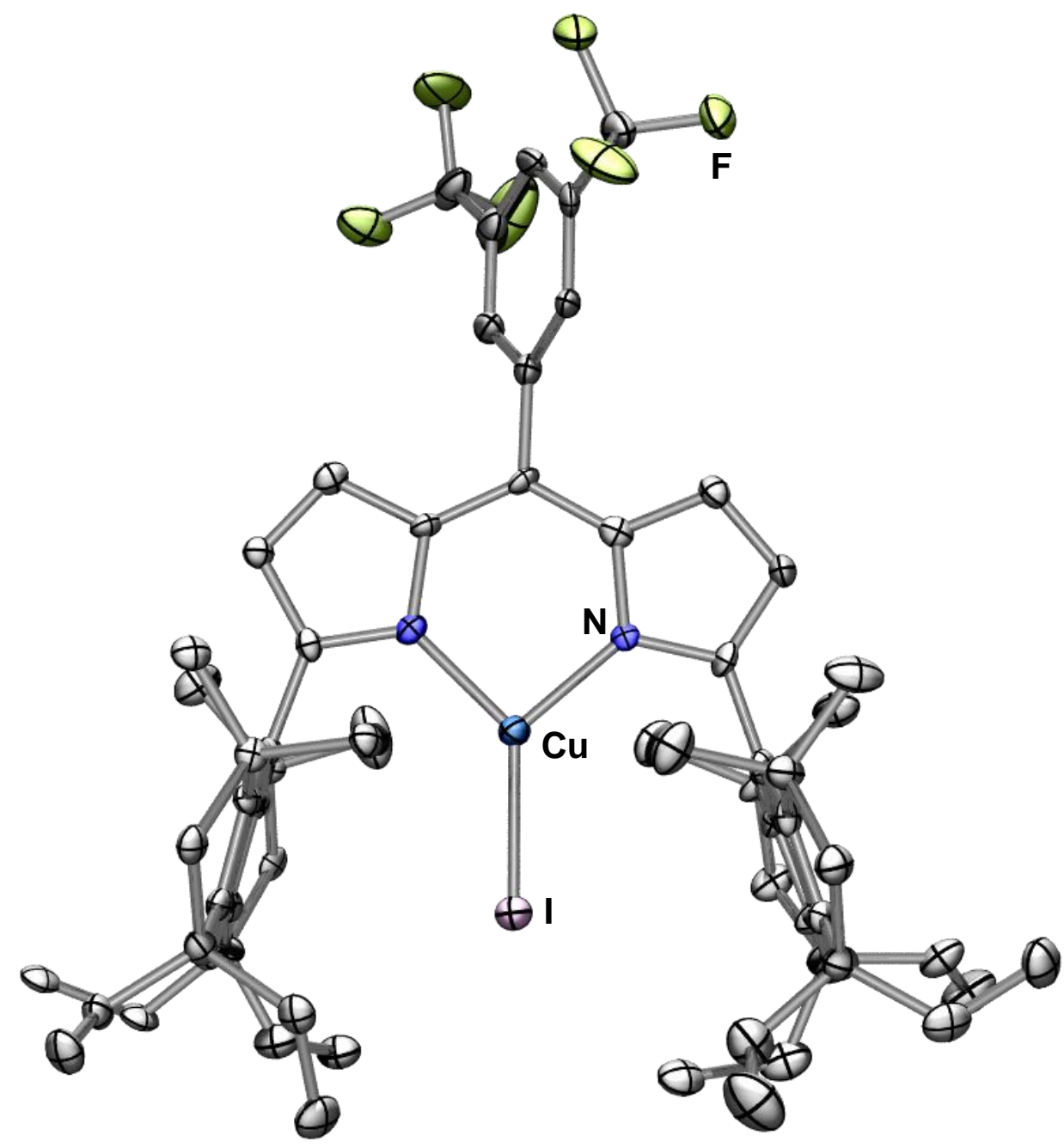

Figure S59. Solid-state molecular structure of $\left({ }^{\mathrm{EMind}} \mathrm{L}\right) \mathrm{CuI}(\mathbf{6})$ with displacement ellipsoids at 50 $\%$ probability level. Hydrogen atoms, structural disorder, and solvent molecules are omitted for clarity. Color scheme: $\mathrm{Cu}$ (cobalt blue), $\mathrm{F}$ (yellow-green), I (pink), $\mathrm{N}$ (blue). 


\section{References.}

(1) Weitz, I. S.; Rabinovitz, M., The Application of $\mathrm{C}_{8} \mathrm{~K}$ for Organic Synthesis: Reduction of Substituted Naphthalenes. J. Chem. Soc. Perkin 1 1993, 117.

(2) Kadassery, K. J.; Dey, S. K.; Cannella, A. F.; Surendhran, R.; Lacy, D. C., Photochemical Water-Splitting with Organomanganese Complexes. Inorg. Chem. 2017, 56, 9954.

(3) Konnick, M. M.; Guzei, I. A.; Stahl, S. S., Characterization of Peroxo and Hydroperoxo Intermediates in the Aerobic Oxidation of N-Heterocyclic-Carbene-Coordinated Palladium(0). $J$. Am. Chem. Soc. 2004, 126, 10212.

(4) Carsch, K. M.; DiMucci, I. M.; Iovan, D. A.; Li, A.; Zheng, S.-L.; Titus, C. J.; Lee, S. J.; Irwin, K. D.; Nordlund, D.; Lancaster, K. M.; Betley, T. A., Syntheses of a Copper-Supported Triplet Nitrene Complex Pertinent to Copper-Catalyzed Amination. Science 2019, 365, 1138.

(5) Stoll, S.; Schweiger, A., EasySpin, a Comprehensive Software Package for Spectral Simulation and Analysis in EPR. J. Magn. Reson. 2006, 178, 42.

(6) Francl, M. M.; Pietro, W. J.; Hehre, W. J.; Binkley, J. S.; Gordon, M. S.; DeFrees, D. J.; Pople, J. A., Self-Consistent Molecular Orbital Methods. XXIII. A Polarization-Type Basis Set for Second-Row Elements. J. Chem. Phys. 1982, 77, 3654.

(7) Hehre, W. J.; Ditchfield, R.; Pople, J. A., Self —Consistent Molecular Orbital Methods. XII. Further Extensions of Gaussian-Type Basis Sets for Use in Molecular Orbital Studies of Organic Molecules. J. Chem. Phys. 1972, 56, 2257.

(8) Grimme, S., Semiempirical Hybrid Density Functional with Perturbative Second-Order Correlation. J. Chem. Phys. 2006, 124, 034108.

(9) Becke, A. D., Density-Functional Thermochemistry. I. The Effect of the Exchange-Only Gradient Correction. J. Chem. Phys. 1992, 96, 2155.

(10) Barone, V.; Cossi, M., Quantum Calculation of Molecular Energies and Energy Gradients in Solution by a Conductor Solvent Model. J. Phys. Chem. A 1998, 102, 1995.

(11) Bochevarov, A. D.; Harder, E.; Hughes, T. F.; Greenwood, J. R.; Braden, D. A.; Philipp, D. M.; Rinaldo, D.; Halls, M. D.; Zhang, J.; Friesner, R. A., Jaguar: A High-Performance Quantum Chemistry Software Program with Strengths in Life and Materials Sciences. Int. J. Quantum Chem. 2013, 113, 2110.

(12) Busing, W. R.; Levy, H. A., Crystal and Molecular Structure of Hydrogen Peroxide: A Neutron-Diffraction Study. J. Chem. Phys. 1965, 42, 3054.

(13) Bruker AXS APEX3, Bruker AXS, Madison, Wisconsin, 2015.

(14) Krause, L.; Herbst-Irmer, R.; Sheldrick, G. M.; Stalke, D., Comparison of Silver and Molybdenum Microfocus X-Ray Sources for Single-Crystal Structure Determination. J. Appl. Crystallogr. 2015, 48, 3.

(15) Sheldrick, G., SHELXT - Integrated Space-Group and Crystal-Structure Determination. Acta Crystallogr. A 2015, 71, 3.

(16) Sheldrick, G., Crystal Structure Refinement with Shelxl. Acta Crystallogr. C 2015, 71, 3.

(17) Dolomanov, O. V.; Bourhis, L. J.; Gildea, R. J.; Howard, J. A. K.; Puschmann, H., Olex2: A Complete Structure Solution, Refinement and Analysis Program. J. Appl. Crystallogr. 2009, 42, 339.

(18) Spek, A., Single-Crystal Structure Validation with the Program PLATON. J. Appl. Crystallogr. 2003, 36, 7.

(19) Ramadhar, T. R.; Zheng, S.-L.; Chen, Y.-S.; Clardy, J., Analysis of Rapidly Synthesized Guest-Filled Porous Complexes with Synchrotron Radiation: Practical Guidelines for the Crystalline Sponge Method. Acta Crystallogr. A 2015, 71, 46. 
(20) Persistence of Vision Raytracer. http://www.povray.org/. 